\title{
1. CLAY MINERALOGY AND CLAY GEOCHEMISTRY IN THE VICINITY OF THE DÉCOLLEMENT ZONE, NORTHERN BARBADOS RIDGE ${ }^{1}$
}

\author{
Michael B. Underwood ${ }^{2}$ and Xinhua Deng ${ }^{2}$
}

\begin{abstract}
Fine-grained sediments recovered during Leg 156 of the Ocean Drilling Program contain assemblages of clay minerals that change considerably with depth. Key structural and stratigraphic features within the toe of the northern Barbados accretionary prism include the basal décollement and a sharp boundary between lithologic Units II and III; this lithologic boundary occurs within the décollement zone. We analyzed three size fractions $(4-8 \mu \mathrm{m}, 1-4 \mu \mathrm{m}$, and $<1 \mu \mathrm{m})$ by X-ray diffraction and obtained chemical data from the $<1-\mu \mathrm{m}$ size fractions by scanning electron microscopy coupled with energy dispersive spectroscopy. Relative proportions of expandable clay minerals increase significantly, but erratically, near the top of the décollement zone. Proportions of discrete smectite and smectite-rich illite/smectite (I/S) mixed-layer clays decrease steadily down the décollement zone. The base of the décollement zone is characterized by unusually high concentrations of illite. These changes in clay mineralogy influence the shear strength of claystones at the deformation front and help control the position of the décollement.

Relative abundances of illite within all lithofacies units increase in coarser size fractions, and this trend is consistent with a detrital source. Kaolinite does not display any systematic partitioning within Unit II, but its proportions increase in the finer size fractions within Unit III. The smectite content of Unit II increases with decreasing grain size; Unit III shows the opposite trend between particle size and \% smectite. Bentonite-like samples probably originated through in situ alteration of disseminated volcanic ash, but we believe that the pyroclastic material was mixed into a background sediment that already contained abundant detrital smectite. Illitic mixed-layer clays within the underthrust domain (Unit III) also appear to be detrital in origin. Correlations among both \% smectite and \% kaolinite and most major-element oxides are statistically significant, but correlations for illite are not. Expandable clays vary considerably in their chemical make-up; the most common type appears to be a $\mathrm{Mg}$-poor, K-rich, Fe-Al-beidellite. We rely upon actualistic provenance connections for late Quaternary clays to guide our interpretations of Miocene and Oligocene sediment dispersal. If these analogues are valid, then the proto-Orinoco and protoAmazon Rivers were the most important detrital sources for the older hemipelagic and turbidite deposits of Barbados Ridge.
\end{abstract}

\section{INTRODUCTION}

\section{Thematic Background}

Barbados Ridge (Fig. 1) is one of the best studied examples of intraoceanic subduction and frontal accretion (Westbrook, 1982; BijuDuval et al., 1982; Moore et al., 1982, 1988; Moore, Mascle, et al., 1990; Stride et al., 1982; Westbrook et al., 1988; Lallemant et al., 1990). The basal décollement of the Barbados accretionary prism has been imaged in unprecedented detail by a three-dimensional array of multichannel seismic-reflection profiles (Shipley et al., 1994; G.F. Moore et al., 1995), and the décollement zone was cored previously during Deep Sea Drilling Project (DSDP) Leg 78A (Biju-Duval, Moore, et al., 1984) and Ocean Drilling Program (ODP) Leg 110 (Mascle, Moore, et al., 1988). The primary thematic objectives of ODP Leg 156 have been to characterize, in detail, the geologic and geophysical properties of the décollement zone and to measure and monitor the physical and chemical effects of fluid flow within the décollement zone (Shipley, Ogawa, Blum, et al., 1995). One of the most important geologic parameters to consider in this regard is clay mineralogy, especially the abundance of smectite. Hydrated phyllosilicate minerals within the smectite group are very weak in terms of their mechanical properties, so one might expect to find faults propagating through smectite-rich stratigraphic intervals (Vrolijk, 1990).

Smectite also plays a critical role in the overall fluid budget of a subduction zone (Moore and Vrolijk, 1992). At low temperatures, in-

'Shipley, T.H., Ogawa, Y., Blum, P., and Bahr, J.M. (Eds.), 1997. Proc. ODP, Sci. Results, 156: College Station, TX (Ocean Drilling Program).

${ }^{2}$ Department of Geological Sciences, University of Missouri, Columbia, MO 65211, U.S.A. geoscmbu@showme.missouri.edu. terstitial-water molecules are transferred to interlayer sites in clay minerals as volcanic ash and volcanic-rock fragments are altered to smectite. At higher temperatures, diagenetic transformation of smectite to illite releases water, and this can lead to overpressured conditions within a stratal succession (Burst, 1969; Bruce, 1984; ColtenBradley, 1987; Freed and Peacor, 1989a). The smectite dehydration reaction usually begins at temperatures of about $60^{\circ} \mathrm{C}$ (Perry and Hower, 1970; Hower et al., 1976; Freed and Peacor, 1989b). Illitization appears to be gradual and progressive, however, and the intermediate products of the transformation consist of illite-smectite (I/S) mixed-layer clays (Dunoyer de Segonzac, 1970; Reynolds and Hower, 1970; Ahn and Peacor, 1986). I/S mixed-layer crystallites normally reach proportions of $70 \%-80 \%$ illite at temperatures of $90^{\circ}-140^{\circ} \mathrm{C}$ (Hower et al., 1976; Freed and Peacor, 1989b), but the socalled completion temperature (100\% illite) can extend beyond $200^{\circ} \mathrm{C}$ if kinetic factors are appropriate (Eberl and Hower, 1976; Hoffman and Hower, 1979; Bethke and Altaner, 1986; Velde and Vasseur, 1992; Huang et al., 1993).

Low-chlorinity pore waters have been documented near the toe of the Barbados accretionary prism, especially in the vicinity of the basal décollement (Gieskes et al., 1990b; Shipboard Scientific Party, 1995a, 1995b). Previous investigators suggested that these pore waters evolved during smectite-to-illite reactions within deeper domains of underplating and then migrated toward the prism's toe (Gieskes et al., 1990a; Wilkens et al., 1990; Vrolijk et al., 1991). Accurate numerical modeling of the entire prism-wide fluid budget depends on accurate documentation of clay mineral abundances within lithostratigraphic reference sections near the prism toe. It is likewise important to make accurate predictions of how those mineral assemblages will change with depth, structural position, temperature, and time. Knowledge of thermal structure, as well as the velocities of sed- 

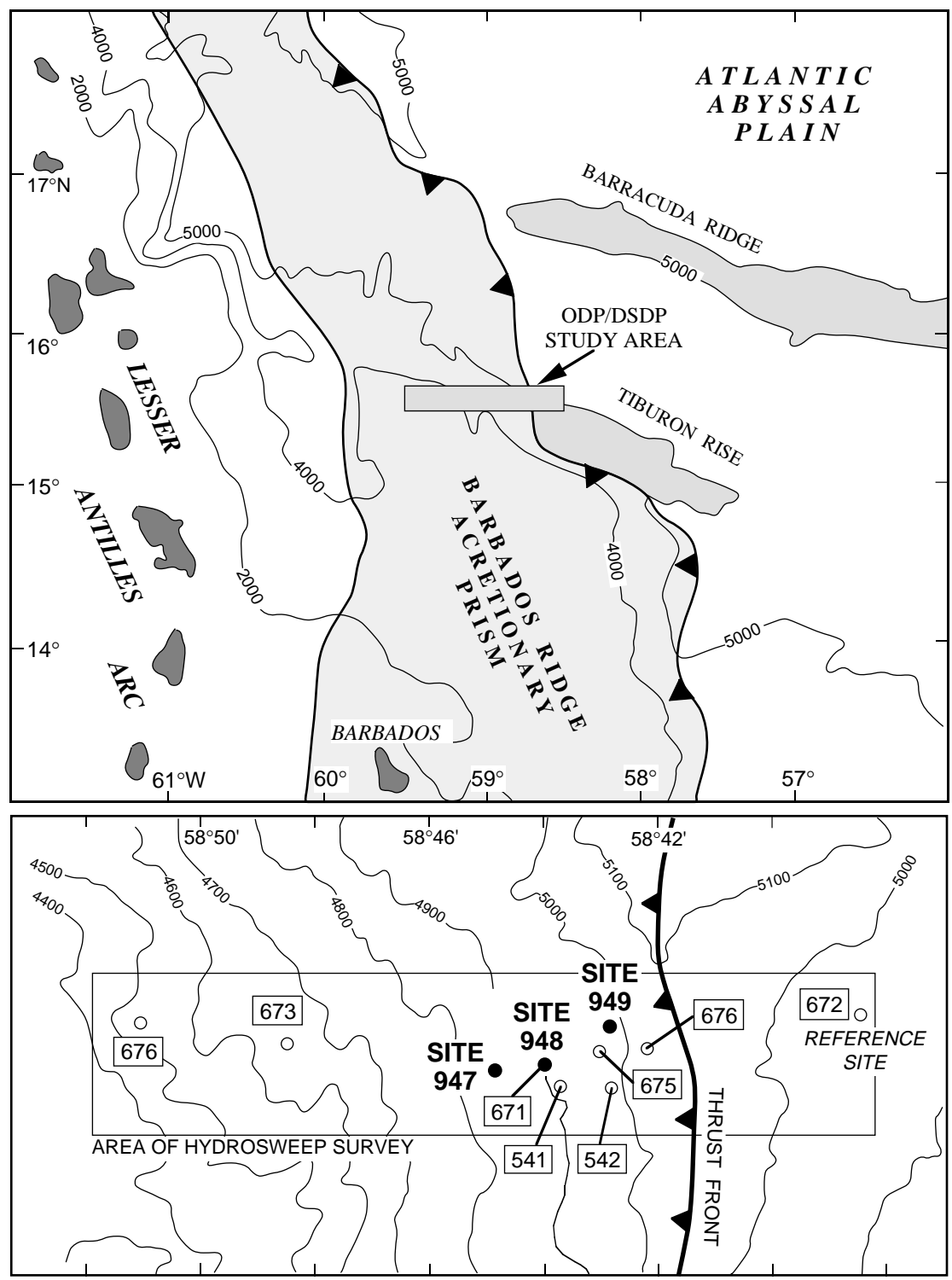

Figure 1. Location of study area. Enlarged bathymetric map (contours in meters) shows positions of drilling sites occupied during DSDP Leg 78A, ODP Leg 110, and Leg 156. iment movement within zones of frontal offscraping and underplating, present challenges of their own (Bekins and Dreiss, 1992; Ferguson et al., 1993). To complicate matters further, smectite-to-illite diagenesis may be occurring in situ near the Barbados décollement, but at unusually low temperatures (Tribble, 1990; Buatier et al., 1992; Tribble and Yeh, 1994). Acceleration of this important reaction may have occurred in response to the advection of pore water, but other workers have concluded that the I/S mixed-layer clays in question are detrital (Capet et al., 1990). This controversy certainly deserves additional attention. The spatial distribution of pressure-temperature windows of illitization must be incorporated into numerical models of prism-wide fluid budgets (Bekins et al., 1994), and because the mechanical properties of sediments also change along illitization pathways, this processs probably influences the behavior of the seismogenic zone that accompanies the plate boundary at depth (Kagami, 1985; Vrolijk, 1990).

Core recovery during Leg 156 was diminutive by typical ODP standards, but our sample density is unusually concentrated. During our study, clay minerals were analyzed both from discrete intervals of the cores and from trimmings of whole-round samples. This comprehensive approach has allowed us to define mineralogic gradients in exceptional detail in the vicinity of the décollement. The purposes of this paper are: (1) to document mineralogic variations as a function of grain size; (2) to document mineralogic variations as a function of stratigraphic position and structural domain; (3) to provide basic information regarding the character and abundance of illite-smectite mixed layer clays; and (4) to correlate clay mineralogy and geochemistry as indicators of detrital provenance and diagenesis.

\section{Geologic Background}

\section{Stratigraphy and Structure}

Geophysical data show that the Barbados accretionary prism narrows considerably with distance from the continental margin of South America (Fig. 1), probably in response to northward reductions in sediment influx and volume of offscraped sediments (Westbrook, 1982; Westbrook et al., 1984). A complicated array of Quaternary depositional systems characterizes the southern portion of the subduction zone (Faugères et al., 1991, 1993; Massé et al., 1995). Submarine canyons emanate from the Venezuelan shelf edge and cut obliquely across the accretionary prism to feed the braided channellevee system of the Orinoco Fan (Belderson et al., 1984). Turbidites 
also are ponded within intraslope basins. Abyssal-plain turbidites decrease in thickness, abundance, and grain size toward the north (Wright, 1984), where remobilization by contour currents becomes more common (Damuth, 1977).

The tectonostratigraphy of the northern Barbados Ridge was established by previous ODP and DSDP legs. Two reference sites were drilled outboard of the deformation front. Site 672 is located just north of the crest of Tiburon Rise (Fig. 1), where the stratigraphic succession consists of three fundamental units: (1) lower Pleistocene to Pliocene calcareous clay, mud, and marl, with abundant ash layers; (2) upper to middle Miocene mudstone and calcareous mudstone with local ash layers, and lower Miocene radiolarian-bearing mudstone; and (3) Eocene-Oligocene interbeds of claystone, calcareous claystone, chalk turbidites, terrigenous-silt turbidites, and quartzsand turbidites. The turbidite section at Site 672 is approximately 220 $m$ thick. The quartzose sands were eroded from South America and transported across the Atlantic Abyssal Plain; some were deposited at bathymetric positions that were elevated at least $800 \mathrm{~m}$ above the abyssal floor (Dolan et al., 1990). Site 543 is located approximately $20 \mathrm{~km}$ to the north of Site 672; the middle Eocene to Oligocene section is considerably thinner $(65 \mathrm{~m})$ there and completely devoid of turbidites (Biju-Duval, Moore, et al., 1984). Evidently, Site 543 was sheltered from turbidite influx during the Paleogene by the Tiburon Rise; Eocene-Oligocene deposits consist of Mn-stained radiolarian clay, zeolitic claystone, and claystone. At the base of the section, Maastrichtian to Campanian calcareous claystones overlie pillow basalts of the igneous basement.

The structural domain of frontal offscraping (Fig. 2) is characterized by thrust faults and numerous duplications of the lithostratigraphy (Moore et al., 1982, 1988). Correlations between acoustic facies and cores indicate that the contact between the lower Miocene interval of radiolarian claystone and the upper Oligocene interval of finegrained turbidites is a fundamental one. The basal décollement of the accretionary prism, for example, appears to propagate through this facies change at all of the sites cored to date (Moore et al., 1982, 1988). A prominent seismic reflector also can be traced well to the east of the deformation front (Fig. 2), and cores from one of the oceanic reference sites (Site 672) contain deformation features within lower Miocene claystones that are indicative of an incipient décollement (Shipboard Scientific Party, 1988b). Within the prism toe (Sites $541,671,675$, and 676), the décollement zone begins at a stratigraphic position close to or within the radiolarian mudstone, although there is some ambiguity in defining the exact top of the zone at all of those sites. The décollement was penetrated completely only at Site 671, but the lower limit of the décollement zone, as well as the position of the lithologic boundary between turbidites and radiolarian claystone, could not be pin-pointed due to incomplete core recovery (Shipboard
Scientific Party, 1988a). Coring at Site 671 extended $\sim 190-200 \mathrm{~m}$ below the top of the décollement (Fig. 2) to a depth of 691 meters below seafloor (mbsf).

Site 948 is located very close to the geographic position of Site 671 (Fig. 1). Coring began at a depth of $424 \mathrm{mbsf}$ and extended to 592 mbsf (Fig. 3). Interbeds of Oligocene calcareous turbidites, silty terrigenous turbidites, and hemipelagic claystones (Subunit IIIb) grade up-section into banded, variegated, Miocene claystones (Subunit IIIa). Six subunits were defined within the Miocene mudstones and claystones of Unit II (Shipboard Scientific Party, 1995a). The fundamental lithologic boundary between Units II and III occurs at a depth of 513.87 mbsf, within the lower Miocene sediments (Fig. 3). Based on occurrences of scaly fabric, the top of the décollement zone was placed at $498.10 \mathrm{mbsf}$; this position coincides with the uppermost recovery of Subunit IIe (radiolarian claystone), but other data indicate that the décollement's true upper limit may be slightly higher. Shipboard analyses of pore-water geochemistry, for example, showed that the minimum value of chloride occurs at a depth of $494.2 \mathrm{mbsf}$ (Core 156-948C-9X), and concentrations of methane are anomalously high at approximately the same position (Shipboard Scientific Party, 1995a). An abrupt change in strain geometry also was detected at a depth of $490 \mathrm{mbsf}$, based on analyses of magnetic fabric (Housen et al., 1996). This sharp decoupling horizon seems to be the most reliable way to define the position of the décollement. The lower limit of the décollement zone is at $529.80 \mathrm{mbsf}$, based on the last appearance of scaly fabric (Shipboard Scientific Party, 1995a).

Core recovery at Site 949 (Fig. 2) was patchy, but several key elements of the tectonostratigraphy were recognized (Shipboard Scientific Party, 1995b). Stratigraphic duplication due to a major thrust displacement occurs somewhere within a depth interval of 292.4$302.1 \mathrm{mbsf}$ (Fig. 3); this fault places fossil-barren claystone of Subunit IIc over nannofossil-rich claystone of Subunit IIb. The lithologic boundary between Units II and III was recovered at a depth of 430.92 mbsf. The upper and lower boundaries of the décollement zone, however, remain inferential. Operational boundaries were placed at 398.8 mbsf (first radiolarian claystone of Subunit IIe) and $437.1 \mathrm{mbsf}$ (bottom of Core 156-949B-22X), but the true boundaries almost certainly are located within intervals of no recovery.

\section{Clay Mineralogy}

Several attempts have been made to document relative abundances of clay minerals within the northern Barbados Ridge, as well as the two oceanic reference sites, but inconsistencies in laboratory methods hamper direct comparisons among the data sets. In addition, details regarding possible depth-dependent gradients in clay mineralogy, particularly as they might relate to development of the décolle-

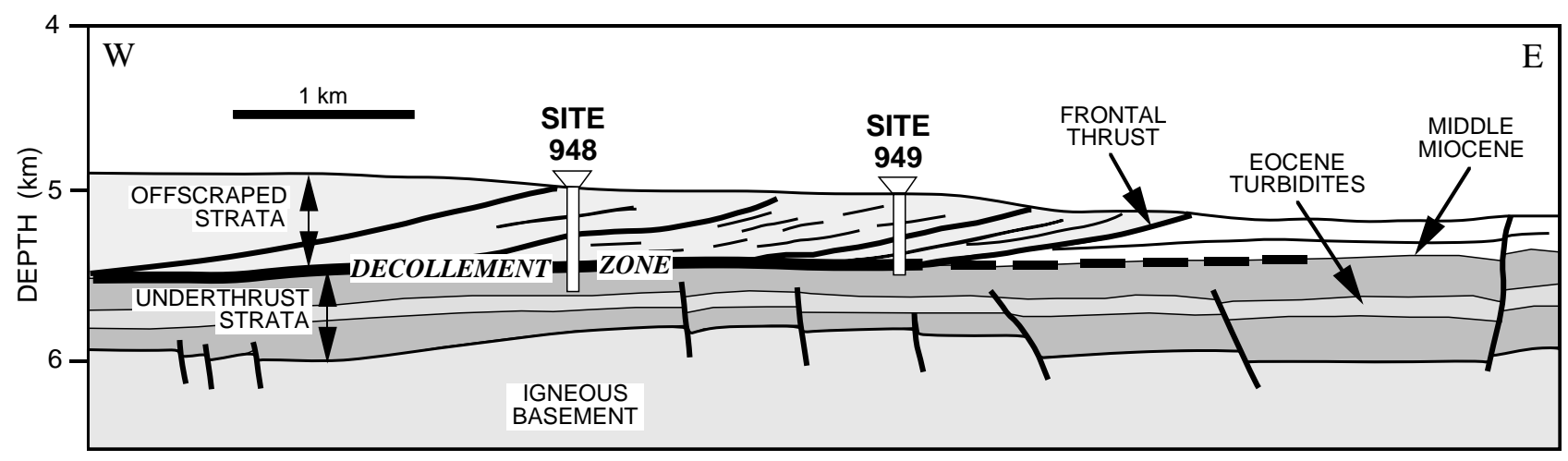

Figure 2. Interpretation of seismic reflection profile showing location of Site 948 with respect to major structural features. Site 949 is projected to its approximate position within the plane of this section. Modified from Moore et al. (1988). 


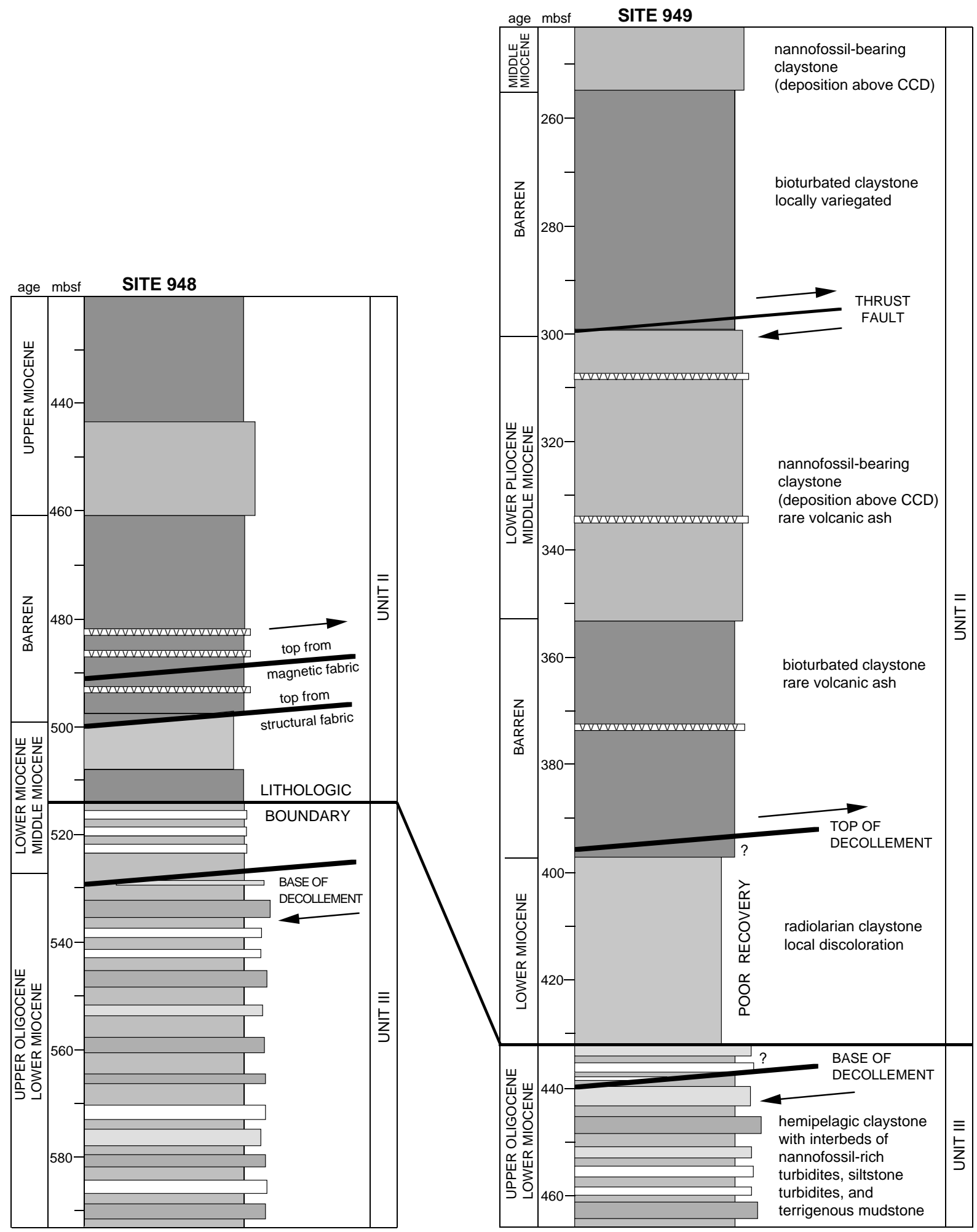

Figure 3. Interpretive stratigraphic columns and lithofacies correlations for Sites 948 and 949 . Upper boundary of the décollement zone at Site 948 varies depending on whether structural-fabric or magnetic-fabric criteria are used. Lithofacies and structural boundaries at Site 949 are uncertain due to poor core recovery. 
ment zone, were poorly defined by previous workers because their samples were widely spaced. Within stratigraphic intervals equivalent to those cored during Leg 156, Latouche and Maillet (1984) and Pudsey (1984) showed that the most prevalent clay minerals are smectite, kaolinite, and illite; chlorite, if present, occurs in minor to trace amounts. At Site 541, both of the DSDP Leg 78A studies showed that smectite content increases near the top of the décollement, reaching relative percentages greater than $80 \%$. Stratigraphic equivalents at Site 543 (i.e., lower Miocene radiolarian claystones) show similar trends, but the results of Pudsey (1984) also indicate that concentrations of illite reach unusually high values within upper Oligocene intervals that are correlative with strata at the base of the décollement zone. Pudsey (1984) considered the well-crystallized smectite to be authigenic in origin and called attention to its effect on bulk density, water content, shear strength, amount of deformation, and style of deformation. Latouche and Maillet (1984) attributed most of the temporal changes in clay mineralogy to differences in the balance between continental flux from South America (richer in illite and chlorite) and volcanogenic input from the Lesser Antilles arc (richer in smectite and kaolinite). Additional studies by Schoonmaker (1986) showed that most of the smectite-rich sediments contain a mixed-layer phase; samples adjacent to thrust faults and the décollement zone, however, contain nearly pure smectite (i.e., without mixed-layering). The expandable clay was identified erroneously as saponite, which is an unusual Mg-rich trioctahedral variety; this identification was subsequently retracted (Tribble, 1990).

Data obtained from ODP Leg 110 samples generally are consistent with those of previous studies. Capet et al. (1990) and Tribble (1990) both showed that smectite contents at Site 672 increase to $85 \%-90 \%$ near the top of the interval that corresponds to the incipient décollement. The anomalously course-grained succession of Eocene turbidites is similarly enriched in smectite $(60 \%-90 \%)$. At Site 671, lower Miocene radiolarian claystones near the top of the décollement zone are smectite rich. Capet et al. (1990) showed that the expandable clay is a variety of dioctahedral montmorillonite; they uncovered no evidence of clay diagenesis and regarded all of the temporal variations in clay mineralogy as responses to fluctuations in detrital influx from South America and the Lesser Antilles. In contrast, Tribble (1990) noted an apparent increase in the illite content of I/S mixed-layer phases at Site 671, beginning at the décollement zone, and attributed the acceleration of in situ diagenesis to effects of fluid flow.

Stimulated by these conflicting interpretations, Buatier et al. (1992) employed transmission and analytical electron microscopy to make direct observations of submicron-sized clay minerals from Site 671 . The most common smectite component is a very homogeneous, $\mathrm{K}$-rich variety, and the smectite is accompanied by large detrital crystals of illite and kaolinite, as well as minor amounts of chlorite and mixed-layer illite-chlorite. The two deepest samples (from 600 and $665 \mathrm{mbsf}$ ) also contain another phyllosilicate phase imbedded in a matrix of smectite; these crystallites resemble "neoformed" illite in the early stages of transition from smectite to I/S. Buatier et al. (1992) inferred that the illitization reaction is occurring in place in response to enhanced fluid flow, but they did not describe any samples from the décollement zone, itself. Their qualitative X-ray diffraction (XRD) results also indicate that the deeper samples with "neoformed" crystallites contain $\sim 50 \%$ expandable interlayers, whereas shallower samples typically contain more than $80 \%$ expandable interlayers. Buatier et al. (1992) did not calculate the volumetric abundance of the "neoformed" illite crystallites within the bulk clay-sized fraction. Consequently, the quantitative connection between their TEM image interpretations and XRD counts attributable to the "neoformed" illite remains somewhat unclear.

Tribble and Yeh (1994) completed analyses of oxygen isotope compositions for nine representative samples that had been collected from above, within, and below the décollement zone, as well as two
Oligocene samples from the oceanic reference site. The smectite and/or I/S phases were isolated by centrifugation from the bulk clay fraction, but the mineralogic purities and particle sizes of these extracts were not reported. All of the "pure-smectite" samples were obtained from intervals within or immediately above the décollement, and according to Tribble and Yeh (1994), those clays probably formed authigenically, either near the seafloor or during early burial. The two shallowest samples (with intermediate I/S proportions) are either products of weathering on volcanic islands of the Lesser Antilles or mixtures of authigenic and detrital phases. Data from the remaining samples below the décollement are similarly ambiguous. Tribble and Yeh (1994) favored an interpretation in which I/S clays in the underthrust domain (and stratigraphic equivalents at the oceanic reference site) formed by in situ diagenesis, but they also stated that erosion from a terrigenous source that had been exposed to deep burial dia-genesis "cannot be ruled out for the illite-rich illite-smectite on the basis of oxygen isotopic data alone."

\section{Geothermal Regime}

One of the difficulties in assessing the feasibility of in situ diagenesis of clay minerals, within or below the décollement zone, relates to the complexity of the geothermal regime. The geothermal character of the underthrust domain remains unexplored. Evidence from boreholes and near-surface temperature probes indicates that background values of heat flow near the toe of the Barbados accretionary prism are 35\%-40\% higher than expected for a simple conductive system involving oceanic lithosphere of Cretaceous age; heat transfer, therefore, must be influenced significantly by transient fluid flow within high-permeability pathways, particularly the décollement zone and related thrust faults that characterize the domain of frontal offscraping (Foucher et al., 1990; Fisher and Hounslow, 1990; Langseth et al., 1990).

Accurate calculations of the average geothermal gradient for Site 671 were hampered by insufficient data and unreliable measurements below 105 mbsf (Shipboard Scientific Party, 1988a). Based on those data, Tribble (1990) and Buatier et al. (1992) inferred that temperatures within the décollement zone are less than $25^{\circ} \mathrm{C}$, which is considerably less than conventional estimates for the onset of illitization (Freed and Peacor, 1989b). By combining data from Sites 671 and 948 , however, it was possible to define a new profile with two linear segments: $92^{\circ}-97^{\circ} \mathrm{C} / \mathrm{km}$ above $102.4 \mathrm{mbsf}$, and $66^{\circ} \mathrm{C} / \mathrm{km}$ between 247.1 and 420.5 mbsf (Shipboard Scientific Party, 1995a). If the deeper geothermal gradient continues to depths of 490-530 mbsf, then in situ temperatures within the décollement zone are actually $38^{\circ}-41^{\circ} \mathrm{C}$. The average geothermal gradient at Site 949 is well constrained at $80^{\circ} \mathrm{C} / \mathrm{km}$, so in situ temperatures within that portion of the décollement zone are approximately $34^{\circ}-38^{\circ} \mathrm{C}$ (Shipboard Scientific Party, 1995b). This result is almost identical to the average gradient calculated by Fisher and Hounslow (1990) for Site $672\left(79^{\circ} \mathrm{C} / \mathrm{km}\right)$, which means that temperature estimates for the proto-décollement zone at Site 672 are $16^{\circ}-19^{\circ} \mathrm{C}$.

\section{LABORATORY METHODS}

\section{$\mathrm{X}$-ray Diffraction}

Most samples were treated initially with dilute $(3 \%)$ hydrogen peroxide and distilled/deionized water to remove organic matter, disaggregated with an ultrasonic cell disrupter, and washed at least twice by centrifuge to remove salt. Residues of $<4-\mu \mathrm{m}$ material from grainsize analyses (FIS code) were exposed to higher concentrations of hydrogen peroxide and a hexametaphosphate dispersant (see Meyer and Fisher, Chapter 27, this volume, for additional details). We washed these specimens thoroughly before making the split at $1 \mu \mathrm{m}$, but differences in the initial treatment may have affected the XRD results. 
Splits of the 4- to $8-\mu \mathrm{m}, 1$ - to $4-\mu \mathrm{m}$, and $<1-\mu \mathrm{m}$ size fractions were isolated using a calibrated centrifuge at the following settings: $8 \mu \mathrm{m}$ $=200 \mathrm{rpm}$ for $3.3 \mathrm{~min} ; 4 \mu \mathrm{m}=300 \mathrm{rpm}$ for $5.9 \mathrm{~min} ; 1 \mu \mathrm{m}=1000 \mathrm{rpm}$ for $8.8 \mathrm{~min}$. We made a size split at $1 \mu \mathrm{m}$ (rather than $2 \mu \mathrm{m}$ ) because finer grained aggregates form smoother surfaces for improved analyses of clay chemistry by scanning electron microscopy coupled with energy dispersive spectroscopy. Oriented aggregates were prepared by vacuum-filtration and filter-peel transfer. Specimens were saturated with ethylene glycol by vapor exposure at $50^{\circ} \mathrm{C}$ for at least $24 \mathrm{hr}$ prior to X-ray analysis.

Clay minerals were analyzed using a Scintag PAD V XRD system interfaced with a Microvax 2000 microprocessor. Scanning parameters for routine analyses were set as follows: range $=2^{\circ}-20^{\circ} 2 \theta$; rate $=1^{\circ} 2 \theta / \mathrm{min}$; voltage $=40 \mathrm{kV}$ and $30 \mathrm{~mA}$; radiation $=\mathrm{Cu}_{\mathrm{Ka}}$; scan $=$ fast $/$ continuous; chopper increment (step) $=0.01^{\circ}$; and spinner $=$ off. Digital output was processed through a background correction and a deconvolution program designed to fit peaks to a Split Pearson VII profile shape (Gaussian-Lorentzian hybrid). Computer calculations of peak position $\left({ }^{\circ} 2 \theta\right)$, d-value $(\AA)$, intensity (counts/min), and integrated peak area (total counts) were made after processing by the deconvolution subroutine. Relative weight percentages of the dominant clay minerals are based on peak areas of the smectite (001), illite (001), and the composite chlorite $(002)+$ kaolinite $(001)$ reflections (Fig. 4). These three peaks have d-values of approximately $16.9 \AA$, $10.1 \AA$, and $7.1 \AA$, respectively. Because of potential interference between the chlorite (001) peak (14.2 $\AA$ ) and broad peaks produced by smectite and I/S mixed-layer phases, representative samples were heated to $550^{\circ} \mathrm{C}$ for $1 \mathrm{hr}$ and analyzed a second time. Heating causes dehydroxylation of the chlorite hydroxide sheet and changes its diffraction pattern; the diffraction pattern of kaolinite also disappears at this temperature. The amount of chlorite turned out to be below the level of detection in all samples tested.

Most data sets that describe clay-mineral abundances for marine sediments are based on the Biscaye (1965) weighting factors (McManus, 1991). These factors are 1X for smectite, 2X for kaolinite, and $4 \mathrm{X}$ for illite. Tests of accuracy (using measured weight percentages of mineral standards from the Clay Minerals Society Repository) showed that the error associated with this method can be as high as 20\% (Underwood et al., 1993a). To improve accuracy, correction factors need to be adjusted as the absolute abundances of each clay mineral change and in response to variations in illite crystallinity, smectite chemistry, and peak broadening caused by I/S. Work is in progress to derive new weighting factors based on mineral standards extracted from the Barbados sediments. We did not attempt to estimate the absolute percentages of I/S mixed-layer clay with respect to the total clay-mineral population, and variable amounts of mixed-layer crystallites probably have been recorded in the counts used to estimate both discrete \% illite and \% smectite. Our data, therefore, should be viewed as semi-quantitative relative abundances.

Internal precision of XRD data depends on inherent inhomogeneities in mineralogy, sample preparation, stability of the diffractometer, and the software used to process digital output and deconvolute peaks. To test each of these, we prepared ten different slides of a single sample, analyzed one slide ten times, and processed one set of digital data ten times. Standard deviations about mean values of $\%$ smectite, $\%$ illite, and $\%$ kaolinite are as follows: sample-preparation test: smectite $=3.3 \%$, illite $=2.1 \%$, kaolinite $=1.8 \%$; hardware-stability test: smectite $=3.7 \%$, illite $=2.6 \%$, kaolinite $=2.3 \%$; software test: smectite $=0.6 \%$, illite $=0.7 \%$, kaolinite $=0.8 \%$.

Several methods exist to calculate the ratios of illite and smectite crystallites in mixed-layer phases (e.g., Reynolds and Hower, 1970; Weir et al., 1975; Srodon, 1980, 1981; Tomita et al., 1988; Inoue et al., 1989). As shown in Figure 4, we calculated the angular separation $\left(\Delta^{\circ} 2 \theta\right)$ between the composite illite-(001)/smectite-(002) reflection (at $\sim 9^{\circ}$ to $10^{\circ} 2 \theta$ ) and the composite illite-(002)/ smectite-(003) reflection (at $\sim 16^{\circ}$ to $17^{\circ} 2 \theta$ ) and utilized a third-order polynomial equation calculated from the data summarized by Moore and
Reynolds (1989). This method is more reliable than those that use the position of a single peak, because it minimizes errors caused by sample misalignment and inconsistent glycol-layer thickness. Hathon (1992) showed that I/S proportions determined in this way are between $2 \%$ and $8 \%$ of values extracted from visual counts of layer thickness on transmission electron microscopy (TEM) images. Some of the peak intensities generated by the composite $\mathrm{I}(001) / \mathrm{S}(002)$ and $\mathrm{I}(002) / \mathrm{S}(003)$ peaks are quite subdued (Fig. 4); peak positions were considered unreliable unless they could be fit to an ideal peak shape above the background. Samples representing a range of I/S compositions also were analyzed under three different conditions (air-dried, glycol-saturated, and heated to $375^{\circ} \mathrm{C}$ ) to make sure that the peaks in question conformed to the expected behavior of mixed-layer I/S, as summarized by Moore and Reynolds (1989). A second set of calculations was made using the saddle/001 peak intensity ratio, as described by Inoue et al. (1989). These values were obtained from the same sets of digital data but without processing by the backgroundcorrection program. As emphasized by Inoue et al. (1989), the accuracy of this method depends on the crystallite size distribution, the degree of preferred ordering of crystallites, and the abundance of discrete illite. Errors are probably greater than $15 \%$. Finally, for Site 948 , we completed additional analyses of the $<0.2-\mu \mathrm{m}$ size fraction to help verify depth-dependent trends.

Recognition of the degree of ordering in interstratified I/S requires careful analysis of the low-angle region of diffractograms. Random stacking of interlayers in the I/S phase $(\mathrm{R}=0)$ and/or nearly pure (discrete) smectite will produce peaks near $5^{\circ} 2 \theta(\mathrm{d}$-value $=16.9$ $\AA$ ). This peak increases in intensity and shifts closer to $6.5^{\circ} 2 \theta$ (dvalue $=13.3 \AA)$ if a $1: 1$ stacking arrangement $(R=1)$ becomes dominant, and second-order reflections $(002 *)$ of the $27-\AA$ superstructure are generated. We did not detect any ordering of the $(\mathrm{R}=1)$ type.

\section{SEM/EDS Geochemistry}

Semi-quantitative chemical analyses of the $<1-\mu \mathrm{m}$ size fraction were carried out by scanning electron microscopy coupled with energy dispersive spectroscopy (SEM/EDS), following the procedure of Ross et al. (1993). The same oriented aggregates of clay-sized particles were used for both XRD and SEM/EDS. All slides were coated with $20 \mathrm{~nm}$ of carbon for conductivity. An Amray 1600T SEM was set at $15 \mathrm{KeV}$ and a $0.7 \mathrm{nA}$ beam current. The Kevex EDS system contains a standard Be window detector and a 4PI multichannel analyzer; analyses were completed using a $30^{\circ}$ take-off angle and a collection time of $120 \mathrm{~s}$ per spot. The size of the raster area (at $1000 \mathrm{X}$ magnification) is $51,750 \mu \mathrm{m}^{2}$, so each set of counts represents a bulk analysis of a composite mineral mixture that contains many overlapping crystals.

A well-characterized standard specimen of illite (IMt-1) was used to calibrate EDS data for $\mathrm{Al}_{2} \mathrm{O}_{3}, \mathrm{SiO}_{2}, \mathrm{~K}_{2} \mathrm{O}$, and $\mathrm{Fe}$-oxides (reported as $\mathrm{Fe}_{\text {total }}$ ). Standard smectite (SHca-1) was used for $\mathrm{MgO}$ and $\mathrm{CaO}$, and a rutile standard was used to calibrate $\mathrm{TiO}_{2}$. Concentrations of $\mathrm{Na}$ and $\mathrm{Mn}$ were not determined. Each of the homogeneous mineral standards had been analyzed quantitatively for all major oxides by X-ray fluorescence (XRF) prior to our study, and those values were used to characterize the standard compositions. System software consists of the DeskTop Spectrum Analyzer from the National Institute of Standards and Technology and the ZAF correction procedure, which converts EDS counts to elemental weight fractions. Our elemental weight fractions are average values based on measurements of two raster areas per sample, and conversions of those data to oxide weight percents were made using standard gravimetric factors. We analyzed five samples a second time as a test of reproducibility. The average differences for each oxide are as follows: $\mathrm{SiO}_{2}=0.84 \mathrm{wt} \% ; \mathrm{Al}_{2} \mathrm{O}_{3}=$ $1.07 \mathrm{wt} \% ; \mathrm{Fe}_{\text {total }}=0.40 \mathrm{wt} \% ; \mathrm{K}_{2} \mathrm{O}=0.21 \mathrm{wt} \% ; \mathrm{MgO}=0.37 \mathrm{wt} \%$; $\mathrm{CaO}=0.44 \mathrm{wt} \%$; and $\mathrm{TiO}_{2}=0.36 \mathrm{wt} \%$. We emphasize here that the purpose of these analyses was to document relative trends in compositional variables, particularly as they pertain to changes in clay min- 

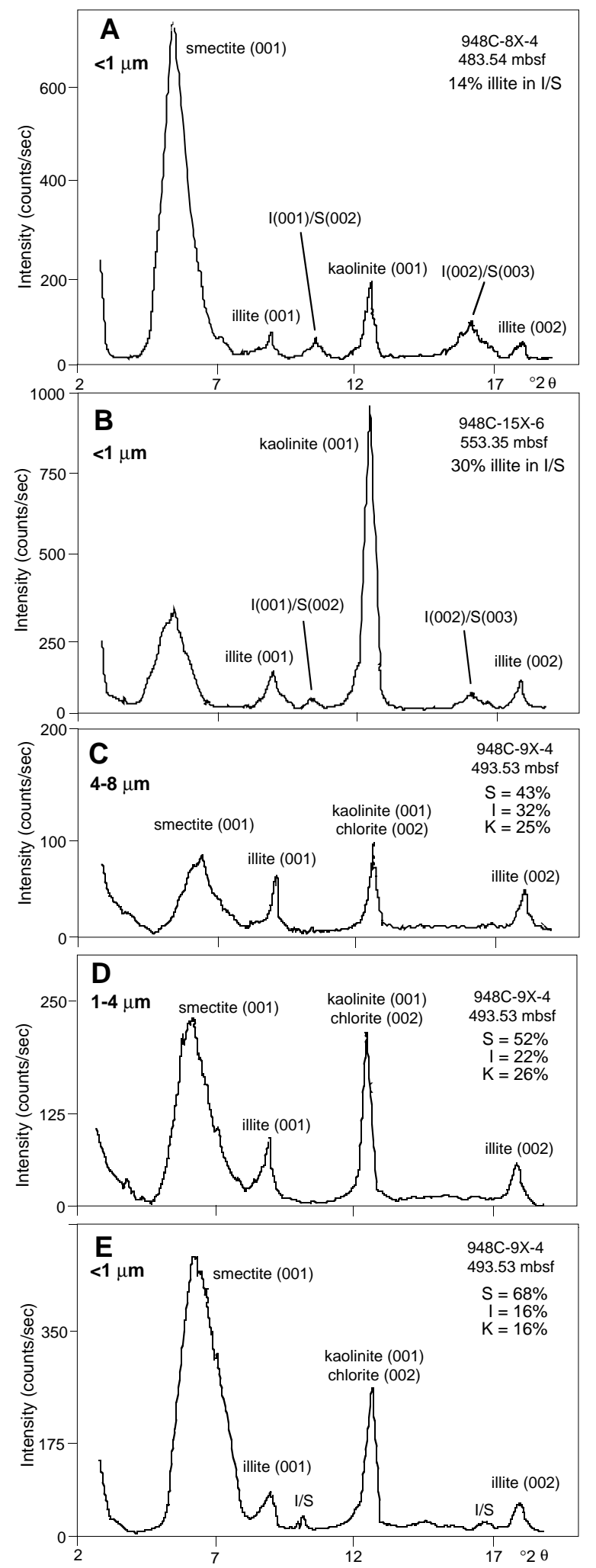

Figure 4. X-ray diffractograms for representative glycol-saturated oriented aggregates. Examples $\mathbf{A}$ and $\mathbf{B}$ illustrate the variable positions and intensities of basal reflections for smectite, illite, and kaolinite, together with composite peaks produced by illite/smectite mixed-layer clays. Examples $\mathbf{C}, \mathbf{D}$, and $\mathbf{E}$ illustrate differences among 4 - to $8-\mu \mathrm{m}, 1$ - to $4-\mu \mathrm{m}$, and $<1-\mu \mathrm{m}$ size fractions for a single sample. eralogy. The accuracy of absolute elemental concentrations remains questionable.

\section{RESULTS}

\section{Relative Abundances of Clay Minerals}

\section{Site 948}

Any interpretation of clay-mineral abundances should be placed within the context of each specimen's bulk mineralogy. Bulk-powder XRD analyses are notoriously inaccurate when expressed in terms of absolute mineral abundances, but we consider the shipboard results from Leg 156 to be better than most because peak-area weighting factors were adjusted using a program of matrix singular value decomposition (Fisher and Underwood, 1995). Figure 5 displays calculated weight percentages of total clay minerals (relative to quartz, feldspar, and calcite) for Site 948 (see Shipboard Scientific Party, 1995a, for data tables). Values throughout Unit II are quite uniform. The totalclay content decreases gradually from the top of Subunit IIa ( $68 \%)$ to the base of Subunit IId ( $54 \%)$, and then increases consistently from the top of Subunit IIe to the base of Subunit IIf $(\sim 72 \%)$. This finding is contrary to the data of Tribble (1990), which show a decrease in total-clay below the top of the décollement zone at Site 671. The content of total-clay below the lithologic boundary (within Unit III) fluctuates considerably (Fig. 5). This scatter exists because samples in Unit III were obtained from interbeds of pelagic claystone, nannofossil-rich lime-mud turbidites, and terrigenous-mud turbidites.

Table 1 contains results of XRD analysis of clay minerals from Site 948, including the average relative percentages for each lithostratigraphic unit. Figure 6 shows relative percentages of the three most abundant clay minerals within the $<1-\mu \mathrm{m}$ size fraction (smectite, illite, and kaolinite). Because of differences in sample type (discrete intervals vs. whole-round trimmings) and sample preparation (i.e., FIS grain-size residues), we also grouped data for individual clay minerals by sample code (Fig. 7). Except for one sample, smectite content within Subunits IIa and IIb is between $31 \%$ and $53 \%$. Smectite content increases in Subunits IIc and IId to averages of 56\% and $61 \%$, respectively. Lower Miocene radiolarian claystones of Subunit IIe, which seemingly form a marker interval near the top of the décollement zone, range from $32 \%$ to $71 \%$ smectite. Most of the highest values of $\%$ smectite $(70 \%-80 \%)$ occur within the upper part of the décollement zone or immediately above the top of the décollement zone. This noteworthy result is consistent with the previous findings from Site 541 (Pudsey, 1984) and Site 671 (Capet et al., 1990; Tribble, 1990). The added detail of our dataset, however, permits clear definition of a depth profile in which smectite values decrease progressively down the décollement zone. Subunits IIIa and IIIb contain averages of $22 \%$ and $20 \%$ smectite, respectively, and the lowest value detected in our study (3\%) occurs immediately below the structurally defined base of the décollement zone (Fig. 7).

Values of \% illite show trends opposite to those of smectite (Fig. 7). Illite contents decrease systematically from the top of Subunit IIa to the top of the décollement zone and then increase steadily down the décollement zone, reaching a maximum value of $50 \%$ at its structurally defined base. Percentages of illite display considerable scatter within Subunit IIIb. Trends for \% kaolinite are not as clear as those for illite and smectite, but most values immediately above the top of the décollement zone are between $15 \%$ and $25 \%$, and there is an obvious increase in kaolinite down the décollement zone (Fig. 7). Average values of $\%$ kaolinite for Subunits IIIa and IIIb are $42 \%$ and $51 \%$, respectively. Interbeds of pelagic claystone, calcareous turbidites, and terrigenous turbidites within Unit III vary considerably in their contents of kaolinite (Fig. 7).

One of the goals of our study was to determine whether or not systematic partitioning of clay minerals occurs as a function of grain 


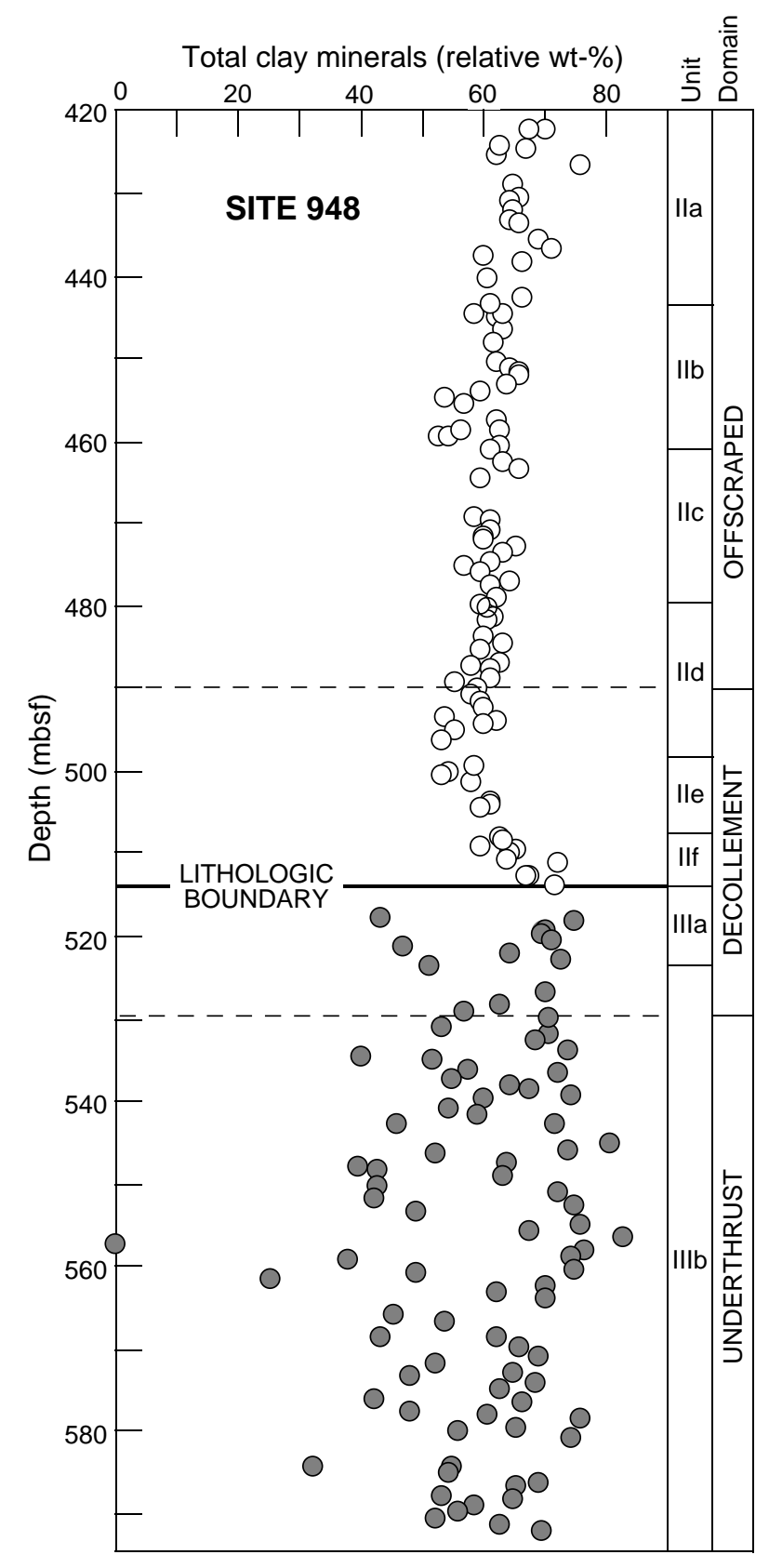

Figure 5. Relative abundance of total clay minerals in bulk samples from Site 948. See Shipboard Scientific Party (1995a) for additional data.

size. Within Unit II, relative percentages of smectite tend to increase systematically from coarser to finer size fractions, whereas relative percentages of illite increase with increasing grain size (Fig. 8). Kaolinite content, however, does not change much with particle size, and those changes are not systematic. Mineral partitioning is fundamentally different within Unit III (Fig. 8). Relative percentages of both smectite and illite generally increase with increasing size, and relative abundance of kaolinite increases with decreasing particle size.

\section{Site 949}

Figure 9 shows calculated weight percentages of total clay minerals (relative to quartz, feldspar, and calcite) for Site 949 (see Ship- board Scientific Party, 1995b, for data tables). Core recovery at this site was less than ideal, so trends in mineralogy cannot be defined in detail. Relative percentages of total clay minerals display more scatter, but results are consistent with bulk-powder data from comparable lithostratigraphic units at Site 948 (Fig. 5).

Table 2 lists results of XRD analyses of clay minerals from Site 949. Poor sample recovery precludes detailed definition of mineralogy gradients, but most of the results are consistent with findings from Site 948. Patterns of clay-mineral distribution within Unit II show increases in smectite and decreases in illite with decreasing particle size (Fig. 10). Clay minerals also change across major structural discontinuities. An inferred thrust fault at $\sim 298 \mathrm{mbsf}$ is based on repetition of Subunits IIb and IIc, and it is clear that the clay mineralogy also changes across this zone. Relative percentages of smectite within the $<1-\mu \mathrm{m}$ size fraction increase to $62 \%-80 \%$ above the fault and decrease to $18 \%-39 \%$ below the fault (Fig. 11). Percentages of illite are $12 \%-23 \%$ above the fault and $26 \%-41 \%$ below the fault. Kaolinite content likewise decreases above the fault $(7 \%-24 \%)$ and increases immediately below the fault $(25 \%-43 \%)$. The same type of relationship between clay mineralogy and the location of thrust faults within the offscraped domain was recognized by Tribble (1990) at Site 671. With respect to the décollement zone, smectite content within the $<1-\mu \mathrm{m}$ size fraction is high above its top and lowest below its base (Fig. 11). Percentages of illite and kaolinite do not vary dramatically within the décollement zone, but both reach maxima below its base. Precise definition of boundaries for the décollement zone is impossible, but if one superimposes the depth gradients in clay mineralogy from Site 948 through correlative intervals with no recovery at Site 949, then it appears as though the lower limit of the décollement zone occurs at a depth of $\sim 450$ mbsf (i.e., just above the top of Core 156-949B-25H), and the top is probably just above $400 \mathrm{mbsf}$.

\section{I/S Mixed-Layer Clays}

Table 3 presents data for the smectite (001) reflection, the $\mathrm{I}(001) /$ $\mathrm{S}(002)$ reflection, the $\mathrm{I}(002) / \mathrm{S}(003)$ peak, and the saddle/001 peak intensity ratio for smectite plus I/S. Relative proportions of illite and smectite within the I/S phases show clear trends at Sites 948 , but a more scatter pattern at Site 949 (Fig. 12). Most proportions of \% illite vary between $10 \%$ and $40 \%$ above the top of the décollement zone at Site 948 , regardless of the particle size or the method of calculation. The smectite-rich interval above the Unit II/III boundary (470-510 mbsf) displays a notable decrease in the proportion of illite interlayers, with values between $5 \%$ and $22 \%$. All of the smectite-rich clays within these specimens yielded d-values for the (001) reflection that are within the ranges expected for nearly pure smectite or smectiterich $\mathrm{I} / \mathrm{S}$ with random $(\mathrm{R}=0)$ ordering. In contrast, most samples from below the lithologic boundary contain between $22 \%$ and $48 \%$ illite. Data from Site 949 are more limited and fail to define clear differences across either the décollement zone or the lithologic boundary (Fig. 12).

\section{Clay Composition}

Hathon and Underwood (1991) demonstrated the effectiveness of SEM/EDS as a method for documenting chemical variations in assemblages of clay-sized minerals from near-surface sediments. One obvious limitation is that chemical signals from constituents other than phyllosilicates (e.g., calcareous nannofossils, volcanic ash, biogenic silica) can weaken correlations among relative percentages of specific clay minerals and weight-percentages of major oxides. In the case of the Barbados Ridge, Buatier et al. (1992) recognized detrital grains of quartz, plagioclase, and titanomagnetite, plus authigenic grains of K-feldspar and titanium oxide. Chemical variability within individual clay-mineral groups (especially smectite) is another factor to consider. Finally, it is virtually impossible to isolate the elemental contributions of individual mixed-layer crystallites, even by analyti- 
Table 1. X-ray diffraction data for three size fractions of clay minerals from mudstones and claystones at Site 948.

\begin{tabular}{|c|c|c|c|c|c|c|c|c|c|c|c|c|c|c|c|c|c|c|c|c|c|}
\hline \multirow{3}{*}{$\begin{array}{l}\text { Core, section, } \\
\text { interval }(\mathrm{cm})\end{array}$} & \multirow{3}{*}{$\begin{array}{l}\text { Depth } \\
\text { (mbsf) }\end{array}$} & \multirow{3}{*}{$\begin{array}{l}\text { Sample } \\
\text { code }\end{array}$} & \multirow{3}{*}{$\begin{array}{l}\text { Strat. } \\
\text { unit }\end{array}$} & \multicolumn{6}{|c|}{ 4- to $8-\mu \mathrm{m}$ size fraction } & \multicolumn{6}{|c|}{ 1-4- $\mu \mathrm{m}$ size fraction } & \multicolumn{6}{|c|}{$<1-\mu \mathrm{m}$ size fraction } \\
\hline & & & & \multicolumn{3}{|c|}{ Integrated peak area (counts) } & Estir & ted per & ntage & Integrate & peak are & $\mathrm{a}$ (counts) & Estir & ed per & ntage & Integratec & peak are & ea (counts) & Estir & ed per & ntage \\
\hline & & & & Smectite & Illite & Kaolinite & $\% \mathrm{~S}$ & $\% \mathrm{I}$ & $\% \mathrm{~K}$ & Smectite & Illite & Kaolinite & $\% \mathrm{~S}$ & $\% \mathrm{I}$ & $\% \mathrm{~K}$ & Smectite & Illite & Kaolinite & $\% \mathrm{~S}$ & $\% \mathrm{I}$ & $\% \mathrm{~K}$ \\
\hline 156-948B- & & & & & & & & & & & & & & & & & & & & & \\
\hline $1 \mathrm{H}-1,32-37$ & 0.32 & UND & I & 1,532 & 990 & 1,370 & 19 & 48 & 33 & 3,566 & 1,518 & 1,973 & 26 & 45 & 29 & 6,717 & 2,894 & 4,653 & 24 & 42 & 34 \\
\hline $1 \mathrm{H}-2,15-20$ & 1.65 & UND & I & 1,696 & 991 & 1,627 & 19 & 44 & 37 & 3,377 & 1,860 & 2,422 & 22 & 48 & 31 & 6,787 & 4,143 & 7,112 & 18 & 44 & 38 \\
\hline $1 \mathrm{H}-3,26-30$ & 3.26 & UND & I & 1,646 & 1,458 & 1,422 & 16 & 57 & 28 & 2,062 & 1,503 & 2,055 & 17 & 49 & 34 & 2,688 & 2,355 & 2,775 & 15 & 53 & 31 \\
\hline $1 \mathrm{H}-4,143-150$ & 5.93 & IW & I & & & & & & & & & & & & & 3,837 & 680 & 1,702 & 39 & 27 & 34 \\
\hline $\begin{array}{l}1 \mathrm{H}-5,105-110 \\
\text { Mean }\end{array}$ & 7.05 & UND & I & 3,088 & 1,523 & 2,120 & $\begin{array}{l}23 \\
19\end{array}$ & $\begin{array}{r}45 \\
49\end{array}$ & 32 & 3,438 & 1,252 & 1,775 & 29 & 42 & 30 & 3,468 & 1,647 & 2,369 & 23 & 45 & 32 \\
\hline $156-948 \mathrm{C}-$ & & & & & & & & & & & & & & & & & & & & & \\
\hline $2 \mathrm{X}-1,140-141$ & 422.20 & FIS & IIa & & & & & & & & & & & & & 11,748 & 3,099 & 5,725 & 33 & 35 & 32 \\
\hline $2 X-3,64-67$ & 424.44 & UND & IIa & 4,123 & 906 & 1,166 & 41 & 36 & 23 & 7,229 & 2,718 & 2,024 & 33 & 49 & 18 & 19,334 & 2,474 & 3,730 & 53 & 27 & 20 \\
\hline $2 \mathrm{X}-4,130-150$ & 426.60 & IW & IIa & & & & & & & & & & & & & 18,044 & 5,192 & 7,923 & 33 & 38 & 29 \\
\hline $2 \mathrm{X}-6,41-46$ & 428.71 & UND & IIa & 4,737 & 1,758 & 2,653 & 28 & 41 & 31 & 8,505 & 2,575 & 3,547 & 33 & 40 & 27 & 24,270 & 4,246 & 6,833 & 44 & 31 & 25 \\
\hline $3 \mathrm{X}-1,11-14$ & 430.61 & FIS & IIa & דונדוד & 1,150 & 2,000 & 20 & 41 & $J_{1}$ & 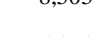 & 2, & , & ה & 40 & 21 & 17,565 & 2,549 & 5,253 & 46 & 27 & 27 \\
\hline $3 \mathrm{X}-2,20-24$ & 432.20 & UND & IIa & 3,979 & 1,623 & 2,536 & 26 & 42 & 33 & 5,191 & 1,927 & 2,392 & 29 & 44 & 27 & 50,907 & 2,449 & 6,187 & 70 & 13 & 17 \\
\hline $3 \mathrm{X}-3,102-106$ & 434.52 & UND & IIa & 3,310 & 1,620 & 2,626 & 22 & 43 & 35 & 7,729 & 2,162 & 3,176 & 34 & 38 & 28 & 33,494 & 3,697 & 9,132 & 50 & 22 & 27 \\
\hline $3 \mathrm{X}-5,120-150$ & 437.70 & IW & IIa & & & & & & & & & & & & & 10,903 & 2,411 & 6,841 & 32 & 28 & 40 \\
\hline $3 \mathrm{X}-6,88-92$ & 438.88 & UND & IIa & 4,341 & 1,345 & 1,874 & 32 & 40 & 28 & 7,126 & 2,151 & 3,479 & 31 & 38 & 31 & 21,642 & 3,737 & 11,668 & 36 & 25 & 39 \\
\hline $4 \mathrm{X}-2,30-41$ & 441.90 & JA & IIa & 5,992 & 1,278 & 2,284 & 38 & 33 & 29 & 6,907 & 1,812 & 3,047 & 34 & 36 & 30 & 14,996 & 2,644 & 5,680 & 41 & 29 & 31 \\
\hline $4 \mathrm{X}-2,114-117$ & 442.74 & UND & IIa & 2,205 & 890 & 1,487 & 25 & 41 & 34 & 5,090 & 1,347 & 3,654 & 29 & 30 & 41 & 30,612 & 5,561 & 16,442 & 36 & 26 & 38 \\
\hline $\begin{array}{l}\text { 4X-3, 58-61 } \\
\text { Mean }\end{array}$ & 443.68 & FIS & IIa & & & & 30 & 39 & 30 & & & & 32 & 39 & 29 & 32,770 & 4,494 & 16,770 & $\begin{array}{l}39 \\
43\end{array}$ & 21 & 40 \\
\hline $4 \mathrm{X}-4,55-58$ & 445.15 & UND & $\mathrm{IIb}$ & 4,510 & 1,152 & 1,763 & 36 & 36 & 28 & 4,912 & 1,522 & 2,937 & 29 & 36 & 35 & 18,348 & 3,510 & 13,168 & 31 & 24 & 45 \\
\hline $4 \mathrm{X}-5,110-150$ & 447.20 & IW & $\mathrm{IIb}$ & & & & & & & & & & & & & 16,698 & 2,443 & 2,180 & 54 & 32 & 14 \\
\hline $4 \mathrm{X}-6,49-52$ & 448.09 & UND & $\mathrm{IIb}$ & 5,685 & 1,047 & 1,755 & 42 & 31 & 26 & 8,177 & 1,269 & 1,988 & 47 & 29 & 23 & 34,819 & 3,773 & 8,357 & 52 & 23 & 25 \\
\hline $5 \mathrm{X}-1,142-146$ & 451.22 & FIS & $\mathrm{IIb}$ & & & & & & & , & 1,20 & 1,700 & (1) & 25 & 25 & 24,484 & 2,337 & 6,502 & 52 & 20 & 28 \\
\hline $5 \mathrm{X}-2,85-89$ & 452.15 & UND & $\mathrm{IIb}$ & 6,499 & 1,994 & 2,951 & 32 & 39 & 29 & 7,873 & 3,216 & 4,703 & 26 & 43 & 31 & 25,586 & 2,175 & 8,901 & 49 & 17 & 34 \\
\hline $5 \mathrm{X}-2,120-150$ & 452.50 & IW & $\mathrm{IIb}$ & & & & & & & & & & & & & 22,916 & 2,435 & 10,570 & 43 & 18 & 39 \\
\hline $5 \mathrm{X}-4,39-43$ & 454.69 & UND & $\mathrm{IIb}$ & 4,289 & 695 & 1,075 & 47 & 30 & 23 & 7,267 & 1,189 & 3,145 & 40 & 26 & 34 & 27,589 & 3,566 & 11,127 & 43 & 22 & 35 \\
\hline $5 \mathrm{X}-6,142-146$ & 458.72 & FIS & $\mathrm{IIb}$ & $+, 20\rangle$ & (1) & 1,071 & th & 50 & 25 & , & 1,107 & (14) & 40 & 20 & $J 7$ & 17,229 & 2,321 & 10,151 & 37 & 20 & 43 \\
\hline $\begin{array}{l}6 \mathrm{X}-1,115-119 \\
\text { Mean }\end{array}$ & 460.55 & UND & $\mathrm{IIb}$ & 5,056 & 1,458 & 1,673 & $\begin{array}{l}36 \\
38\end{array}$ & $\begin{array}{l}41 \\
36\end{array}$ & $\begin{array}{l}24 \\
26\end{array}$ & 5,835 & 1,560 & 4,123 & $\begin{array}{l}29 \\
34\end{array}$ & $\begin{array}{l}31 \\
33\end{array}$ & $\begin{array}{l}41 \\
33\end{array}$ & 20,920 & 1,704 & 8,508 & $\begin{array}{l}47 \\
45\end{array}$ & $\begin{array}{l}15 \\
21\end{array}$ & 38 \\
\hline $6 \mathrm{X}-2,57-60$ & 461.47 & UND & IIc & 7,637 & 1,775 & 4,185 & 33 & 31 & 36 & 5,664 & 2,165 & 5,520 & 22 & 34 & 44 & 27,854 & 4,441 & 27,194 & 28 & 18 & 54 \\
\hline & 463.16 & UND & IIc & 6,505 & 1,261 & 2,001 & 42 & 32 & 26 & 11,700 & 2,141 & 4,461 & 40 & 29 & 31 & 30,133 & 2,084 & 9,201 & 53 & 15 & 32 \\
\hline $6 \mathrm{X}-4,118-150$ & 465.08 & IW & IIc & & & 2,001 & 42 & & & 11,700 & $2,14+1$ & $4,+01$ & 40 & 27 & 正 & 32,154 & 1,977 & 3,846 & 67 & 17 & 16 \\
\hline $7 \mathrm{X}-1,7-10$ & 469.07 & FIS & IIc & & & & & & & & & & & & & 16,938 & 4,017 & $\begin{array}{l}7,040 \\
7,108\end{array}$ & 36 & 34 & $\begin{array}{l}10 \\
30\end{array}$ \\
\hline $7 \mathrm{X}-2,43-46$ & 470.93 & FIS & IIc & & & & & & & & & & & & & $\begin{array}{l}10,930 \\
21,663\end{array}$ & $\begin{array}{l}4,011 \\
2,334\end{array}$ & 6,250 & 50 & 21 & 29 \\
\hline $7 X-3,2-5$ & 472.02 & FIS & IIc & & & & & & & & & & & & & 18,902 & 1,780 & 4,748 & 53 & 20 & 27 \\
\hline $7 \mathrm{X}-4,115-118$ & 474.65 & UND & IIc & 7,549 & 1,694 & 2,742 & 38 & 34 & 28 & 6,726 & 1,706 & 2,900 & 35 & 35 & 30 & 38,348 & 1,628 & 4,723 & 71 & 12 & 17 \\
\hline $7 X-5,15-18$ & 475.15 & FIS & IIc & & & & & & & & & & & & & 25,468 & 2,815 & 6,329 & 52 & 23 & 26 \\
\hline $7 X-6,41-44$ & 476.91 & UND & IIc & 11,912 & 1,526 & 2,983 & 50 & 25 & 25 & 15,179 & 1,764 & 3,275 & 53 & 25 & 23 & 30,695 & 674 & 3,231 & 77 & 7 & 16 \\
\hline $7 \mathrm{X}-6,120-150$ & 477.70 & IW & IIc & & & & & & & & & & & & & 19,092 & 1,190 & 2,937 & 64 & 16 & 20 \\
\hline $8 X-1,48-52$ & 479.18 & FIS & IIc & & & & & & & & & & & & & 23,276 & 2,547 & 6,772 & 50 & 22 & 29 \\
\hline $8 X-1,56-58$ & 479.26 & UND & IIc & 6,324 & 1,000 & 1,171 & 50 & 32 & 18 & 7,806 & 1,629 & 1,190 & 47 & 39 & 14 & 30,832 & 1,477 & 3,711 & 70 & 13 & 17 \\
\hline Mean & & & & & & & 43 & 31 & 27 & & & & 39 & 32 & 28 & & & & 56 & 18 & 26 \\
\hline $8 \mathrm{X}-3,110-150$ & 482.80 & MK & IId & 5,948 & 1,378 & 2,402 & 37 & 34 & 30 & 10,880 & 2,371 & 4,654 & 37 & 32 & 31 & 26,939 & 1,687 & 5,935 & 59 & 15 & 26 \\
\hline $8 \mathrm{X}-4,34-36$ & 483.54 & UND & IId & 6,020 & 1,008 & 1,321 & 47 & 32 & 21 & 4,292 & 759 & 1,668 & 40 & 28 & 31 & 28,412 & 907 & 2,610 & 76 & 10 & 14 \\
\hline $8 \mathrm{X}-4,70-80$ & 483.90 & $\mathrm{JA}$ & IId & 3,410 & 927 & 1,087 & 37 & 40 & 23 & 4,148 & 1,106 & 1,335 & 37 & 39 & 24 & 24,438 & 1,201 & 2,901 & 70 & 14 & 17 \\
\hline $8 \mathrm{X}-4,141-148$ & 484.61 & FIS & IId & & & & & & & & & & & & & 20,8 & 1,522 & 2,3 & 66 & 1 & 15 \\
\hline $8 \mathrm{X}-6,64-66$ & 486.84 & FIS & IId & & & & & & & & & & & & & 31,343 & 2,784 & 6 & 56 & 20 & 24 \\
\hline $8 \mathrm{X}-6,120-150$ & 487.40 & IW & IId & & & & & & & & & & & & & 20 & 2,059 & 5 & 51 & 20 & 29 \\
\hline $8 X-7,6$ & & UND & IId & 5,180 & 1,210 & 1,5 & 39 & 37 & 24 & 8,0 & 1,99 & 3,8 & 34 & 34 & 33 & 29,8 & 1,987 & 5,330 & 62 & 16 & 22 \\
\hline $9 X-1,42-46$ & 488.82 & UND & IId & 6,135 & 1,867 & 1,8 & 36 & 43 & 21 & 11,025 & 2,294 & 4,4 & 38 & 32 & 30 & 28,779 & 1,621 & 4,843 & 64 & 14 & 22 \\
\hline $9 \mathrm{X}-2,26-30$ & 490.16 & UND & IId & 4,788 & 802 & 1,397 & 44 & 30 & 26 & 7,366 & 1,631 & 2,660 & 38 & 34 & 28 & 24,188 & 1,484 & 4,351 & 62 & 15 & 22 \\
\hline $9 \mathrm{X}-2,86-90$ & 490.76 & FIS & IId & & & & & & & & & & & & & 20,068 & 453 & 6,911 & 56 & 5 & 39 \\
\hline $9 \mathrm{X}-3,82-86$ & 492.22 & FIS & IId & & & & & & & & & & & & & 36,840 & 2,190 & 4,057 & 69 & 16 & 15 \\
\hline $9 X-4,63-67$ & 493.53 & UND & IId & 9,884 & 1,803 & 2,844 & 43 & 32 & 25 & 16,523 & 1,701 & 4,106 & 52 & 22 & 26 & 39,065 & 2,289 & 4,477 & 68 & 16 & 16 \\
\hline $9 \mathrm{X}-4,96-100$ & 493.86 & FIS & IId & , & 1,003 & 2,044 & 43 & 52 & 25 & 10,203 & 1,101 & 4,100 & $J 2$ & 22 & 20 & 28,489 & 3,536 & 10,872 & 44 & $\begin{array}{l}10 \\
22\end{array}$ & 34 \\
\hline $9 \mathrm{X}-4,115-150$ & 494.05 & IW & IId & & & & & & & & & & & & & 33,429 & 1,614 & 3,785 & 70 & 14 & 16 \\
\hline $9 X-5,75-79$ & 495.15 & FIS & IId & & & & & & & & & & & & & 23,618 & 3,189 & 9,741 & 42 & 23 & 35 \\
\hline Mean & & & & & & & 40 & 35 & 24 & & & & 39 & 32 & 29 & & & & 61 & 16 & 23 \\
\hline
\end{tabular}




\begin{tabular}{|c|c|c|c|c|c|c|c|c|c|c|c|c|c|c|c|c|c|c|c|c|c|}
\hline \multirow{3}{*}{$\begin{array}{l}\text { Core, section, } \\
\text { interval }(\mathrm{cm})\end{array}$} & \multirow{3}{*}{$\begin{array}{l}\text { Depth } \\
\text { (mbsf) }\end{array}$} & \multirow{3}{*}{$\begin{array}{l}\text { Sample } \\
\text { code }\end{array}$} & \multirow{3}{*}{$\begin{array}{r}\text { Strat. } \\
\text { unit }\end{array}$} & \multicolumn{6}{|c|}{ 4- to $8-\mu \mathrm{m}$ size fraction } & \multicolumn{6}{|c|}{$1-4-\mu \mathrm{m}$ size fraction } & \multicolumn{6}{|c|}{$<1-\mu \mathrm{m}$ size fraction } \\
\hline & & & & Integrated & peak ares & a (counts) & Estin & ed per & ntage & Integrated & peak are & a (counts) & Estin & ed perc & ntage & Integratec & peak area & $\mathrm{a}$ (counts) & Estim & ed perc & ntage \\
\hline & & & & Smectite & Illite & Kaolinite & $\% \mathrm{~S}$ & $\% \mathrm{I}$ & $\% \mathrm{~K}$ & Smectite & Illite & Kaolinite & $\% \mathrm{~S}$ & $\% \mathrm{I}$ & $\% \mathrm{~K}$ & Smectite & Illite & Kaolinite & $\% \mathrm{~S}$ & $\% \mathrm{I}$ & $\% \mathrm{~K}$ \\
\hline $10 \mathrm{X}-1,117-121$ & 499.27 & FIS & IIe & & & & & & & & & & & & & 24,191 & 2,584 & 4,914 & 55 & 23 & 22 \\
\hline $10 X-1,121-124$ & 499.31 & UND & IIe & 4,500 & 721 & 1,278 & 45 & 29 & 26 & 8,891 & 1,363 & 2,032 & 48 & 30 & 22 & 33,894 & 2,506 & 11,126 & 51 & 15 & 34 \\
\hline $10 \mathrm{X}-2,85-89$ & 500.45 & FIS & IIe & & & & & & & & & & & & & 32,422 & 5,734 & 16,440 & 37 & 26 & 37 \\
\hline $10 \mathrm{X}-2,94-98$ & 500.54 & UND & IIe & 11,646 & 2,192 & 4,262 & 40 & 30 & 29 & 10,644 & 2,034 & 3,548 & 41 & 31 & 27 & 25,019 & 2,354 & 6,814 & 52 & 20 & 28 \\
\hline $10 \mathrm{X}-3,59-62$ & 501.69 & UND & IIe & 6,669 & 1,670 & 3,403 & 33 & 33 & 34 & 10,025 & 2,694 & 5,904 & 31 & 33 & 36 & 24,979 & 3,166 & 11,919 & 41 & 21 & 39 \\
\hline $10 X-3,110-150$ & 502.20 & IW & IIe & & & & & & & & & & & & & 41,498 & 3,260 & 10,900 & 54 & 17 & 29 \\
\hline $10 \mathrm{X}-4,120-124$ & 503.80 & FIS & IIe & & & & & & & & & & & & & 29,418 & 5,418 & 20,352 & 32 & 24 & 44 \\
\hline $10 \mathrm{X}-4,126-130$ & 503.86 & UND & IIe & 3,095 & 922 & 1,090 & 35 & 41 & 24 & 11,219 & 1,697 & 3,632 & 44 & 27 & 29 & 36,436 & 1,898 & 4,274 & 69 & 14 & 16 \\
\hline $\begin{array}{l}\text { 10X-5, 53-56 } \\
\text { Mean }\end{array}$ & 504.63 & UND & IIe & 26,434 & 1,294 & 2,971 & $\begin{array}{l}70 \\
45\end{array}$ & $\begin{array}{l}14 \\
29\end{array}$ & $\begin{array}{l}16 \\
26\end{array}$ & 10,098 & 1,719 & 1,916 & $\begin{array}{l}49 \\
43\end{array}$ & $\begin{array}{l}33 \\
31\end{array}$ & $\begin{array}{l}18 \\
27\end{array}$ & 46,444 & 2,421 & 4,476 & $\begin{array}{l}71 \\
51\end{array}$ & $\begin{array}{l}15 \\
19\end{array}$ & $\begin{array}{l}14 \\
29\end{array}$ \\
\hline $11 \mathrm{X}-1,32-36$ & 508.02 & FIS & IIf & & & & & & & & & & & & & 23,787 & 4,156 & 8,215 & 42 & 29 & 29 \\
\hline $11 \mathrm{X}-1,66-70$ & 508.36 & UND & IIf & 24,558 & 1,703 & 3,958 & 63 & 17 & 20 & 16,467 & 1,117 & 3,054 & 61 & 17 & 23 & 40,794 & 1,127 & 4,924 & 74 & 8 & 18 \\
\hline 11X-1, 139-143 & 509.09 & FIS & IIf & & & & & & & & & & & & & 29,126 & 3,564 & 12,258 & 43 & 21 & 36 \\
\hline $11 \mathrm{X}-2,86-90$ & 510.06 & FIS & IIf & & & & & & & & & & & & & 28,573 & 5,826 & 21,898 & 30 & 24 & 46 \\
\hline $11 \mathrm{X}-3,51-55$ & 511.21 & FIS & IIf & & & & & & & & & & & & & 12,198 & 3,883 & 19,510 & 18 & 23 & 58 \\
\hline $11 \mathrm{X}-3,120-150$ & 511.90 & IW & IIf & & & & & & & & & & & & & 20,913 & 3,242 & 17,064 & 31 & 19 & 50 \\
\hline $11 X-4,43-47$ & 512.63 & UND & IIf & 10,327 & 2,855 & 6,064 & 30 & 34 & 36 & 13,999 & 3,397 & 10,593 & 29 & 28 & 43 & 25,637 & 3,994 & 15,663 & 35 & 22 & 43 \\
\hline $11 X-4,70-74$ & 512.90 & FIS & IIf & 10,021 & 2,035 & 0,004 & 30 & 34 & 30 & יצינ, 15ו & ה & צע, & 29 & 20 & 45 & 12,130 & 5,460 & 14,654 & 19 & 35 & 46 \\
\hline $11 \mathrm{X}-4,110-150$ & 513.30 & MK & IIf & & & & & & & & & & & & & 25,505 & 4,168 & 13,822 & 37 & 24 & 40 \\
\hline $11 X-5,13-17$ & 513.83 & UND & IIf & 5,718 & 2,889 & 3,907 & 23 & 46 & 31 & 7,393 & 3.634 & 5,645 & 22 & 44 & 34 & 26,600 & $\begin{array}{l}4,100 \\
4,307\end{array}$ & 12,579 & 39 & 25 & 36 \\
\hline $11 X-5,70-86$ & 514.40 & GZ & IIf & 4,611 & 1,428 & 2,439 & 30 & 38 & 32 & 2,256 & 1,321 & 2,254 & 19 & 44 & 37 & 14,272 & 3,975 & 9,322 & 29 & 33 & 38 \\
\hline Mean & & & & & & & 37 & 34 & 30 & & & & 33 & 33 & 34 & & & & 36 & 24 & 40 \\
\hline $12 \mathrm{X}-1,49-52$ & 517.89 & FIS & IIIa & & & & & & & & & & & & & 13,323 & 11,649 & 9,557 & 17 & 59 & 24 \\
\hline $12 \mathrm{X}-1,91-106$ & 518.31 & PB & IIIa & 2,794 & 1,420 & 3,152 & 19 & 38 & 43 & 4,764 & 1,877 & 4,602 & 22 & 35 & 43 & 18,010 & 5,770 & 16,201 & 25 & 31 & 44 \\
\hline $12 \mathrm{X}-2,32-33$ & 519.22 & UND & IIIa & 5,942 & 1,691 & 3,631 & 30 & 34 & 36 & 7,731 & 3,518 & 7,182 & 21 & 39 & 40 & 20,623 & 5,627 & 13,686 & 29 & 32 & 39 \\
\hline $12 \mathrm{X}-3,21-25$ & 520.61 & FIS & IIIa & & & & & & & & & & & & & 9,599 & 8,346 & 20,704 & 11 & 40 & 49 \\
\hline $12 X-3,40-57$ & 520.80 & HT & IIIa & 2,058 & 820 & 1,387 & 25 & 40 & 34 & 2,142 & 2,586 & 5,675 & 9 & 43 & 48 & 14,483 & 7,711 & 15,993 & 19 & 40 & 41 \\
\hline $12 X-4,4-7$ & 521.94 & FIS & IIIa & & & & & & & & & & & & & 16,850 & 11,473 & 26,713 & 15 & 40 & 46 \\
\hline $12 X-4,47-63$ & 522.37 & GZ & IIIa & 6,734 & 1,260 & 3,944 & 34 & 26 & 40 & 9,679 & 3,934 & 7,705 & 24 & 39 & 38 & 25,797 & 6,647 & 22,256 & 27 & 27 & 46 \\
\hline $12 \mathrm{X}-4,75-78$ & 522.65 & UND & IIIIa & $\begin{array}{l}0,144 \\
3,366\end{array}$ & $\begin{array}{l}1,200 \\
1,202\end{array}$ & 2,208 & $\begin{array}{l}34 \\
27\end{array}$ & 38 & 35 & $\begin{array}{l}4,045 \\
4,0 / 9\end{array}$ & $\begin{array}{l}3,634 \\
1,610\end{array}$ & 2,825 & 25 & 40 & 35 & 25,221 & $\begin{array}{l}\quad, 047 \\
5,529\end{array}$ & $\begin{array}{l}22,750 \\
19,700\end{array}$ & 29 & 25 & $\begin{array}{l}40 \\
45\end{array}$ \\
\hline $12 \mathrm{X}-4,110-150$ & 523.00 & IW & IIIa & & & & & & & & & & & & & 15,390 & 4,546 & 11,320 & 27 & 32 & 40 \\
\hline Mean & & & & & & & 27 & 35 & 38 & & & & 20 & 39 & 41 & & & & 22 & 36 & 42 \\
\hline $12 \mathrm{X}-5,49-52$ & 523.89 & FIS & IIIb & & & & & & & & & & & & & 20,836 & 12,181 & 32,326 & 16 & 36 & 48 \\
\hline 12X-CC, $4-15$ & 525.77 & $\mathrm{AM}$ & IIIb & 2,116 & 1,490 & 2,233 & 17 & 48 & 36 & 2,858 & 3,607 & 6,581 & 9 & 47 & 43 & 21,981 & 9,327 & 20,965 & 22 & 37 & 41 \\
\hline $13 \mathrm{X}-2,16-19$ & 528.46 & FIS & IIIb & & & & & & & & & & & & & 8,360 & 10,971 & 27,139 & 8 & 41 & 51 \\
\hline $13 \mathrm{X}-3,12-15$ & 529.92 & FIS & IIIlb & & & & & & & & & & & & & 11,367 & 12,236 & 30,801 & 9 & 40 & 51 \\
\hline $13 \mathrm{X}-3,60-70$ & 530.40 & FIS & IIIb & & & & & & & & & & & & & 3,655 & 14,528 & 27,114 & 3 & 50 & 47 \\
\hline $13 \mathrm{X}-3,60-70$ & 530.40 & $\mathrm{AF}$ & IIIb & 2,951 & 1,017 & 2,286 & 25 & 35 & 39 & 4,363 & 2,241 & 5,680 & 18 & 36 & 46 & 16,338 & 8,366 & 17,325 & 19 & 40 & 41 \\
\hline $13 \mathrm{X}-3,123-126$ & 531.03 & UND & IIIb & 3,393 & 1,656 & 4,384 & 18 & 35 & 47 & 4,679 & 2,550 & 6,430 & 17 & 37 & 46 & 19,007 & 6,892 & 30,069 & 18 & 26 & 56 \\
\hline $13 \mathrm{X}-4,16-19$ & 531.46 & UND & IIIb & 3,642 & 1,008 & 2,263 & 30 & 33 & 37 & 3,884 & 1.921 & 4,806 & 18 & 36 & 45 & 11,167 & 5,070 & 24,233 & 14 & 25 & 61 \\
\hline 13X-4, 115-15-150 & $\begin{array}{l}531.40 \\
532.45\end{array}$ & IW & IIIIb & 3,642 & 1,008 & 2,203 & 30 & 33 & 31 & 3,884 & 1,921 & 4,800 & 18 & 30 & 45 & $\begin{array}{r}11,10 / \\
6,923\end{array}$ & 8,348 & $\begin{array}{l}24,233 \\
13,289\end{array}$ & $\begin{array}{l}14 \\
10\end{array}$ & 50 & $\begin{array}{l}01 \\
40\end{array}$ \\
\hline $13 \mathrm{X}-5,26-42$ & 533.06 & HT & IIIb & 1,806 & 3,600 & 7,129 & 6 & 47 & 47 & 2,418 & 3,407 & 1,046 & 67 & 37 & 57 & 7,969 & 7,637 & 28,275 & 8 & 32 & 59 \\
\hline $13 \mathrm{X}-6,21-25$ & 534.51 & UND & IIIb & $\begin{array}{l}3,041 \\
3,041\end{array}$ & 610 & , & 41 & 33 & 25 & 5,47 & 2219 & $5,26-10$ & 22 & 36 & 42 & 1803 & 6238 & 434667 & 19 & 10 & 72 \\
\hline $13 \mathrm{X}-7,36-51$ & 536.16 & GZ & $\mathrm{IIIb}$ & 1,847 & $\begin{array}{r}1,082 \\
10\end{array}$ & 1,199 & $\begin{array}{l}11 \\
22\end{array}$ & 50 & 28 & 5,3 & 1,704 & 6,4 & 21 & 27 & 51 & $\begin{array}{l}1,279 \\
1,279\end{array}$ & 3,241 & 431, & 15 & 11 & 73 \\
\hline 13X-CC, $15-25$ & 536.46 & JA & $\mathrm{IIIb}$ & $\begin{array}{l}1,0+1 \\
1,364\end{array}$ & $\begin{array}{l}1,002 \\
926\end{array}$ & 2,008 & 15 & 41 & 44 & 2,893 & 3,939 & 7,4 & $\begin{array}{r}1 \\
9\end{array}$ & 47 & 44 & 8,033 & 6,893 & 12,132 & 13 & 46 & 41 \\
\hline $14 \mathrm{X}-1,45-4$ & 536.55 & UND & IIIb & 3,968 & 1,238 & 2,512 & 28 & 36 & 36 & 4,764 & 2,023 & 2,665 & 26 & 44 & 29 & 12,277 & 17,719 & 35,737 & 8 & 46 & 46 \\
\hline $14 \mathrm{X}-3,110-150$ & 540.20 & MK & IIIb & & & & & & & & & & & & & 7,496 & 9,471 & 20,214 & 9 & 44 & 47 \\
\hline $14 \mathrm{X}-4,26-30$ & 540.86 & UND & IIIb & 2,748 & 1,059 & 891 & 31 & 48 & 20 & 2,583 & 1,170 & 1,672 & 24 & 44 & 32 & 20,674 & 5,490 & 21,618 & 24 & 26 & 50 \\
\hline $14 \mathrm{X}-4,122-126$ & 541.82 & UND & IIIb & 3,171 & 774 & 856 & 40 & 39 & 21 & 11,026 & 1,630 & 8,783 & 31 & 19 & 50 & 9,106 & 1,604 & 27,401 & 13 & 9 & 78 \\
\hline $14 \mathrm{X}-5,120-150$ & 543.30 & IW & IIIb & & & & & & & & & & & & & 8,680 & 7,828 & 21,064 & 11 & 38 & 51 \\
\hline $14 \mathrm{X}-6,36-37$ & 543.96 & GZ & IIIb & 1,700 & 688 & 2,335 & 19 & 30 & 51 & 3,757 & 1,158 & 5,495 & 19 & 24 & 57 & 24,740 & 3,398 & 36,062 & 22 & 12 & 65 \\
\hline $14 \mathrm{X}-6,52-70$ & 544.12 & $\mathrm{AM}$ & IIIb & 5,019 & 910 & 3,274 & 33 & 24 & 43 & 6,754 & 1.477 & 3,983 & 33 & 29 & 39 & 23,522 & 3,098 & 15,214 & 35 & 19 & 46 \\
\hline $14 \mathrm{X}-6,79-92$ & 544.39 & SP & IIIJ & 5,781 & 1.742 & 4,525 & 27 & 32 & 42 & $\begin{array}{r}12,754 \\
12952\end{array}$ & 1.488 & $\begin{array}{r}11,503 \\
11.525\end{array}$ & 31 & 14 & 55 & 20,659 & 2,756 & 21,846 & 27 & 15 & 58 \\
\hline $15 \mathrm{X}-1,61-65$ & 546.01 & UND & IIIb & 1,557 & 1,969 & 6,076 & 7 & 36 & 56 & 18,773 & $\begin{array}{l}1,400 \\
2,758\end{array}$ & 20,142 & 27 & 16 & 57 & 24,012 & 5,919 & $\begin{array}{l}21,040 \\
42,168\end{array}$ & 18 & 18 & 64 \\
\hline $15 \mathrm{X}-4,61-67$ & 550.51 & GZ & $\mathrm{IIIb}$ & 3,459 & 2,779 & 4,827 & 14 & 46 & 40 & 7,765 & 2,787 & 4,982 & 27 & 39 & 35 & 15,131 & 5,542 & 9,843 & 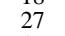 & 39 & 35 \\
\hline $15 X-5,75$ & 552.15 & UND & IIIb & 4,462 & 1.842 & 3,428 & 24 & 39 & 37 & 6,2 & 3,67 & 9,39 & 16 & 37 & 47 & 14,490 & 7,523 & 21.500 & 17 & 34 & 49 \\
\hline 15$\rangle$ & 553.35 & UND & IIIb & 3863 & $\begin{array}{l}1,0+2 \\
904\end{array}$ & 1,210 & 39 & 37 & 24 & 7,877 & 1,983 & 5,697 & 29 & 29 & 42 & 19, & & & 27 & 18 & 55 \\
\hline $\begin{array}{l}15 \mathrm{X}-0, \\
15,\end{array}$ & 554.00 & IW & IIIb & & & & & & & & & & & & & & 2, & & 17 & 33 & 50 \\
\hline $16 \mathrm{X}-2,110-150$ & 557.40 & IW & IIIb & & & & & & & & & & & & & 9,428 & 1,876 & 8,621 & 28 & 22 & 50 \\
\hline $16 \mathrm{X}-5,119-135$ & 561.99 & PB & IIIIb & 4,196 & 713 & 1,506 & 42 & 28 & 30 & 4,608 & 1,166 & 3,794 & 27 & 28 & 45 & 8,942 & 1,957 & 13,495 & 20 & 18 & 62 \\
\hline $16 \mathrm{X}-6,32-36$ & & UND & & 4,896 & 1,753 & 3,071 & & 39 & & 4,930 & 5,331 & 12,534 & 10 & 42 & & & & 29,047 & 24 & & 42 \\
\hline
\end{tabular}


Table 1 (continued).

\begin{tabular}{|c|c|c|c|c|c|c|c|c|c|c|c|c|c|c|c|c|c|c|c|c|c|}
\hline \multirow{3}{*}{$\begin{array}{l}\text { Core, section, } \\
\text { interval }(\mathrm{cm})\end{array}$} & \multirow{3}{*}{$\begin{array}{l}\text { Depth } \\
\text { (mbsf) }\end{array}$} & \multirow{3}{*}{$\begin{array}{l}\text { Sample } \\
\text { code }\end{array}$} & \multirow{3}{*}{$\begin{array}{r}\text { Strat. } \\
\text { unit }\end{array}$} & \multicolumn{6}{|c|}{ 4- to 8 - $\mu \mathrm{m}$ size fraction } & \multicolumn{6}{|c|}{ 1-4- $\mu \mathrm{m}$ size fraction } & \multicolumn{6}{|c|}{$<1-\mu \mathrm{m}$ size fraction } \\
\hline & & & & \multicolumn{3}{|c|}{ Integrated peak area (counts) } & \multicolumn{3}{|c|}{ Estimated percentage } & \multicolumn{3}{|c|}{ Integrated peak area (counts) } & \multicolumn{3}{|c|}{ Estimated percentage } & \multicolumn{3}{|c|}{ Integrated peak area (counts) } & \multicolumn{3}{|c|}{ Estimated percentage } \\
\hline & & & & Smectite & Illite & Kaolinite & $\% \mathrm{~S}$ & $\% \mathrm{I}$ & $\% \mathrm{~K}$ & Smectite & Illite & Kaolinite & $\% \mathrm{~S}$ & $\% \mathrm{I}$ & $\% \mathrm{~K}$ & Smectite & Illite & Kaolinite & $\% \mathrm{~S}$ & $\% \mathrm{I}$ & $\% \mathrm{~K}$ \\
\hline $16 \mathrm{X}-6,50-61$ & 562.80 & JA & $\mathrm{IIIb}$ & 1,738 & 1,924 & 3,926 & 10 & 45 & 45 & 6,416 & 4,042 & 10,1 & 15 & 38 & 47 & 31,396 & 7,376 & 18, & 32 & 30 & 37 \\
\hline $17 \mathrm{X}-2,97-99$ & 566.57 & UND & IIIIb & 4,701 & 1,283 & 2,238 & 33 & 36 & 31 & 8,057 & 2,063 & 4,874 & 31 & 32 & 37 & 27, & 9,053 & 29,3 & 22 & 30 & 48 \\
\hline $17 \mathrm{X}-2,120-150$ & 566.80 & IW & IIIb & & & & & & & & & & & Jz & & 14,213 & 2,636 & 11,583 & 30 & 22 & 48 \\
\hline $17 \mathrm{X}-6,48-53$ & 572.08 & UND & IIIb & 4,025 & 894 & 1,451 & 38 & 34 & 28 & 4,047 & 1,496 & 3,103 & 25 & 37 & 38 & 9,210 & 1,492 & 12,319 & 23 & 15 & 62 \\
\hline $17 \mathrm{X}-6,67-78$ & 572.27 & $\mathrm{AM}$ & IIIlb & 7,618 & 3,552 & 8,841 & 19 & 36 & 45 & 4,310 & 2,920 & 8,090 & 13 & 36 & 50 & 11,106 & 7,842 & 13,046 & 16 & 46 & 38 \\
\hline 17X-6, $133-135$ & 572.93 & UND & IIIIb & 4,599 & 1,522 & 2,791 & 28 & 37 & 34 & 2,183 & 5,475 & 10,500 & 5 & 49 & 47 & 21,069 & 10,840 & 23,894 & 19 & 39 & 43 \\
\hline $18 \mathrm{X}-2,23-25$ & 575.03 & UND & IIIb & 4,687 & 1,117 & 1,285 & 40 & 38 & 22 & 6,006 & 2,198 & 4,5 & 25 & 37 & 38 & 17,633 & 4,623 & 20,809 & 23 & 24 & 54 \\
\hline $18 \mathrm{X}-4,33-38$ & 577.90 & UND & IIIb & 3,351 & 1,081 & 2,395 & 27 & 35 & 38 & 12,301 & 2,855 & 8,439 & 30 & 28 & 42 & 14,262 & 3,376 & 11,249 & 28 & 27 & 45 \\
\hline $18 \mathrm{X}-4,110-150$ & 578.90 & IW & IIIb & & & & & & & & & & & & & 10,839 & 5,581 & 13,183 & 18 & 38 & 44 \\
\hline $18 \mathrm{X}-5,139-150$ & 580.69 & PH & IIIb & 1,404 & 418 & 1,710 & 22 & 26 & 53 & 4,029 & 1,733 & 5,634 & 18 & 31 & 51 & 16,499 & 1,984 & 40,638 & 16 & 8 & 77 \\
\hline $19 \mathrm{X}-2,26-30$ & 584.56 & UND & IIIb & 3,936 & 727 & 929 & 45 & 33 & 21 & 8,298 & 1,698 & 3,133 & 39 & 32 & 29 & 11,904 & 2,406 & 10,564 & 28 & 23 & 50 \\
\hline $19 \mathrm{X}-4,56-58$ & 587.86 & UND & IIIIb & 6,588 & 1,566 & 3,696 & 33 & 31 & 37 & 5,269 & 1,751 & 5,420 & 23 & 30 & 47 & 34,920 & 5,399 & 30,895 & 30 & 18 & 52 \\
\hline $19 \mathrm{X}-4,110-150$ & 588.40 & IW & IIIIb & & 1,000 & , & 53 & נI & & 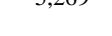 & & & & so & 41 & 17,791 & 7,918 & 13,694 & 23 & 41 & 36 \\
\hline $19 \times-6,49-50$ & 590.78 & HT & IIIlb & 3,244 & 1,590 & 1,484 & 26 & 51 & 24 & 2,564 & 1,470 & 4,605 & 15 & 33 & 52 & 20,761 & 6,269 & 17,060 & 26 & 31 & 43 \\
\hline $19 \mathrm{X}-7,40-43$ & 592.20 & UND & IIIb & 2,678 & 3,300 & 3,736 & 11 & 57 & 32 & 4,322 & 5,454 & 8,817 & 10 & 50 & 40 & 34,623 & 11,134 & 23,116 & 28 & 36 & 37 \\
\hline Mean & & & & & & & 26 & 38 & 36 & & & & 21 & 34 & 45 & & & & 20 & 29 & 51 \\
\hline
\end{tabular}

Notes: Sample codes UND and FIS correspond to discrete intervals. IW indicates trimming from interstitial water whole-rounds. All other codes correspond to miscellaneous whole-round trimmings. 


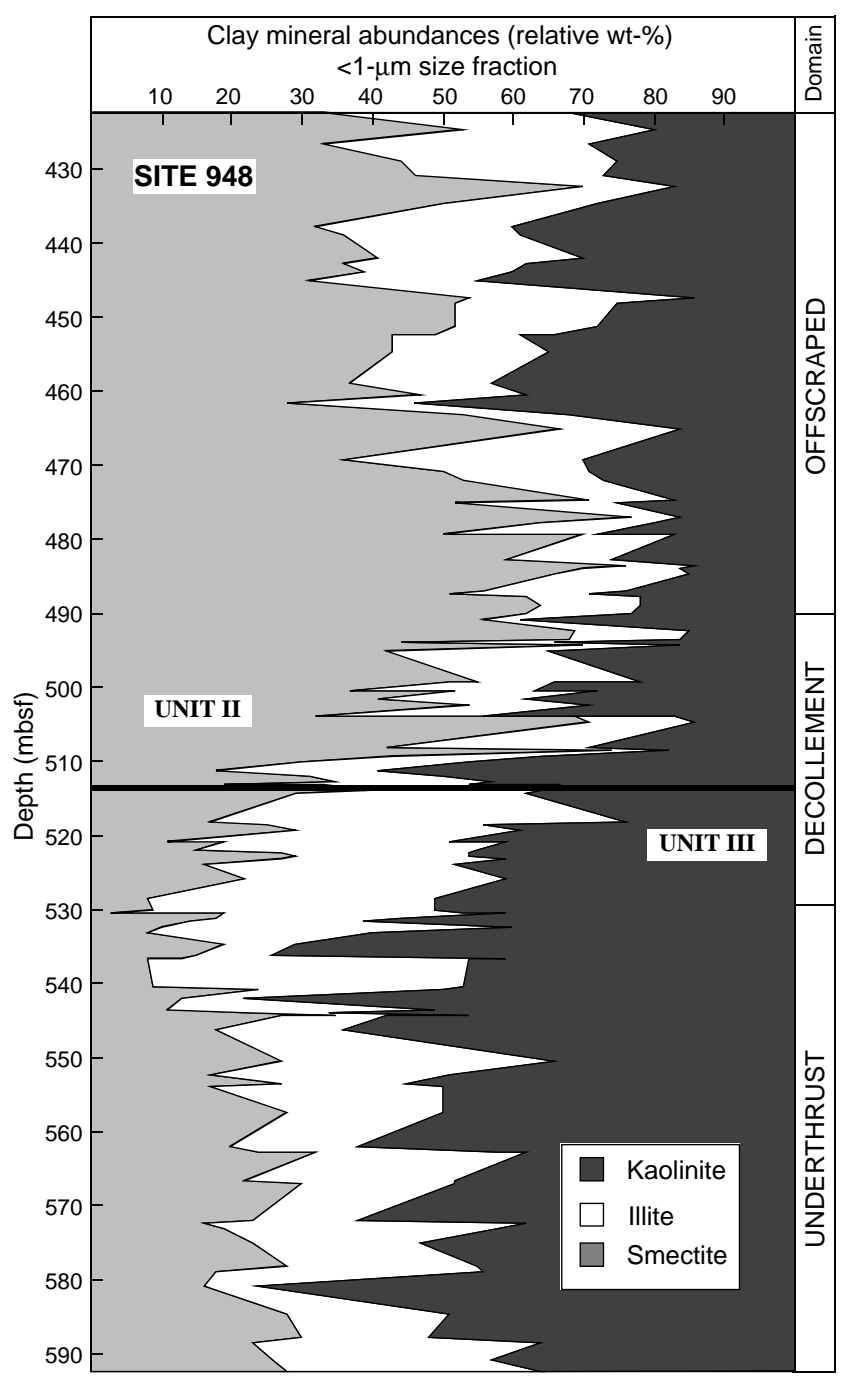

Figure 6. Relative abundances of smectite, illite, and kaolinite in clay-sized fraction $(<1 \mu \mathrm{m})$ of samples from Site 948 . See Table 1 for specific sample numbers, depths, integrated peak areas, and clay-mineral percentages based

cal electron microscopy (AEM). With these caveats in mind, we analyzed thirty-eight samples from Hole $948 \mathrm{~B}$ by SEM/EDS to determine whether or not chemical variations are significant in the vicinity of the décollement zone. Table 4 displays all of the results.

Several obvious changes in clay composition occur at the lithologic boundary between Units II and III and down the décollement zone (Fig. 13). In general, clay-sized assemblages from Unit II have lower weight percentages of $\mathrm{Al}_{2} \mathrm{O}_{3}$ and higher weight percentages of $\mathrm{SiO}_{2}, \mathrm{Fe}_{\text {total }}, \mathrm{K}_{2} \mathrm{O}, \mathrm{MgO}$, and $\mathrm{TiO}_{2}$ as compared to samples from Unit III. Contents of alumina increase across the lithologic boundary, which is to be expected given the well-documented increase in the relative abundance of kaolinite $\left[\mathrm{Al}_{2} \mathrm{SiO}_{2} \mathrm{O}_{5}(\mathrm{OH})_{4}\right]$ over the same depth interval. Silica content increases within the smectite-rich interval at the top of the décollement and fluctuates irregularly within the interbeds of turbidites and pelagic claystones of Unit III. The amount of $\mathrm{K}_{2} \mathrm{O}$ decreases gradually downsection within Unit II and fluctuates irregularly within Unit III. Values of $\mathrm{MgO}$ display considerable scatter throughout Unit II but maintain a fairly narrow range of 1.2-2.4 wt\% within Unit III (Fig. 13).

Chemical changes in sediment composition are often described in terms of elemental atomic ratios or oxide ratios of the major-element constituents relative to that of an element of low mobility, such as aluminum, particularly when assessing the effects of diagenesis. Figure 14 shows the relevant ratios of oxide weight percentage for Site 948. These data display a clear decrease in $\mathrm{SiO}_{2} / \mathrm{Al}_{2} \mathrm{O}_{3}$ from Unit II to Unit III, a negative gradient in $\mathrm{K}_{2} \mathrm{O} / \mathrm{Al}_{2} \mathrm{O}_{3}$ within Unit II, and a subtle but steady decrease in $\mathrm{TiO}_{2} / \mathrm{Al}_{2} \mathrm{O}_{3}$ throughout the cored section. Values of $\mathrm{CaO} / \mathrm{Al}_{2} \mathrm{O}_{3}$ are generally below 0.1 , but increase locally in response to the content of calcareous nannofossils; large spikes in calcium content are obvious, for example, within the chalky turbidites of Unit III. Values of $\mathrm{Fe}_{\text {total }} / \mathrm{Al}_{2} \mathrm{O}_{3}$ and $\mathrm{MgO} / \mathrm{Al}_{2} \mathrm{O}_{3}$ are slightly higher in Unit II than in Unit III (Fig. 14).

Relative proportions of smectite, illite, and kaolinite should be the most straightforward variables to influence the geochemistry of claysized constituents, and Hathon and Underwood (1991) showed that the best chemical indicators of clay mineralogy are $\mathrm{Al}_{2} \mathrm{O}_{3}, \mathrm{SiO}_{2}$, and $\mathrm{K}_{2} \mathrm{O}$. To test these contentions, we constructed regression plots of major-oxide percentages and major-oxide ratios (relative to alumina) vs. the estimated abundance of each clay mineral within the $<1-\mu \mathrm{m}$ size fraction. For a population size of 43 , correlation coefficients greater than 0.40 are statistically significant at the $99.5 \%$ confidence level.

Minerals within the dioctahedral smectite group can vary considerably in their contents of $\mathrm{Mg}^{2+}, \mathrm{Na}^{+}, \mathrm{K}^{+}$, and $\mathrm{Fe}^{3+}$. End-member minerals include montmorillonite ( $\mathrm{Na}-\mathrm{Mg}$-rich), beidellite ( $\mathrm{Na}-\mathrm{Fe}-\mathrm{rich})$, and nontronite (K-Fe-rich). Despite the presence of non-clay minerals, Figure 15 shows that statistically significant correlations exist between values of \% smectite and weight percentage values of all oxides except $\mathrm{CaO} . \mathrm{SiO}_{2}, \mathrm{Fe}_{\text {total }}, \mathrm{MgO}$, and $\mathrm{TiO}_{2}$ contents all display positive correlations, whereas $\mathrm{Al}_{2} \mathrm{O}_{3}$ and $\mathrm{K}_{2} \mathrm{O}$ contents decrease with increasing smectite content. The best statistical match is for alumina vs. $\%$ smectite $(\mathrm{r}=0.79)$. When expressed in terms of oxide ratios, only $\mathrm{SiO}_{2} / \mathrm{Al}_{2} \mathrm{O}_{3}, \mathrm{Fe}_{\text {total }} / \mathrm{Al}_{2} \mathrm{O}_{3}$, and $\mathrm{TiO}_{2} / \mathrm{Al}_{2} \mathrm{O}_{3}$ yield correlations with $\%$ smectite that are meaningful (Fig. 15). The correlation for silica/ alumina vs. \% smectite is particularly strong, with a correlation coefficient of 0.86 . Conversely, the r-value for the regression between $\mathrm{K}_{2} \mathrm{O} / \mathrm{Al}_{2} \mathrm{O}_{3}$ and $\%$ smectite is only 0.23 .

The clay mineral illite generally contains more $\mathrm{Si}$ and $\mathrm{Mg}$ and less $\mathrm{K}$ than ideal muscovite, which has the chemical formula $\mathrm{KAl}_{2}\left(\mathrm{Si}_{3} \mathrm{Al}\right) \mathrm{O}_{10}(\mathrm{OH})_{2}$. Given the previous findings of Hathon and Underwood (1991), the best chemical indicator of illite and/or detrital muscovite content in a mixture of clay-sized minerals should be $\mathrm{K}_{2} \mathrm{O}$. Nevertheless, our regression analyses failed to produce statistically significant correlation coefficients (Fig. 16). $\mathrm{TiO}_{2} / \mathrm{Al}_{2} \mathrm{O}_{3}$ produced the best correlation, despite the likely contamination by titanomagnetite and/or titanium oxide, but the r-value for that regression is only 0.43 .

The SEM/EDS study of Hathon and Underwood (1991) did not include analyses of kaolinite, but its chemical signature should be easy to recognize. Relative percentages of kaolinite from Site 948 correlate effectively with weight percentages of all major oxides except $\mathrm{TiO}_{2}$ and $\mathrm{CaO}$ (Fig. 17). As expected, there is a robust positive correlation with $\mathrm{Al}_{2} \mathrm{O}_{3}(\mathrm{r}=0.82)$. There is a weak positive correlation with $\mathrm{K}_{2} \mathrm{O}$, and regression lines for $\mathrm{SiO}_{2}, \mathrm{Fe}_{\text {total }}$, and $\mathrm{MgO}$ have negative slopes. Oxide ratios display excellent negative correlations between $\%$ kaolinite and $\mathrm{SiO}_{2} / \mathrm{Al}_{2} \mathrm{O}_{3}(\mathrm{r}=0.85)$ and $\mathrm{Fe}_{\text {total }} / \mathrm{Al}_{2} \mathrm{O}_{3}(\mathrm{r}=$ 0.70), plus a weak correlation with $\mathrm{TiO}_{2} / \mathrm{Al}_{2} \mathrm{O}_{3}$ (Fig. 17).

\section{DISCUSSION}

Three fundamental questions regarding clay minerals in the Barbados Ridge subduction zone require additional scrutiny.

1. What effect does clay mineralogy have on the location and behavior of major structural features, especially the basal décollement of the accretionary prism? 


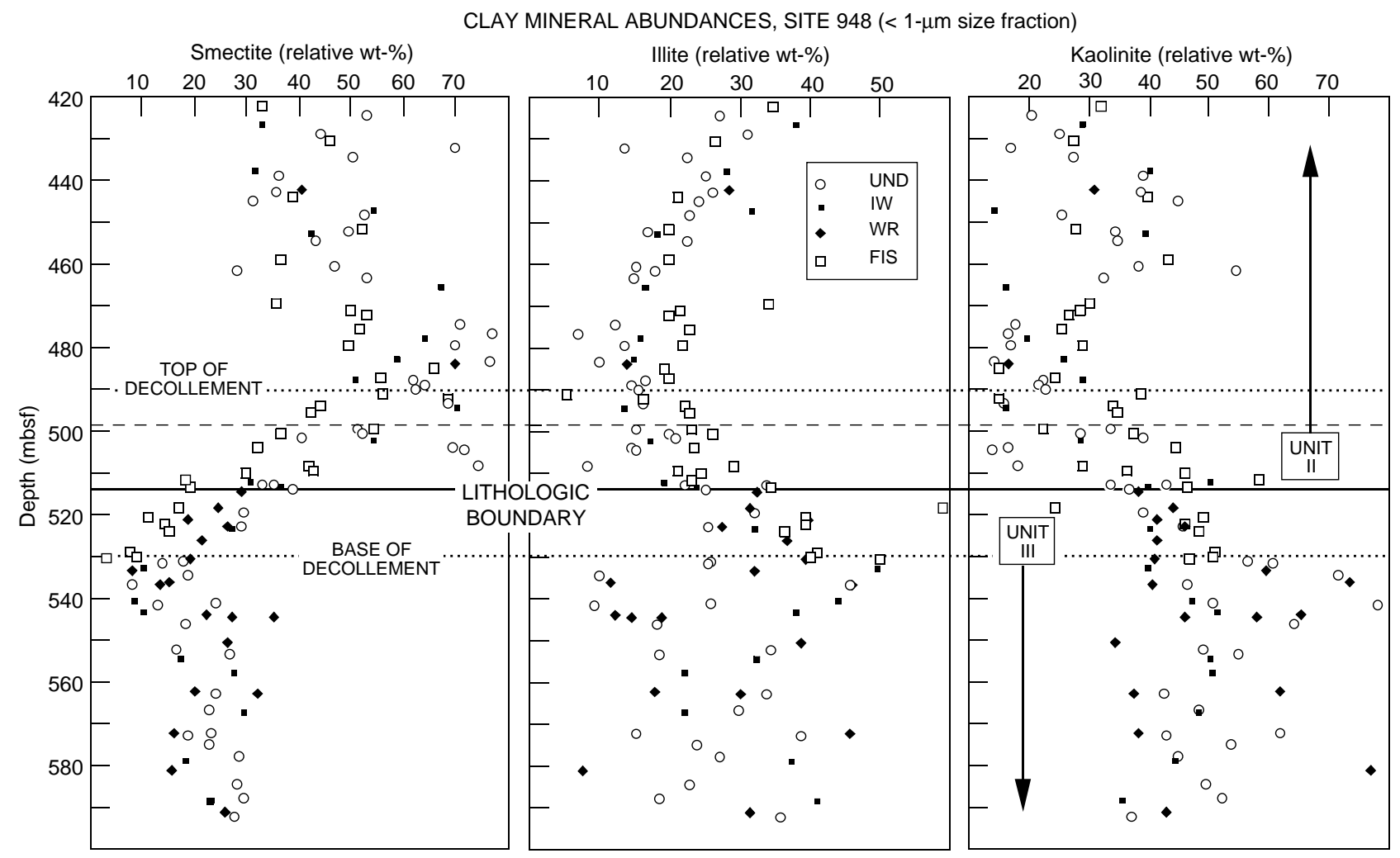

Figure 7. Individual depth profiles of relative abundance for smectite, illite, and kaolinite in clay-sized fraction $(<1-\mu \mathrm{m})$ of samples from Site 948 . Data for different types of specimens are plotted separately; UND = discrete interval; IW = trimmings from interstitial water whole-rounds; WR $=$ trimmings from miscellaneous whole-rounds; FIS = residue from discrete interval used in grain-size analysis.

2. What is the origin of the smectite-rich stratigraphic interval near the top of the décollement zone (detrital, authigenic, or both)?

3. Has the diagenetic transformation of smectite to illite occurred in situ, and if so, which of the structural domains and stratigraphic units have been affected?

The results of TEM and oxygen isotope analyses of bulk clays (Buatier et al., 1992; Tribble and Yeh, 1994) are consistent with four sources or types of smectite-rich clay: (1) subaerial weathering of volcanic protoliths and glass shards; (2) alteration of ash layers and dispersed ash on or near the seafloor; (3) erosion from continental rocks exposed to intermediate and/or advanced levels of diagenesis; and (4) in situ illitization. The challenge now is to determine which of these mechanisms might be most influential within specific lithostratigraphic intervals or structural domains. In all cases, mixtures of sources and combinations of formational mechanisms need to be considered.

\section{Influence of Clay Mineralogy on Position of Décollement}

One result common to all of the prior studies of clay minerals within the Barbados subduction zone is the occurrence of smectiterich claystones within and/or immediately above the top of the décollement zone (Latouche and Maillet, 1984; Pudsey, 1984; Schoonmaker, 1986; Tribble, 1990; Capet et al., 1990). Percentages of smectite also are unusually high within correlative stratigraphic intervals at the two oceanic reference sites (i.e., lower to middle Miocene radiolarian claystones at Sites 543 and 672), where deformation fabrics are indicative of an incipient décollement. It is clear that smectite-rich strata form intervals of preferential weakness (Vrolijk, 1990), but not all décollements and thrust faults propagate through smectite-rich sediments. Well-documented examples that violate this paradigm in- clude the Vanuatu collision zone, where imbricate thrust faults have developed along kaolinite-rich horizons (Reid et al., 1994), and the Nankai Trough, where the décollement is located in the middle of a homogeneous succession of smectite-poor, hemipelagic mudstone (Underwood et al., 1993b).

Data from our study show very clearly that the position of the Barbados décollement zone is affected by an interval of smectite-rich sediment (Figs. 7, 11). This interval, however, is fairly thick (approximately $35 \mathrm{~m}$ at Site 948), and the smectite content is erratic throughout $(48 \%-78 \%)$. We are unable, therefore, to select one smectite-rich marker-bed within this interval as the preferred target for seaward propagation of the décollement. Instead, the upper boundary of the décollement probably shifts its position both vertically and along strike. On the other hand, we can locate the base of the décollement zone precisely using clay-mineralogy data from Site 948 . Unlike previous studies, the close spacing of our samples defines consistent mineral profiles down the décollement zone. Smectite content decreases gradually and reaches minimum values of less than $10 \%$ at the base of the décollement, whereas illite content increases gradually to values of $50 \%$ over the same $40 \mathrm{~m}$ (Fig. 7). We suggest that the position of the décollement is controlled as much by the smectite minimum at its base as it is by the high content of expandable clays at its top. Claystones richest in illite (and kaolinite, to a lesser degree) should possess the highest values of shear strength, so downward propagation of the shear zone should terminate consistently where the claystones attain their greatest amounts of shear strength. Given the irregular fluctuations in clay mineralogy near the top of the décollement zone; however, its upper boundary is more diffuse.

\section{Clay Mineral Provenance}

The origin and detailed compositional character of smectite-rich clays in the Barbados subduction zone remain controversial. Tribble 
CLAY MINERAL DISTRIBUTION BY GRAIN SIZE
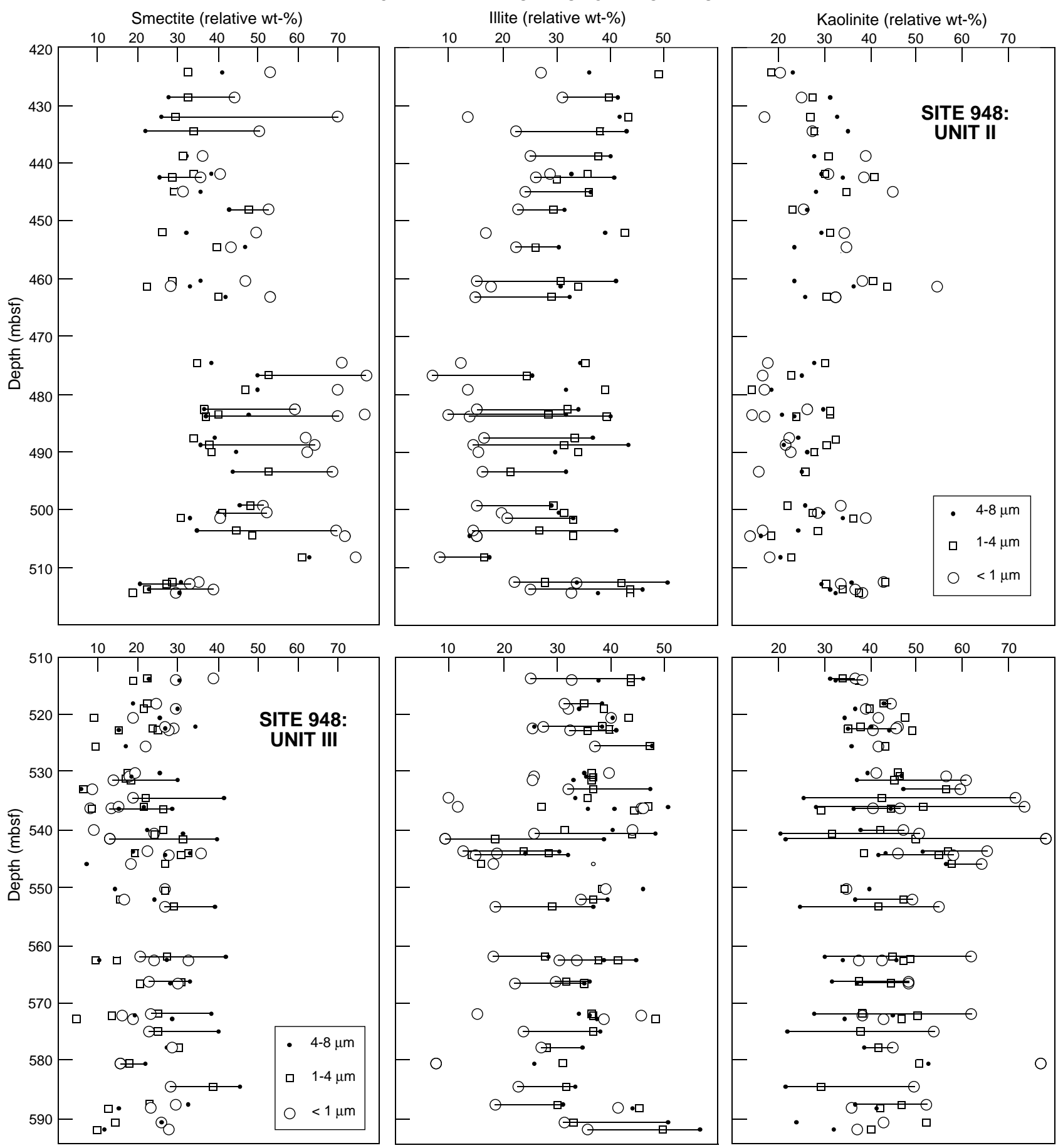

Figure 8. Partitioning of clay minerals as a function of grain size, lithofacies Units II and III, Site 948. Tie lines indicate that the specimen displays systematic changes in relative abundance across three size fractions. See Table 1 for pertinent data.

(1990) argued in favor of a mechanism in which smectite forms through alteration of volcanic ash at or near the seafloor. Capet et al. (1990), in contrast, argued that all of the clay minerals throughout the study area were derived from weathering and erosion of subaerial exposures in South America, as well as volcanic islands of the Lesser Antilles. As summarized by Chamley (1989), smectite-rich marine sediments can form in a variety of ways, and in all likelihood, several sources and processes contributed to the smectite and I/S budgets of Barbados sediments. One way to assess the respective contributions of different source areas and mechanisms of formation is to examine the compositions of late Quaternary near-surface sediments with known provenance and known dispersal pathways. The physical mechanisms responsible for sedimentation in deep-marine environments during the Oligocene and Miocene probably were the same as today: suspension fall-out from surface waters, sediment gravity flow, and remobilization within the bottom nepheloid layer by thermo-haline currents. We know that fresh tuffaceous and pumaceous debris is mixed thoroughly into near-surface sediments of the present-day forearc, and homogenization of detrital constituents is enhanced by bottom currents and bioturbation (Cleary et al., 1984). 


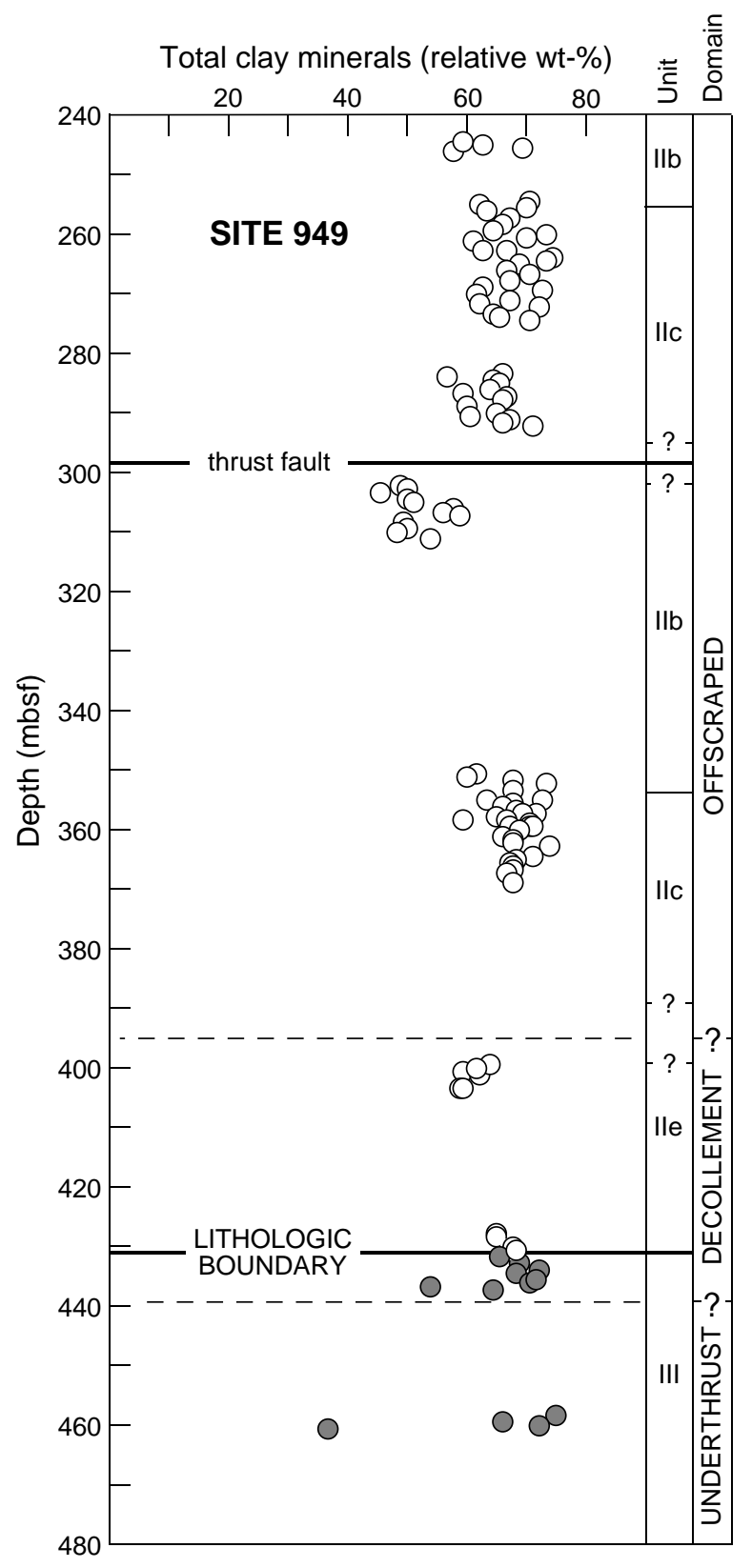

Figure 9. Relative abundance of total clay minerals in bulk samples from Site 949. See Shipboard Scientific Party (1995b) for additional data.

One circumstantial indicator of changing clay-mineral provenance comes from trends in grain-size partitioning. In general, detrital constituents should increase in relative abundance within coarser size fractions, whereas authigenic clays should be finer in grain size. This criterion, for example, was used by Buatier et al. (1992) to help discriminate between larger detrital illite grains and "neoformed" illite crystallites. Additional subdivisions of the clay-sized fractions might provide us with more definitive results, but existing data show that the partitioning behavior of clay minerals changes from Unit II to Unit III (Fig. 8). This is not an artifact of sample preparation because samples from both units were treated the same way. Illite displays consistent increases in relative abundance in coarser size fractions. Bentonite-like samples within Unit II show consistent increases of $\%$ smectite in the finest size fraction, which tends to support the contention of sporadic increases in the fine-grained authigenic contribution superimposed on a detrital background. Samples from Unit III, conversely, show the opposite trend with relative percentages of smectite increasing in the coarser size fraction. We believe that this shift in partitioning behavior is one of several manifestations of changing detrital provenance across the lithologic boundary.

Perhaps the most logical candidates for an authigenic origin of expandable clay are local layers of nearly pure smectite (bentonites). Marine bentonite beds can form either through direct subaqueous alteration of ash-fall deposits or by alteration of ash-rich sediments that were first eroded and transported from subaerial exposures (Hodder et al., 1993; Naish et al., 1993). Discrete layers of air-fall ash and pyroclastic turbidites are easily recognized in both near-surface sediments (Sigurdsson et al., 1980; Cleary et al., 1984; Wright, 1984; Reid et al., 1996) and the DSDP/ODP cores (Biju-Duval, Moore, et al., 1984; Mascle, Moore, et al., 1988; Shipley, Ogawa, Blum, et al., 1995). Most of the Barbados Ridge sediment, by volume, however, consists of heterogeneous mixtures of clay minerals, biogenic constituents, and detrital quartz and feldspar. Relative proportions of these constituents vary considerably in the Oligocene-Miocene sediments. Some of the disseminated smectite certainly could have formed by complete replacement of disseminated ash. It is important to note that Buatier et al. (1992) were unable to detect any disseminated volcanic glass in their TEM images, but they left open the possibility of complete alteration, even in the shallowest samples. Perhaps such contributions of ash were important locally, but Quaternary analogues lead us to believe that the disseminated ash was mixed with larger amounts of detrital clay minerals, including detrital smectite. Did the detrital smectite come from the Lesser Antilles arc or the continent of South America? Several indirect lines of evidence can be used to argue for multiple sources.

Holocene soils on the volcanic island of Martinique are known to contain only smectite and kaolinite in their clay-mineral assemblages (Latouche and Maillet, 1984; Gandais, 1987; Capet et al., 1990). Near-surface shallow-water sediments from the back-arc side of Martinique contain an average of $72 \%$ smectite and $28 \%$ kaolinite (Parra et al., 1986). Relative abundances of these two minerals, however, change in response to microclimate. On Grenada, for example, kaolinite is the only mineral present in soils exposed to high rainfall, whereas smectite is dominant within regions of lower rainfall (Beaven and Dumbleton, 1966). Some smectite in the DSDP/ODP study area almost certainly was formed as a weathering product from this volcanic source.

Geologic investigations of the Lesser Antilles arc show that volcanism has been sporatic in response to reorganizations of the plate boundary (Bouysse et al., 1990). The early Miocene time period corresponding to the lithologic boundary between Units II and III is particularly noteworthy. Evidently, the northern part of the arc became dormant from $\sim 31$ to $22 \mathrm{Ma}$, and a similar hiatus affected the southern part of the arc from between 30 and $28 \mathrm{Ma}$ to $19 \mathrm{Ma}$ (Bouysse et al., 1990). The lithologic boundary separates strata with late early Miocene microfossils, and the nannofossil-bearing turbidites below the lithologic boundary (Subunit IIIb), which are late Oligocene in age (Shipboard Scientific Party, 1995a). Incorporation of abundant volcanic ash into the sediments during a lengthy volcanic hiatus would be difficult to explain. The gradual increase in $\%$ smectite across the décollement zone, however, seems to coincide with gradual renewal of eruptive activity within the arc during the 22-19 Ma time period.

Variations in the respective contributions of smectite, illite, and kaolinite during middle Tertiary time also must have been influenced by eustatic sea-level cycles. For example, Quaternary turbidites in the southern part of the accretionary prism were derived mostly from the Orinoco River during the last glacial lowstand, whereas clay-mineral assemblages in Holocene sediments (higher in smectite) indicate a source on the Amazon and Guyana shelf and margin, with northwesterly transport by surface currents (Faugères et al., 1991). Volumetri- 


\begin{tabular}{|c|c|c|c|c|c|c|c|c|c|c|c|c|c|c|c|c|c|c|c|c|c|}
\hline \multirow{3}{*}{$\begin{array}{l}\text { Core, section, } \\
\text { interval }(\mathrm{cm})\end{array}$} & \multirow{3}{*}{$\begin{array}{l}\text { Depth } \\
\text { (mbsf) }\end{array}$} & \multirow{3}{*}{$\begin{array}{l}\text { Sample } \\
\text { code }\end{array}$} & \multirow{3}{*}{$\begin{array}{r}\text { Strat. } \\
\text { unit }\end{array}$} & \multicolumn{6}{|c|}{4 to $8-\mu \mathrm{m}$ size fraction } & & & to $4-\mu \mathrm{m}$ siz & fractio & & & & & $<1-\mu \mathrm{m}$ size & action & & \\
\hline & & & & Integrated & peak are & $\mathrm{a}$ (counts) & Estin & ed per & ntage & Integrated & peak are & $\mathrm{a}$ (counts) & Esti1 & ted per & entage & Integrated & peak are & ea (counts) & Estim & ted per & ntage \\
\hline & & & & Smectite & Illite & Kaolinite & $\% \mathrm{~S}$ & $\% \mathrm{I}$ & $\% \mathrm{~K}$ & Smectite & Illite & Kaolinite & $\% \mathrm{~S}$ & $\% \mathrm{I}$ & $\% \mathrm{~K}$ & Smectite & Illite & Kaolinite & $\% \mathrm{~S}$ & $\% \mathrm{I}$ & $\% \mathrm{~K}$ \\
\hline 156-949A- & & & & & & & & & & & & & & & & & & & & & \\
\hline $\begin{array}{l}1 \mathrm{H}-1,102-107 \\
1 \mathrm{H}-1,143-150\end{array}$ & $\begin{array}{l}1.02 \\
1.43\end{array}$ & $\begin{array}{l}\text { UND } \\
\text { IW }\end{array}$ & $\begin{array}{l}\text { I } \\
\text { I }\end{array}$ & 6,351 & 2,116 & 3,723 & 29 & 38 & 33 & 7,931 & 2,671 & 4,030 & 30 & 40 & 30 & $\begin{array}{l}8,468 \\
3,272\end{array}$ & $\begin{array}{l}3,538 \\
1,659\end{array}$ & $\begin{array}{l}6,819 \\
2,993\end{array}$ & $\begin{array}{l}23 \\
21\end{array}$ & $\begin{array}{l}39 \\
42\end{array}$ & $\begin{array}{l}38 \\
38\end{array}$ \\
\hline 156-949B- & & & & & & & & & & & & & & & & & & & & & \\
\hline $1 \mathrm{X}-1,87-90$ & 244.97 & UND & $\mathrm{IIb}$ & 8,380 & 1,212 & 4,096 & 39 & 23 & 38 & 13,469 & 2,153 & 5,611 & 40 & 26 & 34 & 28,526 & 2,193 & 7,923 & 54 & 17 & 30 \\
\hline $1 \mathrm{X}-1,120-150$ & 245.30 & IW & $\mathrm{IIb}$ & & & & & & & & & & & & & 14,216 & 998 & 3,553 & 56 & 16 & 28 \\
\hline $2 \mathrm{X}-1,28-42$ & 254.08 & AM & $\mathrm{IIb}$ & 2,017 & 1,106 & 2,247 & 18 & 40 & 41 & 6,224 & 2,152 & 4,428 & 26 & 36 & 37 & 23,648 & 7,887 & 11,432 & 30 & 40 & 29 \\
\hline $2 X-1,60-74$ & 254.40 & AM & $\mathrm{IIb}$ & 2,838 & 418 & 1,161 & $\begin{array}{l}10 \\
42\end{array}$ & 24 & 34 & 8,708 & 1,119 & $\begin{array}{l}2,381 \\
2,381\end{array}$ & 49 & 25 & 27 & 28,418 & $\begin{array}{l}1,001 \\
1,396\end{array}$ & 6,345 & 61 & 12 & 27 \\
\hline $2 \mathrm{X}-2,72-88$ & 256.02 & WB & III & $\begin{array}{l}2,050 \\
2,987\end{array}$ & $\begin{array}{r}410 \\
1,429\end{array}$ & 3,493 & 19 & 36 & 45 & 2,523 & 695 & $\begin{array}{r}2,001 \\
935\end{array}$ & 35 & 39 & 26 & $\begin{array}{l}20,410 \\
17,525\end{array}$ & $\begin{array}{l}1,390 \\
6,572\end{array}$ & $\begin{array}{r}0,543 \\
15,411\end{array}$ & $\begin{array}{l}01 \\
23\end{array}$ & 35 & 41 \\
\hline $2 \mathrm{X}-2,101-106$ & 256.31 & UND & IIc & 4,588 & 800 & 2,021 & 39 & 27 & 34 & 8,001 & 1,105 & 3,356 & 42 & 23 & 35 & 30,490 & 2,410 & 7,626 & 55 & 17 & 28 \\
\hline $2 \mathrm{X}-3,0-40$ & 256.80 & MK & IIc & 4,500 & ovo & 2,021 & & 21 & & 0,001 & 1,105 & ס & & 25 & & 20,350 & 3,060 & 9,083 & 40 & 24 & 36 \\
\hline $2 \mathrm{X}-5,35-40$ & 260.15 & UND & IIc & 5,759 & 1,005 & 2,520 & 39 & 27 & 34 & 6,282 & 1,563 & 3,086 & 34 & 33 & 33 & 33,865 & 2,024 & 7,822 & 59 & 14 & 27 \\
\hline $2 \mathrm{X}-5,110-150$ & 260.90 & IW & III & & & & & & & & & & & & & 27,256 & 4,801 & 11,768 & 39 & 27 & 34 \\
\hline $2 \mathrm{X}-6,10-25$ & 255.51 & GZ & IIc & 5,196 & 1,505 & 3,403 & 29 & 33 & 38 & 7,238 & 2,634 & 6,651 & 23 & 34 & 43 & 19,388 & 3,642 & 12,052 & 33 & 25 & 42 \\
\hline $3 \mathrm{X}-2,24-29$ & 265.24 & UND & III & 7,093 & 1,134 & 2,326 & 44 & 28 & 29 & 13,084 & 2,248 & 7,745 & 35 & 24 & 41 & 39,741 & 2,484 & 15,331 & 49 & 12 & 38 \\
\hline $3 \mathrm{X}-2,87-101$ & 265.87 & $\mathrm{AF}$ & III & 4,171 & 890 & 1,120 & 42 & 36 & 22 & 3,936 & 1,166 & 1,727 & 33 & 39 & 29 & 19,417 & 3,102 & 10,372 & 37 & 24 & 39 \\
\hline $3 \mathrm{X}-4,16-32$ & 268.16 & HT & IIc & 4,160 & 993 & 1,344 & 38 & 37 & 25 & 10,701 & 1,446 & 2,934 & 48 & 26 & 26 & 22,239 & 1,527 & 2,456 & 67 & 18 & 15 \\
\hline $3 \mathrm{X}-5,110-150$ & 270.60 & IW & IIc & & & & & & & & & & & & & 16,154 & 2,351 & 7,165 & 40 & 24 & 36 \\
\hline $3 \mathrm{X}-6,58-63$ & 271.58 & UND & IIc & 5,058 & 1,188 & 1,187 & 42 & 39 & 19 & 14,718 & 1,394 & 3,680 & 53 & 20 & 27 & 27,745 & 1,690 & 5,010 & 62 & 15 & 23 \\
\hline $4 \mathrm{X}-1,106-108$ & 274.16 & UND & IIc & 5,284 & $\begin{array}{l}1,100 \\
1,233\end{array}$ & $\begin{array}{l}1,101 \\
2,374\end{array}$ & 35 & 33 & 32 & $\begin{array}{r}5,783 \\
5,710\end{array}$ & 2,707 & 6,073 & 20 & 38 & 42 & 28,417 & $\begin{array}{l}1,090 \\
2,368\end{array}$ & 6,410 & 56 & 19 & 25 \\
\hline $4 \mathrm{X}-1,125-150$ & 274.35 & IW & III & J,204 & 1,253 & 2,014 & 53 & 53 & 52 & 3,103 & $2, / 0 /$ & 0,075 & 20 & 30 & 42 & $\begin{array}{l}20,411 \\
33,139\end{array}$ & $\begin{array}{l}2,500 \\
3,461\end{array}$ & $\begin{array}{l}0,410 \\
7,219\end{array}$ & 54 & 23 & 24 \\
\hline $5 \mathrm{X}-1,94-110$ & 283.74 & WB & IIc & 3,900 & 3,298 & 6,306 & 13 & 44 & 42 & 4,116 & 860 & 2,734 & 32 & 26 & 42 & 34,381 & 2,002 & 5,160 & 65 & 15 & 20 \\
\hline $5 \mathrm{X}-1,144-148$ & 284.24 & UND & IIc & 6,525 & 1,060 & 1,400 & 48 & 31 & 21 & 5,566 & 828 & 1,014 & 51 & 30 & 19 & 31,172 & 1,299 & 1,326 & 80 & 13 & 7 \\
\hline $5 \mathrm{X}-2,110-150$ & 285.40 & MK & IIc & & & & & & & & & & & & & 24,037 & 1,248 & 3,449 & 67 & 14 & 19 \\
\hline $5 \mathrm{X}-4,43-56$ & 287.73 & JA & IIc & 5,230 & 1,186 & 1,579 & 40 & 36 & 24 & 18,356 & 2,563 & 5,943 & 45 & 25 & 29 & 33,910 & 1,417 & 4,721 & 69 & 12 & 19 \\
\hline $5 X-4,70-73$ & 288.00 & UND & IIc & 5,052 & 1,194 & 2,320 & 35 & 33 & 32 & 14,218 & 2,547 & 5,982 & 39 & 28 & 33 & 32,003 & $\begin{array}{l}1,761 \\
1,761\end{array}$ & $\begin{array}{l}4,169 \\
6,169\end{array}$ & 62 & 14 & 24 \\
\hline $5 \mathrm{X}-4,110-150$ & 288.40 & IW & IIc & & & & & & & & & & & & & 32,528 & 2,379 & 4,657 & 63 & 19 & 18 \\
\hline $5 \mathrm{X}-6,16-19$ & 290.46 & UND & IIc & 4,680 & 611 & 759 & 54 & 28 & 18 & & & & & & & 28,053 & 1,260 & 2,389 & 74 & 13 & 13 \\
\hline $5 X-6,78-94$ & 291.08 & GZ & IIc & 3,716 & 734 & 879 & 44 & 35 & 21 & 4,577 & 997 & 1,005 & 43 & 38 & 19 & 22,714 & 2,039 & 2,891 & 62 & 22 & 16 \\
\hline $5 \mathrm{X}-6,114-130$ & 291.44 & $\mathrm{HT}$ & IIc & 4,631 & 682 & 899 & 51 & 30 & 20 & 9,951 & 1.662 & 2,910 & 44 & 30 & 26 & 17,463 & 1,175 & 2,305 & 65 & 18 & 17 \\
\hline $7 \mathrm{X}-2,12-19$ & 303.72 & UND & $\mathrm{IIb}$ & 5,407 & 1,932 & 1,343 & 34 & 49 & 17 & 6,966 & 2,164 & 3,329 & 31 & 39 & 30 & 19,937 & 4,384 & 6,851 & 39 & 34 & 27 \\
\hline $7 \mathrm{X}-2,110-150$ & 304.70 & IW & $\mathrm{IIb}$ & I & 1,902 & 1,545 & 34 & 49 & 17 & 0,900 & 2,104 & S, & 31 & 39 & 50 & 12,113 & $\begin{array}{l}4,504 \\
4,695\end{array}$ & $\begin{array}{l}0,031 \\
8,328\end{array}$ & 25 & 39 & 35 \\
\hline $7 \mathrm{X}-3,14-25$ & 305.24 & $\mathrm{PH}$ & $\mathrm{IIb}$ & 4,192 & 1,383 & 1.851 & 31 & 41 & 28 & 3,848 & 2,007 & 3,462 & 20 & 43 & 37 & $\begin{array}{l}12,115 \\
15,477\end{array}$ & $\begin{array}{l}4,095 \\
3.275\end{array}$ & $\begin{array}{r}0,520 \\
10,152\end{array}$ & 32 & 27 & 42 \\
\hline $7 \mathrm{X}-4,42-55$ & 307.02 & JA & IIb & $\begin{array}{l}4,192 \\
1.518\end{array}$ & $\begin{array}{l}1,503 \\
1.199\end{array}$ & $\begin{array}{l}1,031 \\
1.287\end{array}$ & 17 & 年4 & 29 & 3.997 & 2,883 & $\begin{array}{l}3,402 \\
2.161\end{array}$ & 25 & $\begin{array}{l}43 \\
48\end{array}$ & 27 & 8.511 & 2,477 & 6.902 & 26 & 31 & 43 \\
\hline $7 \mathrm{X}-4,84-88$ & 307.44 & UND & III & 5,666 & $\begin{array}{l}1,199 \\
1,524\end{array}$ & $\begin{array}{l}1,281 \\
2,599\end{array}$ & 33 & $\begin{array}{l}34 \\
36\end{array}$ & 31 & 7,507 & $\begin{array}{l}1,083 \\
2,018\end{array}$ & $\begin{array}{l}2,101 \\
2,551\end{array}$ & 36 & $\begin{array}{l}48 \\
39\end{array}$ & 25 & $\begin{array}{r}8,011 \\
11,882\end{array}$ & $\begin{array}{l}2,471 \\
4,501\end{array}$ & $\begin{array}{l}0,902 \\
8,838\end{array}$ & $\begin{array}{l}20 \\
25\end{array}$ & 38 & 37 \\
\hline $\begin{array}{l}7 \mathrm{X}-4,84-88 \\
7 \mathrm{X}-4,119-135\end{array}$ & $\begin{array}{l}301.44 \\
307.99\end{array}$ & $\begin{array}{l}\text { UND } \\
\text { HT }\end{array}$ & $\begin{array}{l}11 \mathrm{Ib} \\
\mathrm{IIb}\end{array}$ & $\begin{array}{l}3,606 \\
3,235\end{array}$ & $\begin{array}{l}1,524 \\
1,641\end{array}$ & $\begin{array}{l}2,599 \\
1,977\end{array}$ & $\begin{array}{l}33 \\
24\end{array}$ & $\begin{array}{l}56 \\
48\end{array}$ & $\begin{array}{l}31 \\
29\end{array}$ & $\begin{array}{l}4,5016 \\
4,916\end{array}$ & $\begin{array}{l}2,018 \\
2,825\end{array}$ & $\begin{array}{l}2,531 \\
4,040\end{array}$ & $\begin{array}{l}36 \\
20\end{array}$ & $\begin{array}{l}39 \\
47\end{array}$ & 33 & $\begin{array}{r}11,882 \\
7,799\end{array}$ & $\begin{array}{l}4,301 \\
3,863\end{array}$ & $\begin{array}{l}8,838 \\
7,324\end{array}$ & 21 & $\begin{array}{l}38 \\
41\end{array}$ & $\begin{array}{l}31 \\
39\end{array}$ \\
\hline $7 \mathrm{X}-5,118-134$ & 309.28 & GZ & $\mathrm{IIb}$ & 4,828 & 1,865 & 2,703 & 27 & 42 & 31 & 4,736 & 1,749 & 2,816 & 27 & 40 & 32 & 4,498 & 2,524 & 5,462 & 18 & 40 & 43 \\
\hline $7 \mathrm{X}-6,62-67$ & 310.22 & WB & IIb & 3,824 & 2,049 & 2,484 & 23 & 48 & 29 & 5,872 & 3,029 & 4,649 & 22 & 44 & 34 & 9,922 & 3,340 & 8,094 & $\begin{array}{l}10 \\
25\end{array}$ & 34 & 41 \\
\hline $7 \mathrm{X}-6,110-150$ & 310.70 & IW & $\mathrm{IIb}$ & & 2,049 & 2,404 & & & & & 3,029 & & & & & 22,8 & 3,015 & $\begin{array}{l}\quad, 074 \\
5,719\end{array}$ & 49 & 26 & 25 \\
\hline $13 \mathrm{X}-1,50-6$ & 350.70 & $\mathrm{~PB}$ & $\mathrm{IIb}$ & 4,620 & 467 & 1,169 & 52 & 21 & 26 & 3,543 & 992 & 1,568 & 33 & 37 & 29 & 28,418 & 1,396 & 6,345 & 61 & 12 & 27 \\
\hline $13 \mathrm{X}-1,110-150$ & 351.30 & IW & $\mathrm{IIb}$ & & & & & & & & & & & & & 16 & 2,334 & 6,914 & 42 & 24 & 35 \\
\hline $13 \mathrm{X}-3,30-33$ & 353.50 & UND & IIc & 2,679 & 1,802 & 3,848 & 15 & 41 & 44 & 2,679 & 1,802 & 3,848 & 15 & 41 & 44 & & 2,460 & 10,110 & 39 & 20 & 41 \\
\hline $14 \mathrm{X}-1,45-49$ & 355.35 & UND & IIc & & 3,130 & 5,4 & 11 & 17 & i & 4,015 & 2,158 & 3, & 39 & 20 & 41 & 14,739 & 3,517 & 13,595 & 26 & 25 & 49 \\
\hline $14 \mathrm{X}-1,105-121$ & 355.95 & $\mathrm{~PB}$ & IIc & 3,994 & 1,196 & 2,410 & 29 & 35 & 35 & 8,094 & 1,747 & 5,867 & 30 & 26 & 44 & 46,794 & 2,799 & 22,541 & 45 & 11 & 44 \\
\hline $14 \mathrm{X}-2,54-70$ & 356.94 & $\mathrm{GZ}$ & IIc & 3,678 & 1,132 & 1,332 & 34 & 42 & 25 & $\begin{array}{l}6,0733 \\
6,733\end{array}$ & 1,398 & 2,458 & 39 & 32 & 29 & 30,026 & 4,169 & 10,171 & 45 & 25 & 30 \\
\hline $14 \mathrm{X}-4,33-45$ & 359.73 & JA & IIc & 5,490 & 1,373 & 2,435 & 35 & 35 & 31 & 8,061 & 2,514 & $\begin{array}{l}2,430 \\
6,201\end{array}$ & 26 & 33 & 41 & $\begin{array}{l}50,400 \\
27,430\end{array}$ & $\begin{array}{l}4,436 \\
2,436\end{array}$ & $\begin{array}{r}10,171 \\
8,003\end{array}$ & 52 & 18 & 30 \\
\hline $14 \mathrm{X}-4,62-78$ & 360.02 & HT & III & $\begin{array}{l}2,490 \\
2,856\end{array}$ & 737 & $\begin{array}{l}2,433 \\
1,411\end{array}$ & 33 & 34 & 33 & $\begin{array}{r}8,001 \\
20,753\end{array}$ & $\begin{array}{l}2,14 \\
1,656\end{array}$ & $\begin{array}{l}\text {,2,201 } \\
5,764\end{array}$ & $\begin{array}{l}20 \\
53\end{array}$ & 17 & $\begin{array}{l}41 \\
30\end{array}$ & $\begin{array}{l}21,430 \\
25,068\end{array}$ & $\begin{array}{l}2,430 \\
2,330\end{array}$ & $\begin{array}{l}8,003 \\
5,968\end{array}$ & 54 & 20 & 26 \\
\hline $14 \mathrm{X}-5,80-82$ & 361.70 & UND & IIc & 3,110 & 1,377 & 2,389 & 23 & 41 & 36 & 9,271 & 2,410 & 4,902 & 32 & 34 & 34 & 16,909 & 2.481 & 7,554 & 40 & 24 & 36 \\
\hline $14 \mathrm{X}-5,110-150$ & 362.00 & IW & IIc & & & & & & & & & & & & & 36,028 & 2,871 & 11,592 & 51 & 16 & 33 \\
\hline 15 & 361.66 & UND & IIc & 6,293 & 729 & 1,470 & 52 & 24 & 24 & 7,783 & 1,167 & 2,303 & 46 & 27 & 27 & 27,2 & 1,020 & 4,657 & 67 & 10 & 23 \\
\hline $2,110-150$ & 362.50 & IW & IIc & & & & & & & & & & & & & & & & 39 & 27 & 34 \\
\hline $15 \mathrm{X}-3,1$ & 36 & UND & IIc & 4,629 & 1,028 & 2,264 & 35 & 31 & 3 & 8,8 & 2,071 & 4,4 & 34 & 32 & 34 & & & & 55 & 20 & 25 \\
\hline 15 & & $\mathrm{H}$ & IIc & & 1,0 & & 5 & 21 & 2 & $\begin{array}{l}9,0,0 \\
9,0\end{array}$ & 1,5 & 2,9 & 43 & 29 & 2 & & 1, & 2,393 & 56 & 22 & 22 \\
\hline $15 \mathrm{X}-3,122-138$ & 364.12 & $\mathrm{~PB}$ & IIc & 4,1 & 585 & 1,1 & 47 & 26 & 26 & 3,190 & 648 & 585 & 46 & 37 & 17 & 33,500 & 2,235 & 5,601 & 62 & 17 & 21 \\
\hline $15 \mathrm{X}-4,41-57$ & 364.81 & GZ & IIc & 4,399 & 1,532 & 3,921 & 24 & 33 & 43 & 10,438 & 3,000 & 6,228 & 30 & 34 & 36 & 28, & 5,237 & 10,445 & 40 & 30 & 30 \\
\hline $15 \mathrm{X}-5,28-44$ & 366.18 & WB & IIc & 3,821 & 437 & 463 & 59 & 27 & 14 & & & & & & & 24,398 & 1.209 & 2,743 & 70 & 14 & 16 \\
\hline $15 X-5,65-76$ & 366.55 & $\mathrm{PH}$ & IIc & 7,460 & 0 & 0 & 100 & 0 & 0 & 7,189 & 415 & 0 & 81 & 19 & 0 & 27,618 & 0 & 1,652 & 89 & $\begin{array}{r}14 \\
0\end{array}$ & 11 \\
\hline $15 X-5,110-150$ & 367.00 & IW & IIc & & & & & & & 1,109 & 415 & & 01 & 19 & 0 & 25,014 & 2,118 & $\begin{array}{l}1,032 \\
4,490\end{array}$ & $\begin{array}{l}09 \\
59\end{array}$ & 20 & 21 \\
\hline $15 X-6,57-65$ & 367.97 & $\mathrm{SP}$ & IIc & 5,866 & 879 & 1.900 & 45 & 27 & 29 & 8,773 & 1.554 & 3,069 & 42 & 29 & 29 & 25,566 & 965 & 3,889 & 69 & 10 & 21 \\
\hline $15 X-6,119-135$ & 368.59 & WB & IIc & 2,725 & 567 & 1,393 & 35 & 29 & 36 & 3,112 & 536 & 1,008 & 43 & 29 & 28 & 32,115 & 2,044 & 5,329 & 63 & 16 & 21 \\
\hline- CC, $0-6$ & 379.10 & IW & IIc & & & & & & & & & & & & & 31,015 & 2,150 & 10,968 & 50 & 14 & 36 \\
\hline$-1,33-50$ & 399.13 & WB & IIe & 2,547 & 1,288 & 1,756 & 23 & 46 & 31 & 042 & 969 & 667 & 25 & 39 & 36 & 23,148 & 1,630 & 12,130 & 43 & 12 & 45 \\
\hline 3 & 40 & IW & IIe & & & & & & & & & & & & & & 3800 & 11 , & 29 & 28 & 43 \\
\hline $\mathrm{X}-2,32-48$ & 400.62 & $\mathrm{I}$ & Ie & 5,069 & 1974 & 3 & 26 & 41 & 33 & 9770 & 23 & 5464 & 32 & 31 & 31 & 23810 & 2,303 & 8,830 & 47 & 18 & \\
\hline
\end{tabular}


Table 2 (continued).

\begin{tabular}{|c|c|c|c|c|c|c|c|c|c|c|c|c|c|c|c|c|c|c|c|c|c|}
\hline \multirow{3}{*}{$\begin{array}{l}\text { Core, section, } \\
\text { interval }(\mathrm{cm})\end{array}$} & \multirow{3}{*}{$\begin{array}{l}\text { Depth } \\
\text { (mbsf) }\end{array}$} & \multirow{3}{*}{$\begin{array}{l}\text { Sample } \\
\text { code }\end{array}$} & \multirow{3}{*}{$\begin{array}{c}\text { Strat. } \\
\text { unit }\end{array}$} & \multicolumn{6}{|c|}{4 to $8-\mu \mathrm{m}$ size fraction } & \multicolumn{6}{|c|}{ 1-to $4-\mu \mathrm{m}$ size fraction } & \multicolumn{6}{|c|}{$<1-\mu \mathrm{m}$ size fraction } \\
\hline & & & & \multicolumn{3}{|c|}{ Integrated peak area (counts) } & \multicolumn{3}{|c|}{ Estimated percentage } & \multicolumn{3}{|c|}{ Integrated peak area (counts) } & \multicolumn{3}{|c|}{ Estimated percentage } & \multicolumn{3}{|c|}{ Integrated peak area (counts) } & \multicolumn{3}{|c|}{ Estimated percentage } \\
\hline & & & & Smectite & Illite & Kaolinite & $\% \mathrm{~S}$ & $\% \mathrm{I}$ & $\% \mathrm{~K}$ & Smectite & Illite & Kaolinite & $\% \mathrm{~s}$ & $\% \mathrm{I}$ & $\% \mathrm{~K}$ & Smectite & Illite & Kaolinite & $\% \mathrm{~S}$ & $\% \mathrm{I}$ & $\% \mathrm{~K}$ \\
\hline $19 \mathrm{X}-3,10-14$ & 401.40 & UND & IIe & 1,403 & 760 & 1,550 & 19 & 40 & 41 & 5,692 & 1,634 & 3,641 & 29 & 34 & 37 & 26,604 & 2,448 & 10,909 & 46 & 17 & 37 \\
\hline $19 \mathrm{X}-3,34-50$ & 401.64 & GZ & IIe & 2,578 & 500 & 950 & 40 & 31 & 29 & 6,273 & 1,562 & 2,489 & 36 & 36 & 28 & 26,310 & 942 & 8,344 & 56 & 8 & 36 \\
\hline $19 \mathrm{X}-3,95-106$ & 402.25 & JA & IIe & 3,800 & 1,780 & 1,569 & 27 & 51 & 22 & 7,887 & 2,731 & 5,029 & 27 & 38 & 35 & 16,557 & 2,245 & 10,798 & 35 & 19 & 46 \\
\hline $\begin{array}{l}19 \mathrm{X}-4,38-42 \\
\end{array}$ & 403.18 & UND & IIe & 6,913 & 1,354 & 2,040 & 42 & 33 & 25 & 10,591 & 2,343 & 3,485 & 39 & 35 & 26 & 21,376 & 2,651 & 8,288 & 44 & 22 & 34 \\
\hline $22 \mathrm{X}-1,23-27$ & 427.63 & UND & IIIe & $\begin{array}{l}0,817 \\
1,817\end{array}$ & 1,080 & 1,401 & 20 & 48 & 31 & $\begin{array}{r}1,764 \\
6,764\end{array}$ & 2,088 & 3,730 & 30 & 37 & 33 & 27,812 & $\begin{array}{l}2,031 \\
4,512\end{array}$ & $\begin{array}{r}0,200 \\
17,642\end{array}$ & 34 & 22 & 43 \\
\hline $22 \mathrm{X}-1,130-146$ & 428.70 & WB & IIIe & 3,407 & 1,378 & $\begin{array}{l}1,401 \\
2,516\end{array}$ & 24 & $\begin{array}{l}40 \\
40\end{array}$ & $\begin{array}{l}31 \\
36\end{array}$ & 2,908 & 2,904 & 6,392 & 11 & 43 & 47 & 21,690 & 3,555 & 18,518 & 30 & 19 & 51 \\
\hline $22 \mathrm{X}-2,0-16$ & 428.90 & $\mathrm{GZ}$ & $\begin{array}{l}\text { IIIe } \\
\text { IIe }\end{array}$ & $\begin{array}{l}5,4019 \\
1,319\end{array}$ & 1,724 & 2,184 & 10 & $\begin{array}{l}40 \\
55\end{array}$ & $\begin{array}{l}50 \\
35\end{array}$ & $\begin{array}{l}2,968 \\
4,964\end{array}$ & 2,543 & $\begin{array}{l}0,592 \\
4,556\end{array}$ & $\begin{array}{l}11 \\
20\end{array}$ & 42 & 38 & 18,407 & 3,251 & 12,907 & 32 & 23 & 45 \\
\hline $22 \mathrm{X}-3,1-13$ & 430.42 & SP & IIe & 3,440 & 1,556 & 2,442 & 24 & 43 & 34 & 5,518 & 1,903 & 3,596 & 27 & 37 & 35 & 27,111 & 2,349 & 10,423 & 47 & 16 & 36 \\
\hline $22 \mathrm{X}-4,31-35$ & 432.21 & UND & III & 3,771 & 1,375 & 1,563 & 30 & 44 & 25 & 8,426 & 2,899 & 3,995 & 30 & 41 & 29 & 27,652 & 4,689 & 15,153 & 36 & 24 & 40 \\
\hline $22 \mathrm{X}-4,38-54$ & 432.28 & HT & III & 5,050 & 2,530 & 2,841 & 24 & 49 & 27 & 8,426 & 2,899 & 3,995 & 30 & 41 & 29 & 28,659 & 4,770 & 12,026 & 40 & 27 & 34 \\
\hline $22 \mathrm{X}-4,121-125$ & 433.11 & UND & III & $\begin{array}{l}6,729 \\
6,030\end{array}$ & 2,068 & 3,464 & 31 & 38 & 32 & $\begin{array}{l}0,420 \\
7,935\end{array}$ & 2,536 & 4,767 & 29 & $\begin{array}{l}41 \\
37\end{array}$ & 35 & 20,580 & 4,294 & 11,580 & 34 & 28 & 38 \\
\hline $22 \mathrm{X}-5,110-150$ & 434.50 & IW & III & & & & & & & & & & & & & 19,911 & 3,635 & 7,340 & 41 & 30 & 30 \\
\hline $22 \mathrm{X}-6,137-140$ & 436.27 & UND & III & 5,241 & 1,949 & 4,346 & 24 & 36 & 40 & 8,059 & 3,235 & 6,290 & 24 & 39 & 37 & 34,702 & 8,343 & 13,490 & 37 & 35 & 28 \\
\hline $25 \mathrm{H}-1,92-96$ & 459.32 & UND & III & 2,104 & 1,286 & 2,023 & 19 & 46 & 36 & 5,330 & 3,499 & 7,055 & 16 & 42 & 42 & 9,272 & 7,241 & 20,083 & 12 & 37 & 51 \\
\hline $25 \mathrm{H}-2,9-13$ & 459.99 & UND & III & 2,553 & 1.536 & 3,627 & 16 & 39 & 45 & 3,642 & 3,091 & 4,739 & 14 & 49 & 37 & 8,400 & 12,688 & 22,394 & 8 & 49 & 43 \\
\hline $25 \mathrm{H}-2,61-65$ & 460.51 & UND & III & 3,068 & 729 & 864 & 40 & 38 & 22 & 3,109 & 712 & 2,038 & $\begin{array}{l}31 \\
31\end{array}$ & 28 & 41 & 18,613 & 2,044 & 31,311 & 21 & 9 & 70 \\
\hline $25 \mathrm{H}-2,84-93$ & 460.74 & JA & III & $\begin{array}{l}1,667 \\
1,607\end{array}$ & 1,585 & 3,998 & 10 & $\begin{array}{l}50 \\
40\end{array}$ & 50 & 1,584 & 4,756 & 12,345 & $\begin{array}{r}1 \\
3\end{array}$ & $\begin{array}{l}20 \\
42\end{array}$ & $\begin{array}{l}41 \\
55\end{array}$ & $\begin{array}{r}10,256 \\
6,256\end{array}$ & 7,385 & 21,444 & $\begin{array}{r}1 \\
8\end{array}$ & 38 & 55 \\
\hline $25 \mathrm{H}-3,110-150$ & 462.50 & IW & III & & & & & & & & & & & & & 11,265 & 8,469 & 20,526 & 13 & 39 & 48 \\
\hline \multicolumn{22}{|l|}{$156-949 \mathrm{C}-$} \\
\hline 2R-CC, $5-10$ & 405.95 & IW & IIe & & & & & & & & & & & & & 25,836 & 1,432 & 3,370 & 67 & 15 & 18 \\
\hline $4 \mathrm{R}-1,0-5$ & 425.20 & IW & III & & & & & & & & & & & & & 18,470 & 3,380 & 14,587 & 30 & 22 & 48 \\
\hline $7 \mathrm{R}-1,80-90$ & 454.90 & IW & III & & & & & & & & & & & & & 7,380 & 5,661 & 12,915 & 13 & 41 & 46 \\
\hline
\end{tabular}

Note: See Table 1 for explanation of sample codes. 


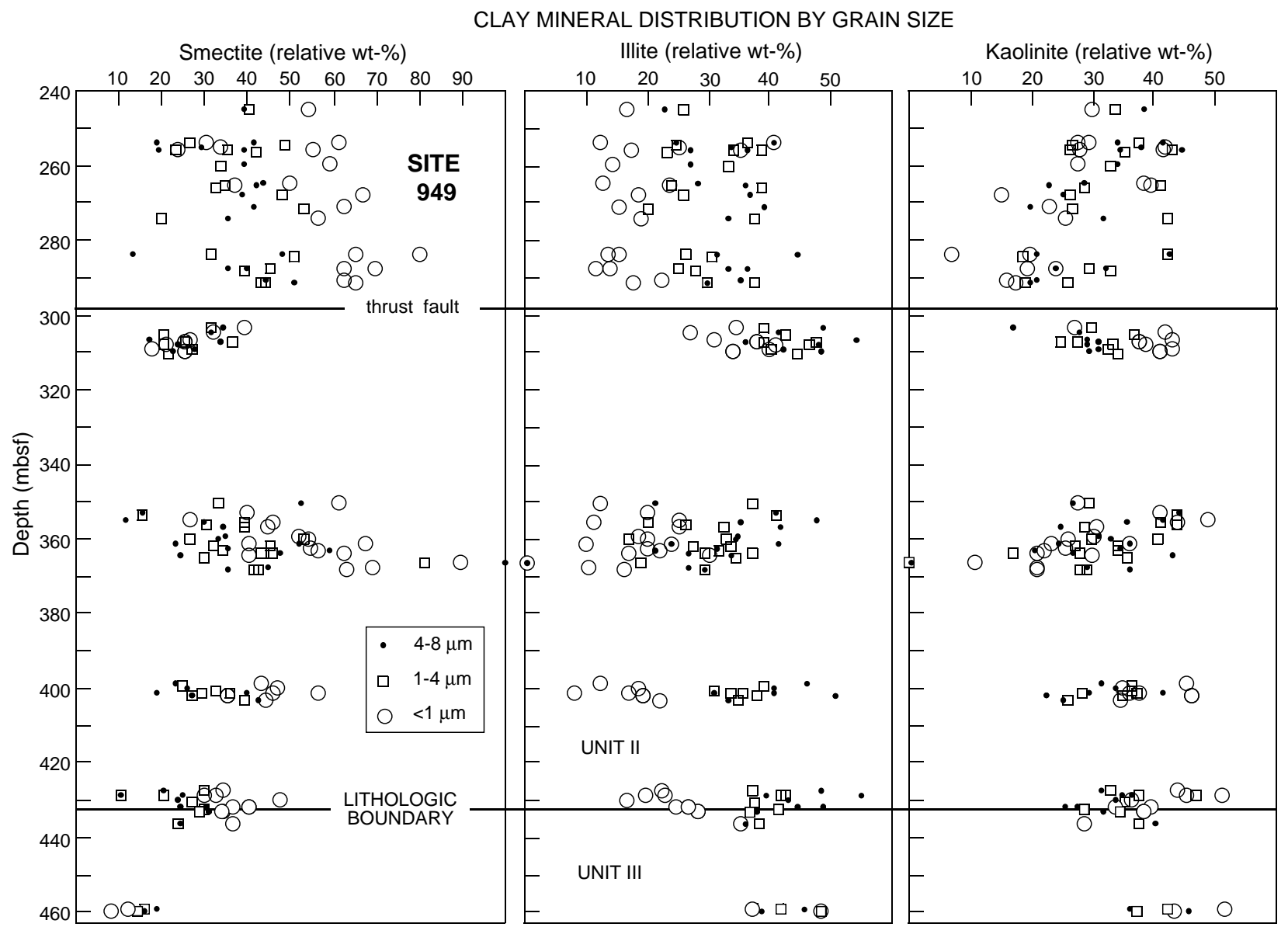

Figure 10. Partitioning of clay minerals as a function of grain size, lithofacies Units II and III, Site 949.

cally, the delivery of South American detritus into deep-water sites likewise changed in response to Quaternary sea-level fluctuations, but those effects diminished with increasing distance from the continental margin. Detrital flux of volcanogenic smectite into the northern portion of the Lesser Antilles forearc and the adjacent Atlantic Abyssal Plain seemingly has decreased during the Holocene highstand, based on relatively low percentages of expandable clay minerals in surface and near-surface sediments (Wright, 1984; Reid et al., 1996). Consequently, we suspect that remobilization of smectite-rich soils within the Lesser Antilles arc probably increased during each of the Cenozoic lowstands as larger parts of the arc platform became exposed to subaerial weathering and soil erosion. Biostratigraphic resolution for Sites 671 and 948 is nowhere near what is required for detailed analysis of potential eustatic cycles in sediment delivery, but we note that sea levels were significantly lower during late Oligocene time as compared to the late early Miocene (Haq et al., 1988). Thus, a rise in sea level across the time interval spanning the lithologic boundary should have caused a decrease in the transport of arc-derived detrital smectite relative to the aeolian influx of volcanic ash.

The Lesser Antilles arc may be the most obvious source of detrital smectite, but several studies showed that South American fluvial systems have transported considerable amounts of expandable clay toward the Caribbean Basin and the Atlantic during late Quaternary time. Relative percentages of clay minerals in the back-arc Venezuela Basin, for example, average $42 \%$ smectite, $31 \%$ kaolinite, and $27 \%$ illite (Parra et al., 1986). Basin and canyon deposits from the southern part of the Barbados accretionary prism average $20 \%$ smectite, $45 \%$ kaolinite, and 30\% illite (Faugères et al., 1991). Evidently, these near-surface sediments were transported initially from the Orinoco and Amazon river mouths before their redeposition by turbidity currents. According to Faugères et al. (1993), the average composition of Orinoco-derived shelf sediment is 38\% kaolinite, $27 \%$ illite, $20 \%$ smectite, and $15 \%$ chlorite. The smectite content of Quaternary muds near the mouth of the Amazon River ranges from 35\% up to $75 \%$, and this substantial increase in expandable clay provides a reliable criterion for discriminating between Amazon and Orinoco discharge. Seaward of the deformation front of Barbados Ridge, sandy turbidite deposits on the Atlantic abyssal floor contain the following clay-mineral percentages: $30 \%-40 \%$ illite, $20 \%-35 \%$ kaolinite, and 15\%-30\% smectite; most of those near-surface sediments were transported from an Orinoco River source during the last glacial lowstand (Faugères et al., 1993).

Collectively, models of late Quaternary sediment dispersal show that clay-mineral abundances, by themselves, are inadequate for isolating the respective volumetric contributions of smectite-rich clays from the Lesser Antilles, the proto-Orinoco, and the proto-Amazon River sources during Oligocene-Miocene time. On the other hand, we know that South American sources contributed significantly to the Oligocene-Miocene sediment budget. TEM images of the Oligocene-Miocene samples, for example, display clear evidence for incorporation of detrital chlorite and the $2 \mathrm{M}$ polytype of illite/mica (Buatier et al., 1992). Those minerals must have come from South American sources (based on their total absence in Quaternary discharge from the volcanic arc), and it is logical to assume that the detrital chlorite and illite particles were transported together with some detrital smectite. As added support for a South American source, Tribble (1990) showed that anomalously coarse-grained Eocene turbidite deposits at Site 672 (Dolan et al., 1990) also contain unusually 


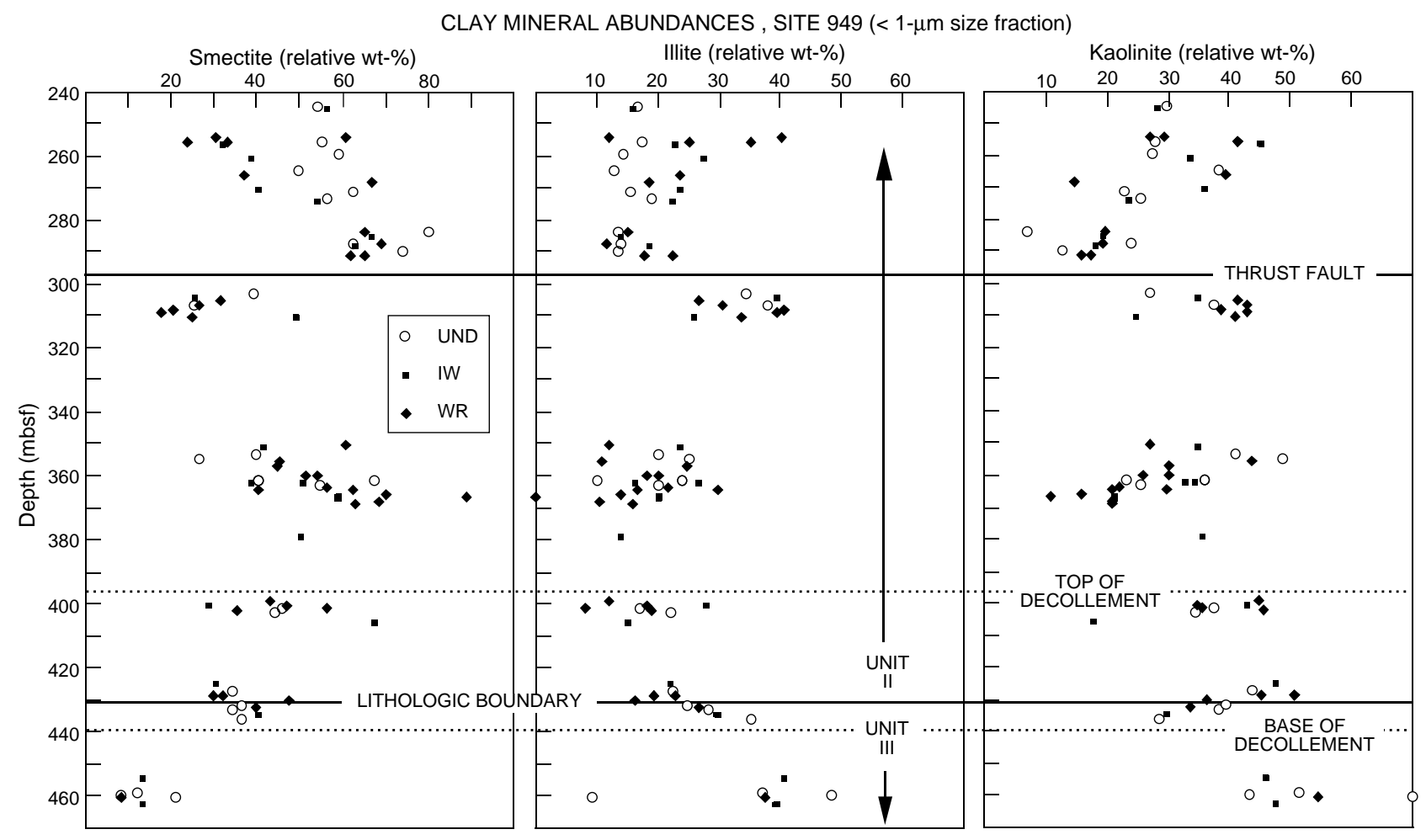

Figure 11. Individual depth profiles of relative abundance for smectite, illite, and kaolinite in clay-sized fraction $(<1 \mu \mathrm{m})$ of samples from Site 949 . See Table 2 for specific sample numbers, depths, integrated peak areas, and clay-mineral percentages based on Biscaye (1965) peak-area weighting factors.

high percentages of smectite $(32 \%-67 \%)$. Coeval quartz-rich sandstones from the island of Barbados have been linked unequivocally to specific continental source areas in South America through apatite fission-track fingerprints (Baldwin et al., 1986; Kasper and Larue, 1986). We infer that interbeds of smectite-rich mudstone within the Eocene turbidite succession were derived largely from the same continental weathering processes. In conclusion, detrital smectite from South America has been an important, though variable, part of the regional sediment budget throughout Cenozoic time.

\section{Smectite Composition}

SEM/EDS analyses of bulk clay-sized aggregates from Site 948 show that consistent correlations exist between estimated percentages of smectite and most of the major oxides (Fig. 15). Correlations are particularly robust for $\mathrm{SiO}_{2} / \mathrm{Al}_{2} \mathrm{O}_{3}$ and $\mathrm{Fe}_{\text {total }} / \mathrm{Al}_{2} \mathrm{O}_{3}$ vs. \% smectite. Kaolinite also generates a clear chemical signal, but statistically significant correlations are lacking between values of $\%$ illite and oxide weight percentages. It is important to note, however, that $\mathrm{K}_{2} \mathrm{O}$ content tends to remain relatively high in all of the specimens analyzed, even those with high values of $\%$ smectite and low values of $\%$ illite. These data also have bearing on interpretations regarding the origin of smectite-rich clays and I/S mixed-layer phases.

Previous chemical analyses of samples from Sites 671 and 672 demonstrated that the smectitic clays in question are actually quite variable in composition. For example, the mineral formulas calculated by Capet et al. (1990) (i.e., numbers of cations) range from $\mathrm{K}_{0.01}$ to $\mathrm{K}_{0.33}, \mathrm{Mg}_{0.12}$ to $\mathrm{Mg}_{0.62}$, and $\mathrm{Fe}_{0.18}$ to $\mathrm{Fe}_{0.98}$. The highest values for $\mathrm{K}$ and $\mathrm{Fe}$ are similar to those expected for end-member nontronite, and most of the specimens are probably best regarded as Al-Fe-beidellites with variable amounts of octahedral substitution. Buatier et al. (1992) obtained more precise AEM data from two aggregates of matrix smectite $( \pm \mathrm{I} / \mathrm{S})$ located just above the décollement zone at Site 671 . Their results show that the $\mathrm{K}_{2} \mathrm{O}$ contents of the smectite-rich compos- ites range from 0.62 to $3.27 \mathrm{wt} \% ; \mathrm{Fe}_{2} \mathrm{O}_{3}$ varies between 15.73 and $4.40 \mathrm{wt} \%$, and $\mathrm{MgO}$ is $1.28-5.27 \mathrm{wt} \%$. Calculated numbers of cations in the corresponding chemical formulas are $\mathrm{K}_{0.11}-\mathrm{K}_{0.57}, \mathrm{Fe}_{0.45}-$ $\mathrm{Fe}_{1.70}$, and $\mathrm{Mg}_{0.27}-\mathrm{Mg}_{1.06}$. Buatier et al. (1992) described this smectite matrix as "very homogeneous in composition." In our opinion, however, without even considering the specific source(s), the variations in multiple chemical components are large enough to indicate that smectite-rich clays were not derived from a single source or a single process of in situ alteration of a chemically homogeneous volcanic precursor.

Parra et al. (1986) showed that chemical characteristics of South American smectites in Quaternary sediments differ markedly from those eroded from the Lesser Antilles. Their results are unambiguous and may provide the best criterion for defining smectite provenance. Expandable clays in Quaternary marine sediments fringing the volcanic islands consist of $\mathrm{Fe}$-montmorillonite and $\mathrm{Fe}$-Al-beidellite rich in $\mathrm{MgO}(1.63-4.48 \mathrm{wt} \%)$ and poor in $\mathrm{K}_{2} \mathrm{O}(0.20-0.23 \mathrm{wt} \%)$. The South American variety of smectite, in contrast, is an Fe-Al-beidellite rich in $\mathrm{K}_{2} \mathrm{O}(2.51 \mathrm{wt} \%)$ and poor in $\mathrm{MgO}(1.79 \mathrm{wt} \%)$. The samples from our study are older and may have been modified by burial diagenesis, but they obviously show a closer geochemical affinity to the smectite from South America.

As a final argument, Chamley (1989) stressed that Al-Fe-beidellite is the most abundant clay mineral within most claystones of late Mesozoic and early Cenozoic age throughout the west-central Atlantic Basin; volcanic glass is rare within these samples. Theoretically, all of that smectite could have formed exclusively by complete in situ replacement of volcanic ash, but such a scenario would require unreasonably large amounts of persistent explosive volcanism and persistent discharge of ash into the Atlantic by aeolian processes.

We believe that South American drainage basins represent the most likely primary source for the background level of smectite delivery into the Barbados Ridge depositional system during Oligocene-Miocene time. Sharp increases above this background were 
Table 3. X-ray diffraction data for illite/smectite mixed-layer clay phases from mudstones and claystones at Sites 948 and 949.

\begin{tabular}{|c|c|c|c|c|}
\hline \multirow[b]{3}{*}{$\begin{array}{l}\text { Core, section, } \\
\text { interval }(\mathrm{cm})\end{array}$} & \multirow[b]{3}{*}{$\begin{array}{l}\text { Depth } \\
\text { (mbsf) }\end{array}$} & \multicolumn{3}{|c|}{$\%$ illite in I/S mixed-layer clay } \\
\hline & & \multicolumn{2}{|c|}{$\begin{array}{c}(001 / 002)-(002 / 003) \\
\text { two-peak method }\end{array}$} & \multirow{2}{*}{$\begin{array}{c}\begin{array}{c}\text { Saddle/(001) } \\
\text { method }\end{array} \\
<1-\mu \mathrm{m} \\
\text { fraction }\end{array}$} \\
\hline & & $\begin{array}{l}<0.2-\mu \mathrm{m} \\
\text { fraction }\end{array}$ & $\begin{array}{l}<1-\mu \mathrm{m} \\
\text { fraction }\end{array}$ & \\
\hline \multicolumn{5}{|l|}{ 156-948A- } \\
\hline $2 X-3,64-67$ & 424.44 & 15 & 18 & 23 \\
\hline $3 \mathrm{X}-2,20-24$ & 432.20 & & 21 & \\
\hline $3 X-6,88-92$ & 438.88 & 34 & 26 & \\
\hline $4 \mathrm{X}-4,55-58$ & 445.15 & 29 & 28 & 32 \\
\hline $4 X-6,49-52$ & 449.09 & & 17 & \\
\hline $5 X-2,85-89$ & 452.15 & & 16 & \\
\hline $5 X-4,39-43$ & 454.69 & 21 & 37 & 28 \\
\hline $6 \mathrm{X}-1,115-119$ & 460.55 & & 39 & 28 \\
\hline $6 \mathrm{X}-2,57-60$ & 461.47 & 29 & 25 & 33 \\
\hline $6 X-3,76-80$ & 463.16 & & 23 & 23 \\
\hline $7 X-4,115-118$ & 474.65 & & 17 & 10 \\
\hline $7 X-6,41-44$ & 476.91 & & 15 & 6 \\
\hline $8 X-1,56-58$ & 479.26 & & 13 & 10 \\
\hline $8 X-4,34-36$ & 483.54 & 16 & 14 & 10 \\
\hline $8 X-7,6-8$ & 487.76 & & 12 & 14 \\
\hline $9 X-1,42-46$ & 488.82 & 23 & 16 & 11 \\
\hline $9 \mathrm{X}-2,26-30$ & 490.16 & 19 & 15 & 12 \\
\hline $9 X-4,63-67$ & 493.53 & 17 & 10 & 12 \\
\hline $10 X-1,121-124$ & 499.31 & 15 & 9 & 9 \\
\hline $10 \mathrm{X}-2,94-98$ & 500.54 & 11 & 22 & 14 \\
\hline $10 X-3,59-62$ & 501.69 & 13 & 20 & 14 \\
\hline $10 X-4,126-130$ & 503.86 & 12 & 20 & 11 \\
\hline $10 X-5,53-56$ & 504.63 & 11 & 14 & 9 \\
\hline $11 X-1,66-70$ & 508.36 & 7 & 13 & 12 \\
\hline $11 X-4,43-47$ & 512.63 & 29 & 19 & 28 \\
\hline $11 X-5,13-17$ & 513.83 & 19 & 36 & 24 \\
\hline $12 X-2,32-33$ & 519.22 & 34 & & 27 \\
\hline $12 X-4,75-78$ & 522.65 & 41 & 41 & 28 \\
\hline $13 X-3,123-126$ & 531.03 & 56 & 35 & 41 \\
\hline $13 \mathrm{X}-4,16-19$ & 531.46 & & 38 & \\
\hline $13 \mathrm{X}-6,21-25$ & 534.51 & & & 29 \\
\hline $14 X-1,45-49$ & 536.55 & 47 & & \\
\hline $14 X-4,26-30$ & 540.86 & & 42 & 31 \\
\hline $14 \mathrm{X}-4,122-126$ & 541.82 & & 32 & 25 \\
\hline $15 X-1,61-65$ & 546.01 & & & 30 \\
\hline $15 X-5,75-79$ & 552.15 & 41 & & \\
\hline $15 X-6,45-49$ & 553.35 & & 29 & 35 \\
\hline $16 X-6,32-36$ & 562.62 & 32 & 40 & 33 \\
\hline $17 X-2,97-99$ & 566.57 & & 43 & 35 \\
\hline $17 X-6,133-135$ & 572.93 & 30 & 27 & 29 \\
\hline $18 \mathrm{X}-2,23-25$ & 575.03 & & 32 & 23 \\
\hline $18 X-4,33-38$ & 577.90 & & 31 & \\
\hline $19 X-2,26-30$ & 584.56 & & 38 & 16 \\
\hline $19 X-4,56-58$ & 587.86 & & 39 & 11 \\
\hline $19 X-7,40-43$ & 592.20 & 36 & 25 & 7 \\
\hline
\end{tabular}

\begin{tabular}{|c|c|c|c|c|}
\hline \multirow[b]{3}{*}{$\begin{array}{l}\text { Core, section, } \\
\text { interval }(\mathrm{cm})\end{array}$} & \multirow[b]{3}{*}{$\begin{array}{l}\text { Depth } \\
\text { (mbsf) }\end{array}$} & \multicolumn{3}{|c|}{$\%$ illite in $\mathrm{I} / \mathrm{S}$ mixed-layer clay } \\
\hline & & \multicolumn{2}{|c|}{$\begin{array}{c}(001 / 002)-(002 / 003) \\
\text { two-peak method }\end{array}$} & \multirow{2}{*}{$\begin{array}{c}\text { Saddle/(001) } \\
\text { method }\end{array}$} \\
\hline & & $\begin{array}{l}<0.2-\mu \mathrm{m} \\
\text { fraction }\end{array}$ & $\begin{array}{l}<1-\mu \mathrm{m} \\
\text { fraction }\end{array}$ & \\
\hline \multicolumn{5}{|l|}{ 156-949B- } \\
\hline $1 \mathrm{X}-1,87-90$ & 244.97 & & 17 & 28 \\
\hline $2 \mathrm{X}-1,60-74$ & 254.40 & & 14 & 17 \\
\hline $2 \mathrm{X}-2,101-106$ & 256.31 & & & 25 \\
\hline $2 \mathrm{X}-5,25-40$ & 260.15 & & & 27 \\
\hline $3 \mathrm{X}-6,58-63$ & 271.58 & & & 19 \\
\hline $4 \mathrm{X}-1,106-108$ & 274.16 & & & 25 \\
\hline $5 \mathrm{X}-1,144-148$ & 284.24 & & & 16 \\
\hline $5 X-4,43-56$ & 287.73 & & 12 & \\
\hline $5 X-4,70-73$ & 288.00 & & & 23 \\
\hline $5 \mathrm{X}-4,110-150$ & 288.40 & & 8 & \\
\hline $5 X-6,16-19$ & 290.46 & & & 17 \\
\hline $7 X-4,42-55$ & 307.02 & & 24 & \\
\hline $7 \mathrm{X}-5,118-134$ & 309.28 & & 27 & \\
\hline $13 \mathrm{X}-1,50-66$ & 350.70 & & 34 & 9 \\
\hline $13 \mathrm{X}-3,30-33$ & 353.50 & & & 29 \\
\hline $14 \mathrm{X}-1,45-49$ & 355.35 & & & 29 \\
\hline $14 \mathrm{X}-1,105-121$ & 356.00 & & 25 & \\
\hline $14 X-2,54-70$ & 356.94 & & 37 & \\
\hline $14 \mathrm{X}-5,80-82$ & 361.70 & & & 25 \\
\hline $15 X-1,144-146$ & 361.34 & & 17 & \\
\hline $15 \mathrm{X}-2,26-30$ & 361.66 & & 19 & 14 \\
\hline $15 \mathrm{X}-3,19-23$ & 363.09 & & & 19 \\
\hline $15 X-3,84-100$ & 363.74 & & 21 & \\
\hline $15 \mathrm{X}-4,41-57$ & 364.81 & & 25 & \\
\hline $15 X-5,28-44$ & 366.18 & & 40 & 3 \\
\hline $15 X-5,65-76$ & 366.55 & & 22 & \\
\hline $15 X-6,57-65$ & 367.97 & & 14 & \\
\hline $15 \mathrm{X}-6,119-135$ & 368.59 & & 16 & \\
\hline $19 \mathrm{X}-1,33-50$ & 399.13 & & 23 & \\
\hline $19 \mathrm{X}-2,32-48$ & 400.62 & & 35 & \\
\hline $19 \mathrm{X}-3,10-14$ & 401.40 & & 19 & 12 \\
\hline $19 \mathrm{X}-3,34-50$ & 401.64 & & 21 & 17 \\
\hline $19 \mathrm{X}-4,38-42$ & 403.18 & & 21 & 11 \\
\hline $22 \mathrm{X}-1,23-27$ & 427.63 & & 25 & 25 \\
\hline $22 \mathrm{X}-1,130-146$ & 428.70 & & 32 & \\
\hline $22 \mathrm{X}-2,0-16$ & 428.90 & & 47 & \\
\hline $22 X-2,110-150$ & 429.90 & & 25 & \\
\hline $22 \mathrm{X}-3,2-13$ & 430.42 & & 33 & \\
\hline $22 \mathrm{X}-4,31-35$ & 432.21 & & 18 & 29 \\
\hline $22 \mathrm{X}-4,121-125$ & 433.11 & & 29 & 27 \\
\hline $22 \mathrm{X}-6,137-140$ & 436.27 & & 39 & \\
\hline $25 \mathrm{H}-1,92-96$ & 459.32 & & 32 & \\
\hline $25 \mathrm{H}-2,61-65$ & 460.51 & & 38 & 36 \\
\hline
\end{tabular}

Notes: Peaks for $\Delta^{\circ} 2 \theta$ method are illustrated in Figure 4. See Inoue et al. (1989) for description of saddle/(001) intensity ratio and Table 1 for explanation of sample codes.
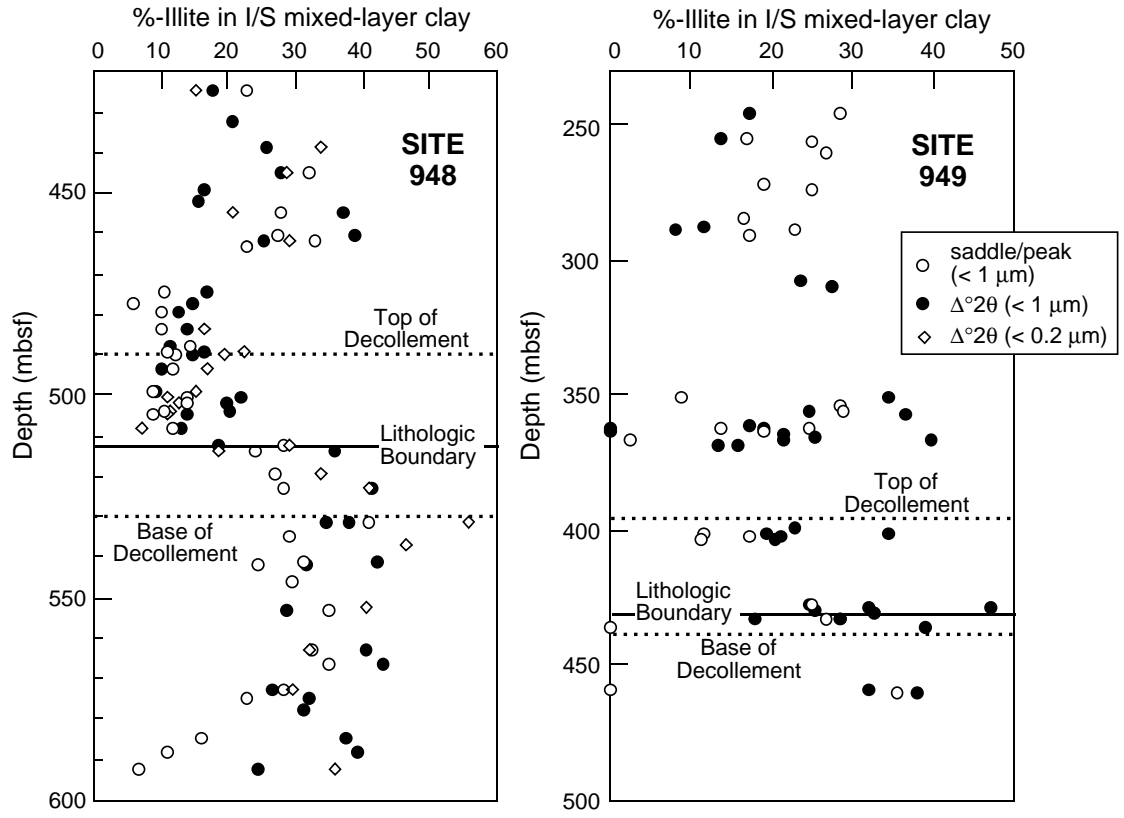

Figure 12. Estimates of $\%$ illite in I/S mixed-layer clays from Sites 948 and 949 based on angular separation of the $\mathrm{S}(002) / \mathrm{I}(001)$ and $\mathrm{S}(003) / \mathrm{I}(002)$ peaks (see Fig. 4) and the saddle/(001) method (see Inoue et al., 1989). See Table 3 for sample identification and individual values. 
Table 4. Major-oxide concentrations and oxide ratios in samples from Site 948.

\begin{tabular}{|c|c|c|c|c|c|c|c|c|c|c|c|c|c|c|c|c|c|}
\hline \multirow{2}{*}{$\begin{array}{l}\text { Core, section, } \\
\text { interval }(\mathrm{cm})\end{array}$} & \multirow{2}{*}{$\begin{array}{l}\text { Depth } \\
\text { (mbsf) }\end{array}$} & \multicolumn{3}{|c|}{ Clay mineral percentage } & \multicolumn{7}{|c|}{ Weight percentages of major oxides } & \multicolumn{6}{|c|}{ Ratios of major oxides } \\
\hline & & $\% \mathrm{~S}$ & $\% \mathrm{I}$ & $\% \mathrm{~K}$ & $\mathrm{Al}_{2} \mathrm{O}_{3}$ & $\mathrm{SiO}_{2}$ & $\mathrm{~K}_{2} \mathrm{O}$ & $\mathrm{Fe}_{\text {total }}$ & $\mathrm{MgO}$ & $\mathrm{CaO}$ & $\mathrm{TiO}_{2}$ & $\mathrm{Si} / \mathrm{Al}$ & $\mathrm{K} / \mathrm{Al}$ & $\mathrm{Fe} / \mathrm{Al}$ & $\mathrm{Mg} / \mathrm{Al}$ & $\mathrm{Ca} / \mathrm{Al}$ & $\mathrm{Ti} / \mathrm{Al}$ \\
\hline \multicolumn{18}{|l|}{ Unit II } \\
\hline \multicolumn{18}{|l|}{$156-948 \mathrm{C}-$} \\
\hline $\begin{array}{l}2 X-3,64-67 \\
2 X-948\end{array}$ & 424.44 & 53 & 27 & 20 & 17.66 & 50.92 & 2.01 & 11.88 & 3.69 & 0.92 & 0.73 & 2.88 & 0.114 & 0.672 & 0.209 & 0.052 & 0.0416 \\
\hline $\begin{array}{l}2 \mathrm{X}-3,64-6 / \\
2 \mathrm{X}-6,41-46\end{array}$ & $\begin{array}{l}424.44 \\
428.71\end{array}$ & $\begin{array}{l}53 \\
44\end{array}$ & 31 & 25 & $\begin{array}{l}17.66 \\
16.57\end{array}$ & $\begin{array}{l}50.92 \\
48.77\end{array}$ & 2.07 & $\begin{array}{l}11.88 \\
11.78\end{array}$ & $\begin{array}{l}3.69 \\
2.00\end{array}$ & $\begin{array}{l}0.92 \\
0.99\end{array}$ & 0.74 & $\begin{array}{l}2.88 \\
2.94\end{array}$ & $\begin{array}{l}0.114 \\
0.125\end{array}$ & $\begin{array}{l}0.711 \\
0.712\end{array}$ & $\begin{array}{l}0.209 \\
0.121\end{array}$ & $\begin{array}{l}0.052 \\
0.060\end{array}$ & $\begin{array}{l}0.0416 \\
0.0448\end{array}$ \\
\hline $4 \mathrm{X}-2,114-117$ & 442.72 & 36 & 26 & 38 & 18.03 & 47.73 & 1.88 & 11.98 & 1.76 & 0.97 & 0.72 & 2.65 & 0.104 & 0.664 & 0.097 & 0.054 & 0.0398 \\
\hline $4 \mathrm{X}-4,55-58$ & 445.15 & 31 & 24 & 45 & 19.50 & 47.09 & 2.20 & 11.87 & 2.04 & 1.58 & 0.61 & 2.41 & 0.113 & $\begin{array}{l}0.609 \\
0.609\end{array}$ & 0.105 & 0.081 & 0.0312 \\
\hline $4 X-6,49-52$ & 449.08 & 52 & 23 & 25 & 19.38 & 50.04 & 1.47 & 8.81 & 3.80 & 1.07 & 0.59 & 2.58 & 0.076 & 0.455 & 0.196 & 0.055 & 0.0305 \\
\hline $5 X-2,85-89$ & 452.15 & 49 & 17 & 34 & 19.52 & 50.25 & 1.68 & 9.54 & 3.66 & 1.22 & 0.64 & 2.57 & 0.086 & 0.489 & 0.188 & 0.062 & 0.0329 \\
\hline $5 \mathrm{X}-4,39-43$ & 454.69 & 43 & 22 & 35 & 16.49 & 45.43 & 1.66 & 10.18 & 1.00 & 3.79 & 0.72 & 2.75 & 0.101 & 0.617 & 0.060 & 0.230 & 0.0435 \\
\hline $6 \mathrm{X}-1,115-119$ & 460.55 & 47 & 15 & 38 & 18.76 & 48.17 & 1.81 & 9.46 & 2.08 & 1.64 & 0.55 & 2.57 & 0.097 & 0.504 & 0.111 & 0.088 & 0.0293 \\
\hline $6 \mathrm{X}-2,57-60$ & 461.47 & 28 & 18 & 54 & 21.10 & 47.25 & 1.96 & 12.13 & 2.05 & 0.74 & 0.60 & 2.24 & 0.093 & 0.575 & 0.097 & 0.035 & 0.0284 \\
\hline $6 \mathrm{X}-3,76-80$ & 463.16 & 53 & 15 & 32 & 17.43 & 47.32 & 1.74 & 11.06 & 0.80 & 0.84 & 0.66 & 2.72 & 0.100 & 0.635 & 0.046 & 0.048 & 0.0378 \\
\hline $7 \mathrm{X}-4,115-118$ & 474.65 & 71 & 12 & 17 & 17.62 & 49.11 & 1.31 & $\begin{array}{l}11.00 \\
11.88\end{array}$ & $\begin{array}{l}0.00 \\
3.42\end{array}$ & $\begin{array}{l}.04 \\
1.18\end{array}$ & $\begin{array}{l}0.00 \\
0.61\end{array}$ & 2.79 & $\begin{array}{l}0.074 \\
0.074\end{array}$ & 0.674 & $\begin{array}{l}.0 .040 \\
0.194\end{array}$ & $\begin{array}{l}0.040 \\
0.067\end{array}$ & 0.0346 \\
\hline $8 \mathrm{X}-1,56-58$ & 479.26 & 70 & 13 & 17 & 16.49 & 49.85 & 1.31 & $\begin{array}{l}11.80 \\
11.22\end{array}$ & $\begin{array}{l}3.42 \\
3.38\end{array}$ & $\begin{array}{l}1.18 \\
1.42\end{array}$ & $\begin{array}{l}0.01 \\
0.95\end{array}$ & 3.02 & $\begin{array}{l}0.074 \\
0.080\end{array}$ & $\begin{array}{l}0.074 \\
0.680\end{array}$ & $\begin{array}{l}0.194 \\
0.205\end{array}$ & $\begin{array}{l}0.007 \\
0.086\end{array}$ & $\begin{array}{l}0.0340 \\
0.0577\end{array}$ \\
\hline $\begin{array}{l}8 X-1,50-58 \\
8 X-4,34-36\end{array}$ & $\begin{array}{l}483.50 \\
483.54\end{array}$ & 76 & $\begin{array}{l}13 \\
10\end{array}$ & 14 & $\begin{array}{l}10.49 \\
15.88\end{array}$ & $\begin{array}{l}49.83 \\
50.62\end{array}$ & $\begin{array}{l}1.31 \\
0.95\end{array}$ & $\begin{array}{l}11.22 \\
10.74\end{array}$ & $\begin{array}{l}3.38 \\
4.21\end{array}$ & $\begin{array}{l}1.42 \\
1.45\end{array}$ & $\begin{array}{l}0.95 \\
1.14\end{array}$ & $\begin{array}{l}3.02 \\
3.19\end{array}$ & $\begin{array}{l}0.080 \\
0.060\end{array}$ & $\begin{array}{l}0.080 \\
0.676\end{array}$ & 0.265 & $\begin{array}{l}0.086 \\
0.091\end{array}$ & 0.0720 \\
\hline $\begin{array}{l}8 X-4,43-36 \\
* 8 X-4,34-36\end{array}$ & $\begin{array}{l}48.54 \\
483.54\end{array}$ & $\begin{array}{l}16 \\
76\end{array}$ & $\begin{array}{l}10 \\
10\end{array}$ & $\begin{array}{l}14 \\
14\end{array}$ & $\begin{array}{l}15.88 \\
15.73\end{array}$ & $\begin{array}{l}50.62 \\
50.47\end{array}$ & $\begin{array}{l}0.93 \\
1.02\end{array}$ & $\begin{array}{l}10.74 \\
10.64\end{array}$ & $\begin{array}{l}4.21 \\
3.98\end{array}$ & $\begin{array}{l}1.45 \\
1.58\end{array}$ & $\begin{array}{l}1.14 \\
0.93\end{array}$ & $\begin{array}{l}3.19 \\
3.21\end{array}$ & $\begin{array}{l}0.060 \\
0.065\end{array}$ & $\begin{array}{l}0.676 \\
0.677\end{array}$ & $\begin{array}{l}0.263 \\
0.253\end{array}$ & $\begin{array}{l}0.091 \\
0.101\end{array}$ & $\begin{array}{l}0.0720 \\
0.0589\end{array}$ \\
\hline $9 X-1,42-46$ & $\begin{array}{l}485.54 \\
488.82\end{array}$ & $\begin{array}{l}10 \\
64\end{array}$ & 14 & $\begin{array}{l}14 \\
22\end{array}$ & 16.97 & 50.82 & $\begin{array}{l}1.02 \\
1.12\end{array}$ & $\begin{array}{l}10.04 \\
11.46\end{array}$ & $\begin{array}{l}3.98 \\
3.81\end{array}$ & $\begin{array}{l}1.58 \\
1.60\end{array}$ & $\begin{array}{l}0.93 \\
0.63\end{array}$ & $\begin{array}{l}3.21 \\
2.99\end{array}$ & $\begin{array}{l}0.005 \\
0.066\end{array}$ & 0.675 & 0.225 & $\begin{array}{l}0.101 \\
0.094\end{array}$ & 0.0369 \\
\hline $9 \mathrm{X}-2,26-30$ & 490.16 & $\begin{array}{l}64 \\
62\end{array}$ & $\begin{array}{l}14 \\
15\end{array}$ & 22 & 20.02 & 49.55 & 1.77 & 11.73 & $\begin{array}{l}3.01 \\
1.92\end{array}$ & 0.78 & 0.44 & 2.47 & 0.088 & 0.586 & 0.096 & 0.039 & 0.0221 \\
\hline$* 9 \mathrm{X}-2,26-30$ & 490.16 & 62 & 15 & 22 & $\begin{array}{l}20.77 \\
16.77\end{array}$ & 50.53 & 1.13 & 13.10 & 3.16 & 1.45 & $\begin{array}{l}0.84 \\
0.89\end{array}$ & 3.01 & $\begin{array}{l}0.0067 \\
0.067\end{array}$ & 0.781 & 0.188 & 0.086 & 0.0531 \\
\hline $9 \mathrm{X}-4,63-67$ & 493.53 & 68 & 16 & 16 & 14.86 & 48.16 & 0.90 & 11.71 & 2.61 & 1.04 & 0.81 & 3.24 & $\begin{array}{l}0.001 \\
0.061\end{array}$ & $\begin{array}{l}0.781 \\
0.788\end{array}$ & 0.176 & $\begin{array}{l}0.080 \\
0.070\end{array}$ & 0.0545 \\
\hline $10 \mathrm{X}-1,121-124$ & 499.31 & 51 & 15 & 34 & 16.21 & 48.55 & 1.46 & 10.80 & 2.10 & 1.08 & 0.55 & 3.00 & 0.090 & 0.666 & 0.129 & 0.066 & 0.0339 \\
\hline $10 \mathrm{X}-2,94-98$ & 500.54 & 52 & 20 & 28 & 17.47 & 50.80 & 1.01 & 12.11 & 3.37 & 0.94 & 0.78 & 2.91 & 0.058 & 0.693 & 0.193 & 0.054 & 0.0449 \\
\hline *10X-2, 94-98 & 500.54 & 52 & 20 & 28 & 17.94 & 50.38 & 1.11 & 11.80 & 3.37 & 1.20 & 0.45 & 2.81 & 0.062 & 0.658 & 0.188 & 0.067 & 0.0251 \\
\hline $10 \mathrm{X}-3,59-62$ & 501.69 & 41 & 21 & 39 & 17.44 & 48.73 & 1.13 & 11.43 & 0.79 & 0.84 & 0.70 & 2.79 & 0.065 & 0.655 & 0.045 & 0.048 & 0.0402 \\
\hline $10 \mathrm{X}-4,126-130$ & 503.86 & $\begin{array}{l}71 \\
69\end{array}$ & 14 & 16 & 16.93 & 51.26 & 0.91 & $\begin{array}{l}11.45 \\
10.53\end{array}$ & 4.34 & $\begin{array}{l}.04 \\
1.34\end{array}$ & 0.61 & 3.03 & 0.054 & 0.622 & 0.257 & $\begin{array}{l}0.040 \\
0.079\end{array}$ & 0.0360 \\
\hline $11 \mathrm{X}-4,43-47$ & 512.63 & 35 & $\begin{array}{l}14 \\
22\end{array}$ & $\begin{array}{l}10 \\
43\end{array}$ & $\begin{array}{l}10.93 \\
20.83\end{array}$ & $\begin{array}{l}31.20 \\
48.62\end{array}$ & $\begin{array}{l}0.91 \\
1.68\end{array}$ & 12.73 & $\begin{array}{l}4.34 \\
2.14\end{array}$ & $\begin{array}{l}1.34 \\
0.57\end{array}$ & $\begin{array}{l}.01 \\
0.59\end{array}$ & 2.33 & $\begin{array}{l}0.034 \\
0.081\end{array}$ & $\begin{array}{l}0.022 \\
0.611\end{array}$ & 0.103 & 0.027 & 0.0284 \\
\hline \multirow{2}{*}{\multicolumn{5}{|c|}{$\begin{array}{l}\text { 11X-5, 13-17 } \\
\text { Mean values for Unit II }\end{array}$}} & 20.02 & 49.55 & 1.77 & 11.73 & $\begin{array}{l}2.14 \\
1.92\end{array}$ & 0.78 & 0.44 & 2.47 & $\begin{array}{l}0.081 \\
0.088\end{array}$ & 0.586 & 0.096 & 0.039 & 0.0284 \\
\hline & & & & & 17.82 & 49.20 & 1.48 & 11.29 & 2.70 & 1.24 & 0.68 & 2.78 & & 0.083 & 0.638 & 0.154 & 0.071 \\
\hline \multirow{2}{*}{\multicolumn{18}{|c|}{ Unit III }} \\
\hline & & & & & & & & & & & & & & & & & \\
\hline $12 X-2,32-33$ & 519.22 & 39 & 25 & 36 & 18.75 & 45.54 & 2.12 & 9.03 & 1.43 & 0.60 & 0.72 & 2.43 & 0.113 & 0.482 & 0.076 & 0.032 & 0.0382 \\
\hline $13 \mathrm{X}-3,123-126$ & 531.03 & 29 & 25 & 45 & 21.88 & 48.27 & 2.13 & 11.04 & 2.03 & 0.61 & 0.59 & 2.21 & 0.097 & 0.504 & 0.093 & 0.028 & 0.0271 \\
\hline $13 \mathrm{X}-4,16-19$ & 531.46 & 18 & 26 & 56 & 21.79 & 43.37 & 1.96 & 7.79 & 1.70 & 1.18 & 0.53 & 1.99 & 0.090 & 0.357 & 0.078 & 0.054 & 0.0245 \\
\hline $13 \mathrm{X}-1,21-25$ & 534.51 & 14 & 25 & 61 & 22.37 & 41.36 & 1.05 & 7.38 & 1.67 & 5.97 & 0.77 & 1.85 & 0.047 & 0.330 & 0.074 & 0.267 & 0.0343 \\
\hline $14 \mathrm{X}-1,45-49$ & 536.55 & 19 & 10 & 72 & 24.17 & 47.90 & 2.92 & 8.70 & $\begin{array}{l}1.07 \\
1.78\end{array}$ & 0.61 & 0.53 & $\begin{array}{l}1.03 \\
1.98\end{array}$ & $\begin{array}{l}0.041 \\
0.121\end{array}$ & 0.360 & 0.074 & 0.025 & 0.0221 \\
\hline $14 \mathrm{X}-4,26-30$ & 540.86 & 8 & 46 & 46 & 19.65 & 44.11 & 1.81 & $\begin{array}{r}0.00 \\
12.07\end{array}$ & 2.19 & $\begin{array}{l}0.01 \\
5.52\end{array}$ & 0.35 & $\begin{array}{l}1.98 \\
2.25\end{array}$ & $\begin{array}{l}0.121 \\
0.092\end{array}$ & $\begin{array}{l}0.000 \\
0.614\end{array}$ & 0.111 & 0.281 & 0.0178 \\
\hline $\begin{array}{l}14-4,20-30 \\
* 14 X-4,26-30\end{array}$ & $\begin{array}{l}340.80 \\
540.86\end{array}$ & $\begin{array}{l}8 \\
8\end{array}$ & $\begin{array}{l}40 \\
46\end{array}$ & $\begin{array}{l}40 \\
46\end{array}$ & $\begin{array}{l}19.03 \\
19.86\end{array}$ & 45.07 & $\begin{array}{l}1.81 \\
2.02\end{array}$ & 12.17 & 2.11 & 6.07 & 0.63 & 2.27 & $\begin{array}{l}0.092 \\
0.102\end{array}$ & $\begin{array}{l}0.014 \\
0.613\end{array}$ & 0.106 & $\begin{array}{l}0.281 \\
0.305\end{array}$ & 0.0315 \\
\hline $14 \mathrm{X}-4,122-126$ & $\begin{array}{l}54.80 \\
541.82\end{array}$ & $\begin{array}{r}8 \\
24\end{array}$ & $\begin{array}{l}40 \\
26\end{array}$ & $\begin{array}{l}40 \\
50\end{array}$ & $\begin{array}{l}19.80 \\
23.11\end{array}$ & 41.90 & $\begin{array}{l}2.02 \\
0.96\end{array}$ & $\begin{array}{r}12.17 \\
7.48\end{array}$ & $\begin{array}{l}2.11 \\
1.24\end{array}$ & $\begin{array}{l}0.07 \\
5.28\end{array}$ & $\begin{array}{l}0.03 \\
0.44\end{array}$ & 1.81 & $\begin{array}{l}0.102 \\
0.042\end{array}$ & $\begin{array}{l}0.013 \\
0.324\end{array}$ & $\begin{array}{l}0.100 \\
0.054\end{array}$ & $\begin{array}{l}0.305 \\
0.228\end{array}$ & 0.0191 \\
\hline $\begin{array}{l}14 X-4,122-126 \\
* 14 X-4,122-126\end{array}$ & $\begin{array}{l}541.82 \\
541.82\end{array}$ & 24 & $\begin{array}{l}26 \\
26\end{array}$ & $\begin{array}{l}50 \\
50\end{array}$ & $\begin{array}{l}23.41 \\
24.41\end{array}$ & $\begin{array}{l}41.90 \\
43.60\end{array}$ & $\begin{array}{l}0.96 \\
0.92\end{array}$ & $\begin{array}{l}7.48 \\
7.34\end{array}$ & $\begin{array}{l}1.24 \\
1.52\end{array}$ & $\begin{array}{l}5.28 \\
5.88\end{array}$ & $\begin{array}{l}0.44 \\
0.95\end{array}$ & $\begin{array}{l}1.81 \\
1.79\end{array}$ & $\begin{array}{l}0.042 \\
0.038\end{array}$ & $\begin{array}{l}0.324 \\
0.301\end{array}$ & $\begin{array}{l}0.054 \\
0.062\end{array}$ & $\begin{array}{l}0.228 \\
0.241\end{array}$ & $\begin{array}{l}0.0191 \\
0.0390\end{array}$ \\
\hline $15 \mathrm{X}-1,61-65$ & 546.01 & 13 & 9 & 78 & 21.61 & 48.13 & 1.80 & 10.11 & 2.40 & 0.83 & 0.88 & 2.23 & 0.083 & 0.468 & 0.111 & 0.039 & 0.0409 \\
\hline $15 X-5,45-49$ & 552.15 & 18 & 18 & 64 & 21.22 & 49.50 & $\begin{array}{l}1.00 \\
2.68\end{array}$ & 12.85 & 2.04 & 0.60 & 0.46 & 2.33 & 0.126 & $\begin{array}{l}0.4005 \\
0.605\end{array}$ & 0.096 & 0.028 & 0.0216 \\
\hline $15 \mathrm{X}-6,45-49$ & 553.35 & 17 & 34 & 49 & 20.51 & 47.70 & $\begin{array}{l}2.00 \\
1.90\end{array}$ & 8.83 & 1.29 & 2.38 & 0.54 & 2.33 & 0.093 & 0.431 & 0.063 & 0.116 & 0.0264 \\
\hline $\begin{array}{l}15 X-1,45-49 \\
16 \mathrm{X}-6,32-36\end{array}$ & 562.62 & 24 & 34 & $\begin{array}{l}49 \\
42\end{array}$ & 21.36 & 46.23 & $\begin{array}{l}1.90 \\
2.35\end{array}$ & $\begin{array}{l}.0 .03 \\
7.70\end{array}$ & $\begin{array}{l}1.29 \\
1.97\end{array}$ & $\begin{array}{l}2.38 \\
0.69\end{array}$ & $\begin{array}{l}0.54 \\
0.52\end{array}$ & 2.17 & $\begin{array}{l}0.093 \\
0.110\end{array}$ & $\begin{array}{l}0.431 \\
0.361\end{array}$ & $\begin{array}{l}.0003 \\
0.092\end{array}$ & $\begin{array}{l}0.032 \\
0.032\end{array}$ & 0.0242 \\
\hline $17 \mathrm{X}-2,97-99$ & 566.57 & 22 & 30 & 48 & 21.56 & 48.21 & 2.09 & 9.92 & 2.40 & 2.68 & 0.51 & 2.24 & 0.097 & 0.460 & 0.111 & 0.124 & 0.0236 \\
\hline $17 \mathrm{X}-6,48-53$ & 572.08 & 23 & 15 & 62 & 20.61 & 38.29 & 0.87 & 7.15 & 1.74 & 13.75 & 0.57 & 1.86 & 0.042 & 0.347 & 0.084 & 0.667 & 0.0275 \\
\hline $18 \mathrm{X}-4,33-38$ & 577.90 & 28 & 27 & 45 & 20.55 & 48.67 & 1.80 & 10.35 & 2.26 & 0.76 & 0.39 & 2.37 & 0.088 & 0.503 & 0.110 & 0.037 & 0.0191 \\
\hline $19 \mathrm{X}-2,26-30$ & 584.56 & 28 & 23 & 50 & 21.65 & 46.04 & 1.63 & 8.87 & 1.96 & 6.33 & 0.67 & 2.13 & 0.075 & 0.410 & 0.090 & 0.292 & 0.0308 \\
\hline \multirow{2}{*}{\multicolumn{2}{|c|}{$\begin{array}{l}19 \mathrm{X}-4,56-58 \\
\text { Mean values for Unit III }\end{array}$}} & 30 & 18 & 52 & 22.87 & 50.15 & 2.16 & 10.97 & 2.30 & 0.73 & 0.56 & 2.19 & 0.094 & 0.480 & 0.100 & 0.032 & 0.0244 \\
\hline & & & & & 21.55 & 45.78 & 1.84 & 9.43 & 1.89 & 3.36 & 0.59 & 2.13 & 0.086 & 0.442 & 0.088 & 0.157 & 0.0273 \\
\hline
\end{tabular}

Notes: * = duplicate analysis. Values based on SEM/EDS analyses of oriented aggregates of $<1-\mu \mathrm{m}$ size fractions. 




Figure 13. Depth profiles of major-oxide concentrations in samples from Site 948, based on SEM/EDS analyses of oriented aggregates of $<1-\mu \mathrm{m}$ size fractions. See Table 4 for sample identification and individual weight percentages. 


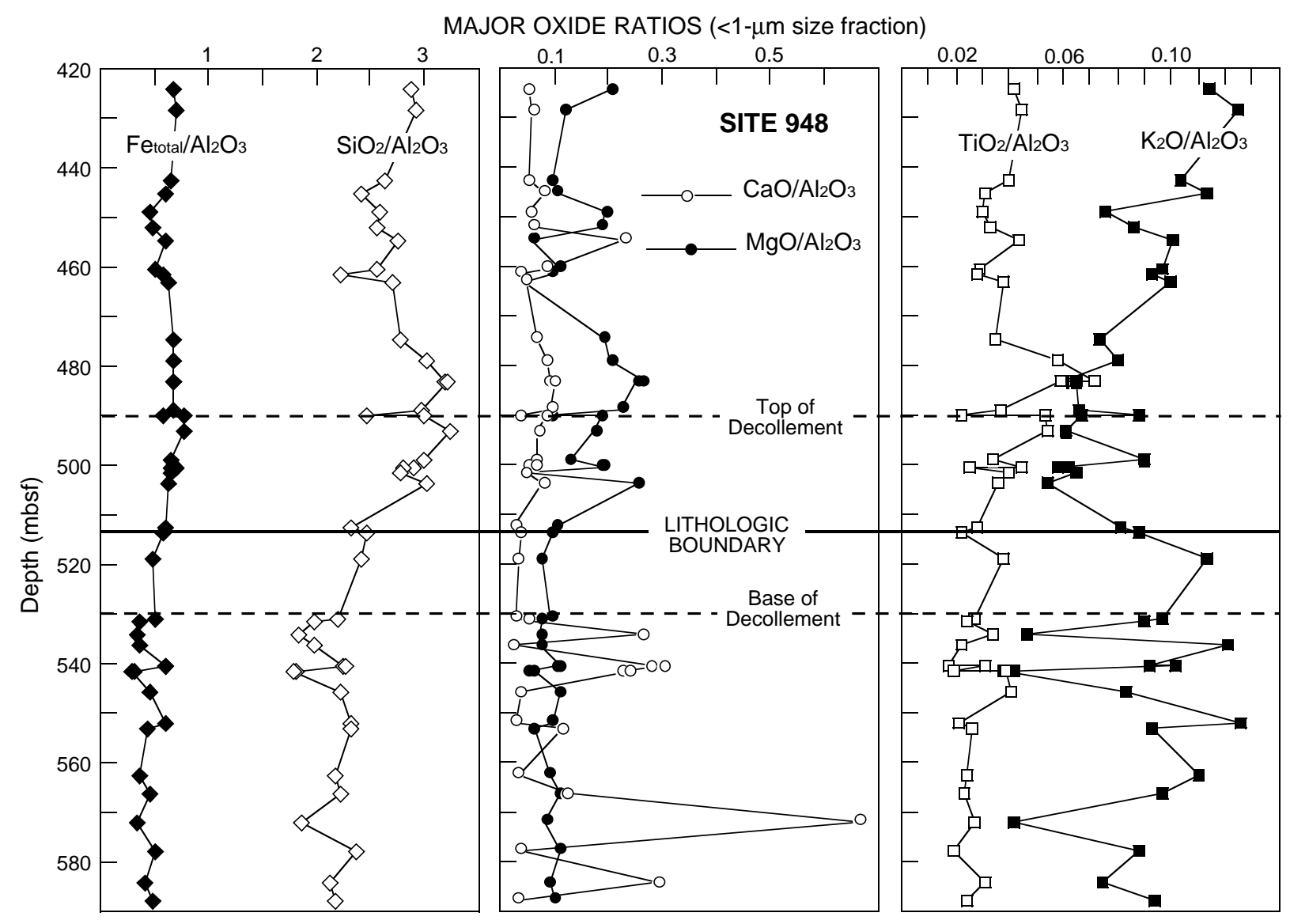

Figure 14. Depth profiles of major-oxide concentrations relative to the concentration of $\mathrm{Al}_{2} \mathrm{O}_{3}$ in samples from Site 948 , based on SEM/EDS analyses of oriented aggregates of $<1-\mu \mathrm{m}$ size fractions. See Table 4 for sample identification and individual ratio values.

caused by transport of additional sediment from the Lesser Antilles volcanic arc. When viewed in total, our results compliment many other lines of evidence (e.g., magnetic susceptibility, bulk-powder XRD mineralogy, abundance of turbidites) and point to a fundamental change in detrital sources at the time corresponding to the lithologic boundary between Units II and III. Within Unit II, we suggest that increases from the background input of $25 \%-55 \%$ smectite to maximum percentages of $55 \%-80 \%$ smectite occurred because of an added component of detrital smectite from the island arc and in situ alteration of disseminated ash from the Lesser Antilles volcanoes. The oxygen isotope data of Tribble and Yeh (1994) show that endmember examples of nearly pure smectite (true bentonites) almost certainly represent altered ash layers, but these deposits are relatively rare.

\section{Evidence of In Situ Illitization at Low Temperatures}

An apparent increase in the illite content of I/S phases below the décollement led Tribble (1990) to conclude that the smectite-to-illite transformation has occurred in situ. This interpretation is provocative because the inferred temperature of illitization near the décollement (500 mbsf) is no greater than about $40^{\circ} \mathrm{C}$, based on the most recent geothermal data from Site 948 (Shipboard Scientific Party, 1995a). Our data from Site 948 show that the I/S clays are more illitic below the boundary between Unit II and Unit III, but we uncovered no evidence in support of illitization within the upper part of the décollement zone (Fig. 12).

Buatier et al. (1992) argued cogently that K-exchange within smectite interlayers is a manifestation of the earliest stage of smec- tite-to-illite transition, although Quaternary smectites from South America also have unusually high contents of $\mathrm{K}_{2} \mathrm{O}$ (Parra et al., 1986). We suspect that the existence of these discrete forms of K-rich smectite as a common matrix constituent is partially responsible for weakening the statistical correlation between $\%$ illite and $\mathrm{K}_{2} \mathrm{O}$ content in the clay-sized aggregates from Site 948 (Fig. 16). The masking effect of abundant detrital illite within Unit III prevents us from confirming with EDS data that a more illitic mixed-layer I/S phase contributes to higher contents of $\mathrm{K}_{2} \mathrm{O}$ in the clay-sized aggregates. On the other hand, the TEM images of Buatier et al. (1992) show that some specimens of smectite from below the décollement contain packets of "neoformed" illite crystallites. The compositional data of Buatier et al. (1992) define a subtle trend of Al-enrichment and K-enrichment with depth, but their data also display considerable scatter within, and overlap among, three specific sample depths. In fact, AEM analyses show that the aggregate $\mathrm{K}_{2} \mathrm{O}$ contents of their deepest clay-sized mixtures $(1.43 \%-3.81 \%)$ fall well within the range established for their discrete smectites above the décollement $(0.62 \%-3.27 \%)$. Chemical evidence, therefore, fails to verify a progressive depth-dependent increase in $\mathrm{K}_{2} \mathrm{O}$ within the clay-sized material, as should be expected with a normal illitization profile (Hower et al., 1976).

Oxygen isotope data are similarly ambiguous with respect to the origin of I/S clays. According to Tribble and Yeh (1994), I/S clays within the underthrust sequence could have formed either by authigenesis under equilibrium conditions with present-day pore waters or by burial diagenesis on the South American continent prior to transport as a detrital phase. Because of the low in situ temperatures near the prism's toe, several previous investigators have suggested that enhanced fluid flow was responsible for illitization (Tribble, 1990; 

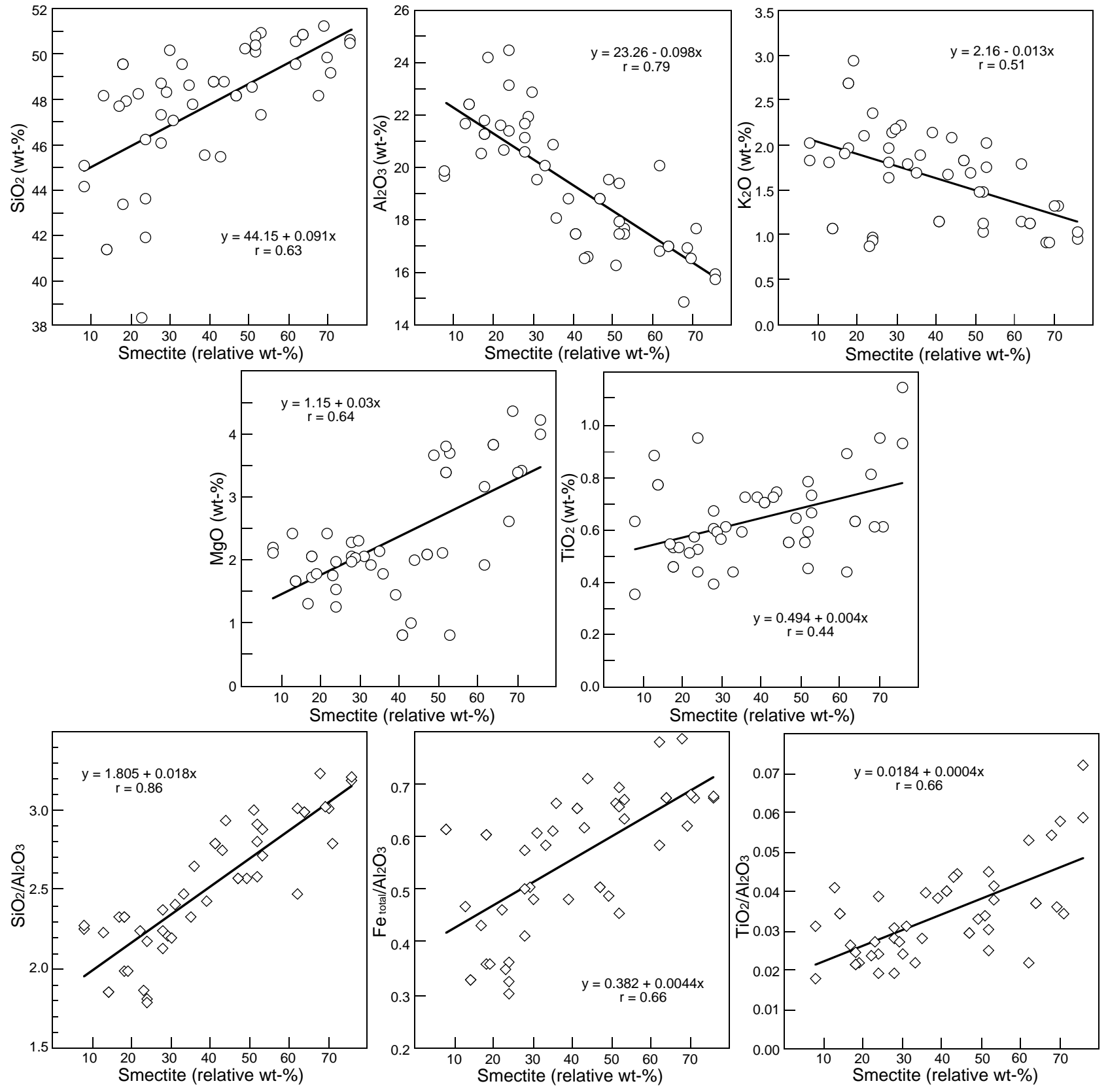

Figure 15. Linear regression plots of $\%$ smectite (from XRD) vs. weight percent oxide and oxide ratios (from SEM/EDS). For a population size equal to 43, correlation coefficients ( $\mathrm{r}$ ) greater than 0.40 indicate that the correlation is statistically significant at the $99.5 \%$ confidence level. Correlations for $\mathrm{Fe}_{\text {total }}(\mathrm{r}=0.35$ ), $\mathrm{CaO}(\mathrm{r}=0.31), \mathrm{K}_{2} \mathrm{O} / \mathrm{Al}_{2} \mathrm{O}_{3}(\mathrm{r}=0.26)$, and $\mathrm{MgO} / \mathrm{Al}_{2} \mathrm{O}_{3}$ have been omitted because they are not statistically significant.

Buatier et al., 1992; Tribble and Yeh, 1994). If this interpretation is correct, then one should expect the diagenetic effects to be most pronounced within stratigraphic or structural intervals that possess the highest permeabilities. In other words, the décollement zone, rather than the underthrust domain, should be the prime suspect for hydrogeochemical acceleration of the illitization reaction.

Many types of data support the contention of enhanced fluid flow near the top of the décollement. The décollement seismic reflector, for example, displays a negative impedance contrast and highly variable amplitude; this seismic response has been attributed to overpressured conditions and fault zone dilation (Shipley et al., 1994). Interpretations of logging-while-drilling (LWD) data also indicate that fluid pressures increase to their highest values within the upper part of the décollement zone (J.C. Moore et al., 1995). Geochemical data from pore fluids show that the chloride minimum is located at the top of, or immediately above, the décollement zone at Sites 671 and 948 (Gieskes et al., 1990a, b; Shipboard Scientific Party, 1995a). This minimum is approximately equal to an $18 \%$ dilution of normal seawater, and the salinity profile decreases steadily down the décollement zone. The concentration of dissolved potassium reaches a minimum just above the top of the décollement (Shipboard Scientific Party, 1995a), and this decrease could be caused by cation uptake into K-rich smectite, as described by Buatier et al. (1992).

Below the décollement, there is little evidence for fluid advection within the underthrusted section. In fact, pore-water salinities within the underthrusted section range from $2.5 \%$ above average seawater salinity to $4 \%-5 \%$ below seawater (Shipboard Scientific Party, 1995a). In addition, concentrations of dissolved potassium increase 


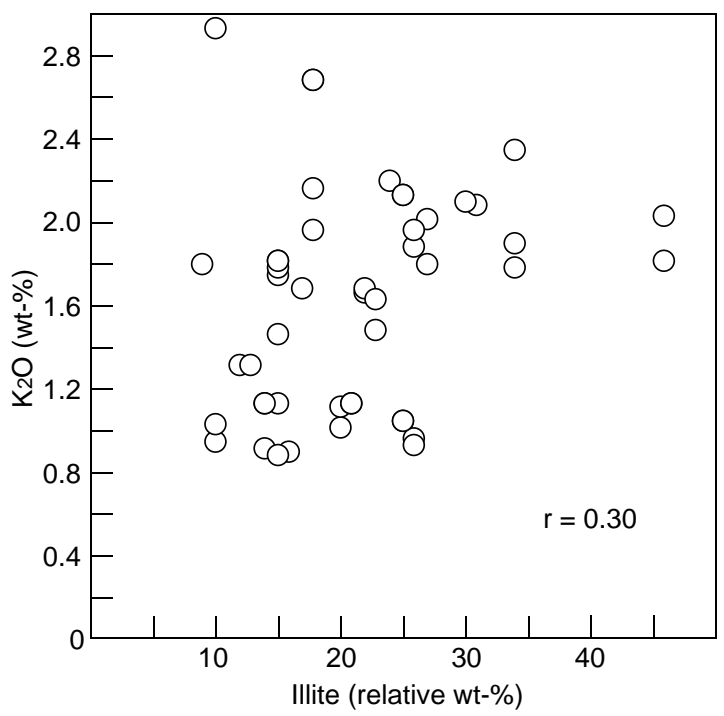

Figure 16. Linear regression plot of $\%$ illite (from XRD) vs. weight percent $\mathrm{K}_{2} \mathrm{O}$ (from SEM/EDS). The r-values for all other correlations except $\mathrm{TiO}_{2} /$ $\mathrm{Al}_{2} \mathrm{O}_{3}$ vs. \% illite $(\mathrm{r}=0.43)$ are less than 0.40 , below the level of statistical significance.

considerably below the base of the décollement, which indicates that large amounts of $\mathrm{K}^{+}$are not being consumed by the mineral solids. This finding is difficult to reconcile with the hypothesis of in situ illitization below the décollement unless the volumetric increase in authigenic illite is too small to effect pore-water chemistry or its effects are masked by another reaction. Perhaps the anomalously coarse-grained Eocene turbidites that are located deeper within the underthrust domain provide additional high-permeability pathways for fluid flow, thereby promoting accelerated diagenesis farther from the prism's toe. This possibility is an important one to consider during refinements of numerical models of prism-wide fluid budgets (e.g., Bekins et al., 1994), but it remains almost entirely speculative. In reality, the potential effects of deep-seated clay-mineral diagenesis still need to be verified by deeper drilling. If a shallow domain of accelerated smectite-to-illite diagenesis really does exist, then it should be restricted to the upper portion of the décollement, where fluid advection is known to be pronounced. Our results fail to substantiate this idea.

\section{CONCLUSIONS}

The relative abundances of smectite within sediments of the northern Barbados subduction zone reach their highest levels in lower to middle Miocene, radiolarian-bearing claystones. The diffuse upper boundary of the décollement zone definitely passes through this stratigraphic interval, but we are not able to identify a unique stratigraphic surface that serves as a marker bed for seaward propagation of the plate-boundary fault. Smectite content decreases gradually down the décollement zone. The sharp base of the décollement coincides with an interval of illite-rich and kaolinite-rich claystone. Clay mineralogy, therefore, appears to control the positions of both maximum and minimum shear strength for sediments as they approach the deformation front.

Although some of the smectite may have formed by in situ alteration of volcanic ash, we conclude that most of the clay minerals are detrital in origin, and that they were transported from multiple sources. Discrete illite within both Units II and III is detrital and was transported from continental sources in South America. Kaolinite proba- bly came from both South American fluvial systems and intense tropical weathering within the Lesser Antilles volcanic arc. Clay-mineral partitioning by size indicates that Unit II claystones contain larger relative abundances of finer-grained smectite, which is consistent with authigenesis of disseminated volcanic ash. On the other hand, we believe that the pyroclastic material was mixed into a background sediment that already contained abundant detrital smectite from South America, as well as detrital smectite from the arc.

The geochemical characteristics of bulk clay-mineral assemblages and smectite separates are highly variable throughout the study area, and this variability supports the contention of multiple detrital sources. The most common type of expandable clay at Sites 948 and 949 appears to be a K-rich, Al-Fe-beidellite. Late Quaternary clay minerals with this type of composition entered the Lesser Antilles forearc and backarc from continental sources in South America. Smectite is also eroded from the Lesser Antilles volcanic arc, but those species are Fe-montmorillonites and Al-Fe-beidellites with high Mg contents and low K contents. Assuming that these geochemical provenance fingerprints are valid for older samples, we conclude that detrital smectites and smectite-rich I/S phases within the prism toe and underthrust domain were derived mostly from South America.

Our study fails to confirm the idea that illitization has been initiated within the underthrust domain near the toe of the Barbados accretionary prism. Acceleration of the smectite-to-illite reaction, if it occurs at unusually low temperatures, should coincide with intervals of enhanced fluid flow. The upper part of the décollement zone represents the most likely site for this phenomenon, but our data fail to verify the expected increases in illite interlayers within I/S mixed layer clays within that zone.

\section{ACKNOWLEDGMENTS}

We thank the crew and technical staff aboard the JOIDES Resolution for their assistance during sample acquisition and shipboard XRD analyses. Funding for shore-based work was provided by the National Science Foundation through the Joint Oceanographic Institutions, U.S. Science Support Program. Sarah Simpson and Terri Zeman assisted with sample preparation, and Lou Ross provided expert technical guidance in SEM/EDS analysis and data processing. We thank Donald Peacor and Martine Buatier for their helpful reviews of the manuscript.

\section{REFERENCES}

Ahn, J.H., and Peacor, D.R., 1986. Transmission and analytical electron microscopy of the smectite-to-illite transition. Clays Clay Miner., 34:165-179.

Baldwin, S.L., Harrison, T.M., and Burke, K., 1986. Fission track evidence for the source of accreted sandstones, Barbados. Tectonics, 5:457-468.

Beaven, P.J., and Dumbleton, M.J., 1966. Clay minerals and geomorphology in four Caribbean islands. Clay Miner., 6:371-382.

Bekins, B.A., and Dreiss, S.J., 1992. A simplified analysis of parameters controlling dewatering in accretionary prisms. Earth Planet. Sci. Lett., 109:275-287.

Bekins, B., McCaffrey, A.M., and Dreiss, S.J., 1994. Influence of kinetics on the smectite to illite transition in the Barbados accretionary prism. $J$. Geophys. Res., 99:18,147-18,158.

Belderson, R.H., Kenyon, N.H., Stride, A.H., and Pelton, C.D., 1984. A "braided" distributary system on the Orinoco deep-sea fan. Mar. Geol., 56:195-206.

Bethke, C.M., and Altaner, S.P., 1986. Layer-by-layer mechanism of smectite illitization and application to a new rate law. Clays Clay Miner., 34:136145.

Biju-Duval, B., Le Quellec, P., Mascle, A., Renard, V., and Valery, P., 1982. Multibeam bathymetric survey and high resolution seismic investigations on the Barbados Ridge complex (eastern Caribbean): a key to the knowl- 
edge and interpretation of an accretionary wedge. Tectonophysics, 86:275-304.

Biju-Duval, B., Moore, J.C., et al., 1984. Init. Repts. DSDP, 78A: Washington (U.S. Govt. Printing Office).

Biscaye, P.E., 1965. Mineralogy and sedimentation of recent deep-sea clays in the Atlantic Ocean and adjacent seas and oceans. Geol. Soc. Amer. Bull., 76:803-832.

Bouysse, P., Westercamp, D., and Andreieff, P., 1990. The Lesser Antilles island arc. In Moore, J.C., Mascle, A., et al., Proc. ODP, Sci. Results, 110: College Station, TX (Ocean Drilling Program), 29-44.

Bruce, C.H., 1984. Smectite dehydration-its relation to structural development and hydrocarbon accumulation in northern Gulf of Mexico basin. AAPG Bull., 68:673-683.

Buatier, M.D., Peacor, D.R., and O'Neil, J.R., 1992. Smectite-illite transition in Barbados accretionary wedge sediments: TEM and AEM evidence for dissolution/crystallization at low temperature. Clays Clay Miner., 40:6580.

Burst, J.F., 1969. Diagenesis of Gulf Coast clayey sediments and its possible relation to petroleum migration. AAPG Bull., 53:73-93.

Capet, X., Chamley, H., Beck, C., and Holtzapffel, T., 1990. Clay mineralogy of Sites 671 and 672, Barbados Ridge accretionary complex and Atlantic abyssal plain: paleoenvironmental and diagenetic implications. In Moore, J.C., Mascle, A., et al., Proc. ODP, Sci. Results, 110: College Station, TX (Ocean Drilling Program), 85-96.

Chamley, H., 1989. Clay Sedimentology: Berlin (Springer-Verlag).

Cleary, W.J., Curran, H.A., and Thayer, P.A., 1984. Barbados Ridge: inner trench slope sedimentation. J. Sediment. Petrol., 54:527-540.

Colten-Bradley, V.A., 1987. Role of pressure in smectite dehydration effects on geopressure and smectite-to-illite transformation. AAPG Bull., 71:1414-1427.

Damuth, J.E., 1977. Late Quaternary sedimentation in the western equatorial Atlantic. Geol. Soc. Am. Bull., 88:695-710.

Dolan, J.F., Beck, C., Ogawa, Y., and Klaus, A., 1990. Eocene-Oligocene sedimentation in the Tiburon Rise/ODP Leg 110 area; an example of significant upslope flow of distal turbidity currents. In Moore, J.C., Mascle, A., et al., Proc. ODP, Sci. Results, 110: College Station, TX (Ocean Drilling Program), 47-83.

Dunoyer de Segonzac, G., 1970. The transformation of clay minerals during diagenesis and low-grade metamorphism: a review. Sedimentology, $15: 281-346$.

Eberl, D., and Hower, J., 1976. Kinetics of illite formation. Geol. Soc. Am. Bull., 87:1326-1330.

Faugères, J.C., Gonthier, E., Griboulard, R., and Masse, L., 1993. Quaternary sandy deposits and canyons on the Venezuelan margin and south Barbados accretionary prism. Mar. Geol., 110:115-142.

Faugères, J.C., Gonthier, E., Masse, L., Parra, M., Pons, J.C., and Pujol, C., 1991. Quaternary deposits on the South Barbados accretionary prism. Mar. Geol., 96:247-267.

Ferguson, I.J., Westbrook, G.K., Langseth, M.G., and Thomas, G.P., 1993. Heat flow and thermal models of the Barbados Ridge accretionary complex. J. Geophys. Res., 984121-4142.

Fisher, A.T., and Hounslow, M.W., 1990. Transient fluid flow through the toe of the Barbados accretionary complex: constraints from Ocean Drilling Program Leg 110 heat flow studies and simple models. J. Geophys. Res., 95:8845-8858.

Fisher, A.T., and Underwood, M.B., 1995. Calibration of an X-ray diffraction method to determine relative mineral abundances in bulk powders using matrix singular value decomposition: a test from the Barbados accretionary complex. In Shipley, T.H., Ogawa, Y., Blum, P., et al., Proc. ODP, Init. Repts., 156: College Station, TX (Ocean Drilling Program), 29-37.

Foucher, J.-P., Le Pichon, X., Lallemant, S., Hobart, M.A., Henry, P., Benedetti, M., Westbrook, G.K., and Langseth, M.G., 1990. Heat flow, tectonics, and fluid circulation at the toe of the Barbados Ridge accretionary prism. J. Geophys. Res., 95:8859-8868.

Freed, R.L., and Peacor, D.R., 1989a. Geopressured shale and sealing effect of smectite to illite transition. AAPG Bull., 73:1223-1232.

, 1989b. Variability in temperature of the smectite/illite reaction in Gulf Coast sediments. Clay Miner., 24:171-180.

Gandais, V., 1987. Clay mineral sources of the Grenada Basin, southeastern Caribbean. Clay Miner., 22:395-400.

Gieskes, J.M., Blanc, G., Vrolijk, P., Elderfield, H., and Barnes, R., 1990a. Interstitial water chemistry-major constituents. In Moore, J.C., Mascle, A., et al., Proc. ODP, Sci. Results, 110: College Station, TX (Ocean Drilling Program), $155-178$.
Gieskes, J.M., Vrolijk, P., and Blanc, G., 1990b. Hydrogeochemistry of the northern Barbados accretionary complex transect: Ocean Drilling Program Leg 110. J. Geophys. Res., 95:8809-8818

Haq, B.U., Hardenbol, J., and Vail, P.R., 1988. Mesozoic and Cenozoic chronostratigraphy and cycles of sea-level change. In Wilgus, C.K., Hastings, B.S., Kendall, C.G.St.C., Posamentier, H.W., Ross, C.A., and Van Wagoner, J.C. (Eds.), Sea-Level Changes-An Integrated Approach. Spec. Publ.-Soc. Econ. Paleontol. Mineral., 42:72-108.

Hathon, E.G., 1992. X-ray diffraction and transmission electron microscopy study of the surface charge on the illite and smectite components of illite/ smectite mixed-layer clays [Ph.D. dissert.]. Univ. of Missouri, Columbia.

Hathon, E.G., and Underwood, M.B., 1991. Clay mineralogy and chemistry as indicators of hemipelagic sediment dispersal south of the Aleutian arc. Mar. Geol., 97:145-166.

Hodder, A.P.W., Naish, T.R., and Nelson, C.S., 1993. A two-stage model for the formation of smectite from detrital volcanic glass under shallowmarine conditions. Mar. Geol., 109:279-285.

Hoffman, J., and Hower, J., 1979. Clay mineral assemblages as low grade metamorphic geothermometers: application to the thrust faulted disturbed belt of Montana, U.S.A. Spec. Publ-Soc. Econ. Paleontol. Mineral., 26:55-79.

Housen, B.A., Tobin, H.J., Labaume, P., Leitch, E.C., Maltman, A.J., Shipley, T., Ogawa, Y., Ashi, J., Blum, P., Brückmann, W., Felice, F., Fisher, A., Goldberg, D., Henry, P., Jurado, M.-J., Kastner, M., Laier, T., Meyer, A., Moore, J.C., Moore, G., Peacock, S., Rabaute, A., Steiger, T., Underwood, M., Xu, Y., Yin, H., Zheng, Y., and Zwart, G., 1996. Strain decoupling across the décollement of the Barbados accretionary prism. Geology, 24:127-130.

Hower, J., Eslinger, E.V., Hower, M.E., and Perry, E.A., 1976. Mechanism of burial metamorphism of argillaceous sediment. 1. Mineralogical and chemical evidence. Geol. Soc. Am. Bull., 87:725-737.

Huang, W-L., Longo, J.M., and Pevear, D.R., 1993. An experimentally derived kinetic model for smectite-to-illite conversion and its use as a geothermometer. Clays Clay Miner., 41:162-177.

Inoue, A., Bouchet, A., Velde, B., and Meunier, A., 1989. Convenient technique for estimating smectite layer percentage in randomly interstratified illite/smectite minerals. Clays Clay Miner., 37:227-234.

Kagami, H., 1985. Internal structures of the accretionary wedge in the Nankai Trough off Shikoku, southwestern Japan. In Nasu, N., Kobayashi, K., Uyeda, S., Kushiro, I., and Kagami, H. (Eds.), Formation of Active Ocean Margins: Tokyo (Terra Sci. Publ.), 193-219.

Kasper, D.C., and Larue, D.K., 1986. Paleogeographic and tectonic implications of quartzose sandstones of Barbados. Tectonics, 6:837-854.

Lallemant, S.J.C., Henry, P., Le Pichon, X., and Foucher, J.P., 1990. Detailed structure and possible fluid paths at the toe of the Barbados accretionary wedge (ODP Leg 110 area). Geology, 18:854-857.

Langseth, M.G., Westbrook, G.K., and Hobart, M., 1990. Contrasting geothermal regimes of the Barbados Ridge accretionary complex. J. Geophys. Res., 95:8829-8843.

Latouche, C., and Maillet, N., 1984. Evolution of Cenozoic clay assemblages in the Barbados Ridge (Deep Sea Drilling Project Sites 541, 542, 543). In Biju-Duval, B., Moore, J.C., et al., Init. Repts. DSDP, 78A: Washington (U.S. Govt. Printing Office), 343-356.

Mascle, A., Moore, J.C., et al., 1988. Proc. ODP, Init. Repts. (Pt. A), 110: College Station, TX (Ocean Drilling Program).

Massé, L., Faugères, J.C., and Gonthier, E., 1995. Quaternary deposits and sediment fluxes at the toe of the Barbados accretionary prism. Geo-Mar. Lett., 15:99-105.

McManus, D.A., 1991. Suggestions for authors whose manuscripts include quantitative clay mineral analysis by X-ray diffraction. Mar. Geol., 98:1-5.

Moore, D.M., and Reynolds, R.C., Jr., 1989. X-ray Diffraction and the Identification and Analysis of Clay Minerals: Oxford (Oxford Univ. Press).

Moore, G.F., Zhao, Z., Shipley, T.H., Bangs, N., and Moore, J.C., 1995. Structural setting of the Leg 156 area, northern Barbados Ridge accretionary prism. In Shipley, T.H., Ogawa, Y., Blum, P., et al., Proc. ODP, Init. Repts., 156: College Station, TX (Ocean Drilling Program), 13-27.

Moore, J.C., Biju-Duval, B., Bergen, J.A., Blackington, G., Claypool G.E., Cowan, D.S., Duennebier, F., Guerra, R.T., Hemleben, C.H.J., Hussong, D., Marlow, M.S., Natland, J.H., Pudsey, C.J., Renz, G.W., Tardy, M., Willis, M.E., Wilson, D., and Wright, A.A., 1982. Offscraping and underthrusting of sediment at the deformation front of the Barbados Ridge: Deep Sea Drilling Project Leg 78A. Geol. Soc. Am. Bull., 93:1065-1077.

Moore, J.C., Mascle, A., Taylor, E., Andreieff, P., Alvarez, F., Barnes, R., Beck, C., Behrmann, J., Blanc, G., Brown, K., Clark, M., Dolan, J., 
Fisher, A., Gieskes, J., Hounslow, M., McLellan, P., Moran, K., Ogawa, Y., Sakai, T., Schoonmaker, J., Vrolijk, P., Wilkens, R., and Williams, C., 1988. Tectonics and hydrogeology of the northern Barbados Ridge: Results from Ocean Drilling Program Leg 110. Geol. Soc. Amer. Bull., 100:1578-1593.

Moore, J.C., Mascle, A., et al., 1990. Proc. ODP, Sci. Results, 110: College Station, TX (Ocean Drilling Program).

Moore, J.C., Shipley, T.H., Goldberg, D., Ogawa, Y., Filice, F., Fisher, A., Jurado, M.-J., Moore, G.F., Rabaute, A., Yin, H., Zwart, G., Brückmann, W., Henry, P., Ashi, J., Blum, P., Meyer, A., Housen, B., Kastner, M., Labaume, P., Laier, T., Leitch, E.C., Maltman, A.J., Peacock, S., Steiger, T.H., Tobin, H.J., Underwood, M.B., Xu, Y., and Zheng, Y., 1995. Abnormal fluid pressures and fault zone dilation in the Barbados accretionary prism: evidence from logging while drilling. Geology, 23:605-608.

Moore, J.C., and Vrolijk, P., 1992. Fluids in accretionary prisms. Rev. Geophys., 30:113-135.

Naish, T.R., Nelson, C.S., and Hodder, A.P.W., 1993. Evolution of Holocene sedimentary bentonite in a shallow-marine embayment, Firth of Thames, New Zealand. Mar. Geol., 109:267-278.

Parra, M., Pons, J.C., and Ferragne, A., 1986. Two potential sources for Holocene clay sedimentation in the Caribbean Basin: the Lesser Antilles arc and the South American continent. Mar. Geol., 72:287-304.

Perry, E., and Hower, J., 1970. Burial diagenesis in Gulf Coast pelitic sediments. Clays Clay Miner., 18:165-177.

Pudsey, C.J., 1984. X-ray mineralogy of Miocene and older sediments from Deep Sea Drilling Project Leg 78A. In Biju-Duval, B., Moore, J.C., et al., Init. Repts. DSDP, 78A: Washington (U.S. Govt. Printing Office), 325342.

Reid, R.P., Carey, S.N., Staerker, T.S., and Ask, M., 1994. Sedimentology of the collision zone between the North d'Entrecasteaux Ridge and New Hebrides Island Arc (Sites 827, 828, and 829). In Greene, H.G., Collot, J.-Y., Stokking, L.B., et al., Proc. ODP, Sci. Results, 134: College Station, TX (Ocean Drilling Program), 73-88.

Reid, R.P., Carey, S.N., and Ross, D.R., 1996. Late Quaternary sedimentation in the Lesser Antilles island arc. Geol. Soc. Am. Bull., 108:78-100.

Reynolds, R.C., Jr., and Hower, J., 1970. The nature of interlayering in mixed-layer illite-montmorillonites. Clays Clay Miner., 18:25-36.

Ross, L.M., Zhao, N., and Johns, W.D., 1993. Quantitative X-ray microanalysis of oriented clay minerals: an SEM/EDS technique to determine major element chemistry. Proc. Clay Miner. Soc., 8.

Schoonmaker, J., 1986. Clay mineralogy and diagenesis of sediments from deformation zones in the Barbados accretionary wedge (DSDP Leg 78A). In Moore, J.C. (Ed.), Structural Fabrics in Deep Sea Drilling Project Cores from Forearcs. Mem.-Geol. Soc. Am., 166: 105-116.

Shipboard Scientific Party, 1988a. Site 671. In Mascle, A., Moore, J.C., et al., Proc. ODP, Init. Repts (Pt. A)., 110: College Station, TX (Ocean Drilling Program), 67-204.

- 1988b. Site 672. In Mascle, A., Moore, J.C., et al., Proc. ODP, Init. Repts (Pt. A)., 110: College Station, TX (Ocean Drilling Program), 205-310.

, 1995a. Site 948. In Shipley, T.H., Ogawa, Y., Blum, P., et al., Proc. ODP, Init. Repts., 156: College Station, TX (Ocean Drilling Program), 87-192.

1995b. Site 949. In Shipley, T.H., Ogawa, Y., Blum, P., et al., Proc. ODP, Init. Repts., 156: College Station, TX (Ocean Drilling Program), 193-257.

Shipley, T.H., Ogawa, Y., Blum, P., et al., 1995. Proc. ODP, Init. Repts., 156: College Station, TX (Ocean Drilling Program).

Shipley, T.H., Moore, G.F., Bangs, N.L., Moore, J.C., and Stoffa, P.L., 1994. Seismically inferred dilatancy distribution, northern Barbados Ridge décollement: implications for fluid migration and fault strength. Geology, 22:411-414.
Sigurdsson, H., Sparks, R.S.J., Carey, S.N., and Huang, T.C., 1980. Volcanogenic sedimentation in the Lesser Antilles arc. J. Geol., 88:523-540.

Srodon, J., 1980. Precise identification of illite/smectite interstratification by X-ray powder diffraction. Clays Clay Miner., 28:401-411.

1981. X-ray identification of randomly interstratified illite-smectites in mixtures with discrete illite. Clay Miner., 16:297-304.

Stride, A.H., Belderson, R.H., and Kenyon, N.H., 1982. Structural grain, mud volcanoes and other features on the Barbados Ridge complex revealed by GLORIA long-range side-scan sonar. Mar. Geol., 49:187196.

Tomita, K., Takahashi, H., and Watanabe, T., 1988. Quantification curves for mica/smectite interstratifications by X-ray powder diffraction. Clays Clay Miner., 36:258-262.

Tribble, J.S., 1990. Clay diagenesis in the Barbados accretionary complex: potential impact on hydrology and subduction dynamics. In Moore, J.C., Mascle, A., et al., Proc. ODP, Sci. Results, 110: College Station, TX (Ocean Drilling Program), 97-110.

Tribble, J.S., and Yeh, H-W., 1994. Origin of smectite and illite-smectite in the Barbados accretionary complex: Oxygen isotopic evidence. Geology, 22:219-222.

Underwood, M.B., Orr, R., Pickering, K., and Taira, A., 1993a. Provenance and dispersal patterns of sediments in the turbidite wedge of Nankai Trough. In Hill, I.A., Taira, A., Firth, J.V., et al., Proc. ODP, Sci. Results, 131: College Station, TX (Ocean Drilling Program), 15-34.

Underwood, M.B., Pickering, K., Gieskes, J.M., Kastner, M., and Orr, R., 1993b. Sediment geochemistry, clay mineralogy, and diagenesis: a synthesis of data from Leg 131, Nankai Trough. In Hill, I.A., Taira, A., Firth, J.V., et al., Proc. ODP, Sci. Results, 131: College Station, TX (Ocean Drilling Program), 343-363.

Velde, B., and Vasseur, G., 1992. Estimation of the diagenetic smectite to illite transformation in time-temperature space. Am. Mineral., 77:967976.

Vrolijk, P., 1990. On the mechanical role of smectite in subduction zones. Geology, 18:703-707.

Vrolijk, P., Fisher, A., and Gieskes, J., 1991. Geochemical and geothermal evidence for fluid migration in the Barbados accretionary prism (ODP Leg 110). Geophys. Res. Lett., 18:947-950.

Weir, A.H., Ormerod, E.C., and El Mansey, M.I.I., 1975. Clay mineralogy of sediments of the western Nile Delta. Clay Miner, 10:369-386.

Westbrook, G.K., 1982. The Barbados Ridge Complex: tectonics of a mature forearc system. In Leggett, J.K. (Ed.), Trench and Forearc Geology. Geol. Soc. Spec. Publ. London, 10: 275-290.

Westbrook, G.K., Ladd, J.W., Buhl, P., Bangs, N., and Tiley, G.J., 1988. Cross section of an accretionary wedge: Barbados Ridge complex. Geology, 16:631-635.

Westbrook, G.K., Mascle, A., and Biju-Duval, B., 1984. Geophysics and structure of the Lesser Antilles forearc. In Biju-Duval, B., Moore, J.C., et al., Init. Repts. DSDP, 78A: Washington (U.S. Govt. Printing Office), 2338.

Wilkens, R., McLellan, P., Moran, K., Tribble, J.S., Taylor, E., and Verduzco, E., 1990. Diagenesis and dewatering of clay-rich sediments, Barbados accretionary prism. In Moore, J.C., Mascle, A., et al., Proc. ODP, Sci. Results, 110: College Station, TX (Ocean Drilling Program), 309-320.

Wright, A., 1984. Sediment distribution and depositional processes operating in the Lesser Antilles Intraoceanic Island Arc, eastern Caribbean. In BijuDuval, B., Moore, J.C., et al., Init. Repts. DSDP, 78A: Washington (U.S. Govt. Printing Office), 301-324.

Date of initial receipt: 31 January 1996

Date of acceptance: 1 October 1996

Ms 156SR-001 

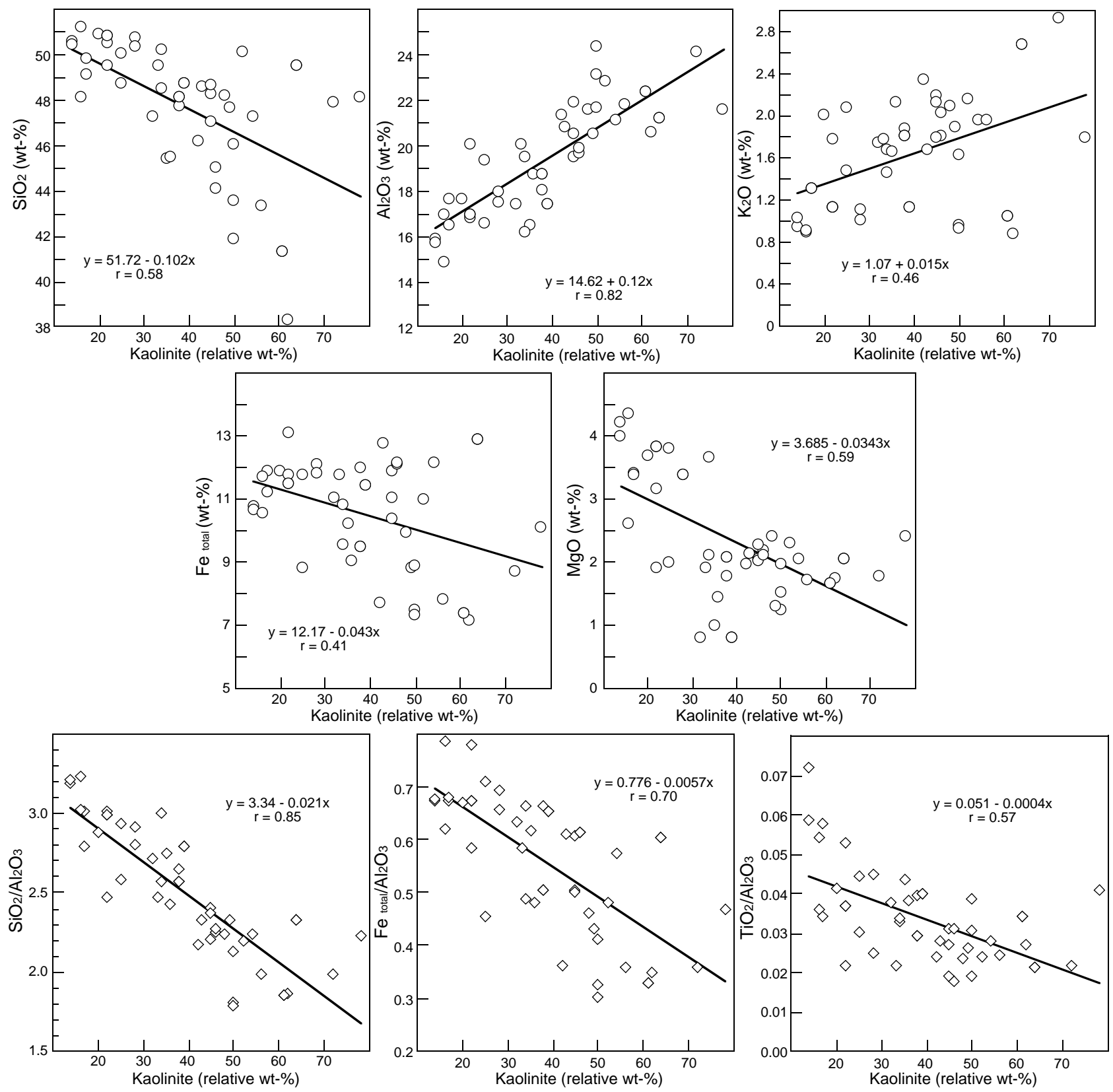

Figure 17. Linear regression plots of \% kaolinite (from XRD) vs. weight percent oxide and oxide ratios (from SEM/EDS). Regression results for \% kaolinite vs. $\mathrm{TiO}_{2}, \mathrm{~K}_{2} \mathrm{O} / \mathrm{Al}_{2} \mathrm{O}_{3}, \mathrm{MgO} / \mathrm{Al}_{2} \mathrm{O}_{3}$, and $\mathrm{CaO} / \mathrm{Al}_{2} \mathrm{O}_{3}$ have been omitted because their $\mathrm{r}$-values are less than 0.40 . 